\title{
Improved MSSM Higgs mass calculation using the 3-loop FlexibleEFTHiggs approach including $x_{t}$-resummation
}

\author{
Thomas Kwasnitza, ${ }^{a}$ Dominik Stöckinger ${ }^{a}$ and Alexander Voigt ${ }^{b}$ \\ ${ }^{a}$ Institut für Kern- und Teilchenphysik, Technische Universität Dresden, \\ Zellescher Weg 19, 01069 Dresden, Germany \\ ${ }^{b}$ Institut fr̈ mathematische, naturwissenschaftliche und technische Bildung, \\ Europa-Universität Flensburg, \\ Auf dem Campus 1, 24943 Flensburg, Germany \\ E-mail: thomas.kwasnitza@mailbox.tu-dresden.de, \\ dominik.stoeckinger@tu-dresden.de, alexander.voigt@uni-flensburg.de
}

ABSTRACT: We present an improved calculation of the light CP-even Higgs boson pole mass in the MSSM based on the FlexibleEFTHiggs hybrid method. The calculation resums large logarithms to all orders and includes power-suppressed terms at fixed order. It uses stateof-the-art 2- and 3-loop matching of the quartic Higgs coupling and renormalization group running up to 4-loop, resulting in a resummation of large logarithmic corrections up to $\mathrm{N}^{3} \mathrm{LL}$ level. A conceptually novel ingredient is the expansion of the matching conditions in terms of high-scale MSSM parameters instead of SM parameters. In this way leading QCD-enhanced terms in the stop-mixing parameter are effectively resummed, leading to an improved numerical convergence of the perturbative expansion. Furthermore, the avoidance of double counting of loop corrections is more transparent than in other approaches and more independent of the high-scale model. We present numerical results and a detailed discussion of theoretical uncertainties for standard benchmark scenarios.

KEYWORds: Supersymmetry Phenomenology

ARXiv EPRINT: 2003.04639 


\section{Contents}

1 Introduction $\quad 2$

2 Definition of the Standard Model and the MSSM 4

3 Matching procedure in general $\quad 5$

$\begin{array}{lll}3.1 \text { Basics of the effective field theory approach } & 6\end{array}$

$\begin{array}{lll}3.2 & \text { Parametrization of the matching relations } & 7\end{array}$

3.3 Matching of the quartic Higgs coupling 8

4 New FlexibleEFTHiggs matching procedure 10

$\begin{array}{lll}4.1 & \text { FlexibleEFTHiggs matching conditions } & 10\end{array}$

$\begin{array}{lll}4.2 & \text { Perturbative expansion of the matching conditions } & 12\end{array}$

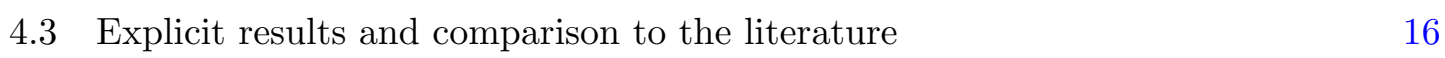

$5 \quad$ Resummation of leading squark mixing contributions $\quad 22$

6 Running and matching procedure at the electroweak scale 26

$\begin{array}{lll}6.1 & \text { Running to the electroweak scale } & 27\end{array}$

6.2 Matching of SM couplings to observables 27

7 Numerical results $\quad \mathbf{3 0}$

7.1 Impact of higher orders, the new parametrization and the $x_{t}$-resummation $\quad 30$

$\begin{array}{lll}7.2 & \text { Comparison to state-of-the-art calculations } 33\end{array}$

7.3 Further details on the comparison of hybrid and pure EFT calculations $\quad 37$

8 Uncertainty estimation $\quad 40$

$\begin{array}{lll}8.1 & \text { High-scale uncertainty } & 40\end{array}$

$\begin{array}{lll}8.2 & \text { Low-scale uncertainty } & 44\end{array}$

8.3 Numerical size of individual uncertainties 44

$\begin{array}{lll}\text { 8.3.1 High-scale uncertainty } & 44\end{array}$

$\begin{array}{lll}\text { 8.3.2 } & \text { Low-scale uncertainty } & 47\end{array}$

$\begin{array}{ll}8.4 \text { Combined uncertainty } & 48\end{array}$

9 Conclusions $\quad 50$

A Higgs pole mass matching for $\Delta \lambda$ at $\mathcal{O}\left(y_{t}^{6}\right)$ 


\section{Introduction}

Since the discovery of the Higgs boson [1, 2], the Higgs boson mass $M_{h}=(125.10 \pm$ $0.14) \mathrm{GeV}[3,4]$ has become a high-precision observable [5], which represents another useful tool to search for physics beyond the Standard Model (SM) and constrain the large zoo of proposed SM extensions, such as supersymmetric (SUSY) models. The latter are particularly interesting, as they require the existence of scalar fields and predict the quartic Higgs coupling and thus the Higgs boson mass. The precise prediction of the SM-like Higgs boson mass in the Minimal Supersymmetric Standard Model (MSSM), however, is a long-standing challenge, because in viable MSSM scenarios large radiative loop corrections of the order $\Delta m_{h}^{2} \sim(100 \mathrm{GeV})^{2}$ are required, resulting in a large truncation error of the perturbation series.

There are two main mechanisms which can generate such large loop corrections: $(i)$ Large SUSY masses $M_{S}$ (in particular stop masses) lead to large logarithmic corrections of the form $\log \left(M_{S} / v\right)$, where $v$ represents the electroweak scale. (ii) A large mixing in the stop sector, governed by the parameter $X_{t}$, leads to power corrections of the order $\left(X_{t} / M_{S}\right)^{n}$. Effective field theory (EFT) techniques are a well-known tool to perform a resummation of the large logarithmic corrections, thus effectively avoiding a large truncation error of the contributions from mechanism $(i)$. Concerning the $\left(X_{t} / M_{S}\right)^{n}$ power corrections, however, no similar resummation technique has been used so far. In the present work we present a technique to effectively resum leading terms in $X_{t}$ in the prediction of the light Higgs boson mass.

There are different approaches to calculate the Higgs boson mass in supersymmetric models, which can be roughly classified into fixed-order [6-38], ${ }^{1}$ EFT [40-51], and hybrid [52-61] approaches, which combine the virtues of the former two. Fixed-order approaches truncate the perturbation series at a certain order in loops and couplings, neglecting in particular large logarithmic corrections arising at higher orders. Thus, when $M_{S} \gg v$, the fixed-order approaches usually suffer from a large uncertainty due to missing large higher-order corrections. EFT approaches, on the other hand, resum the large logarithmic corrections to all orders, but usually neglect terms of the order $v^{2} / M_{S}^{2}$. As a consequence, EFT approaches become imprecise when $M_{S} \sim v$.

Hybrid approaches combine the virtues of fixed-order and EFT calculations: they resum large logarithmic corrections to all orders and include terms suppressed by $v^{2} / M_{S}^{2}$ at fixed order. A first variant of such a hybrid approach was presented in ref. [52] and implemented into FeynHiggs. This approach uses a "subtraction method", where the large logarithmic corrections are subtracted from a fixed-order calculation and are replaced by resummed logarithms, avoiding double counting. This method was refined in refs. [53, 57] and applied in the context of the $\overline{\mathrm{DR}}^{\prime}$ scheme in ref. [60].

An alternative way to realize a hybrid approach was presented in refs. $[54,56]$. This so-called FlexibleEFTHiggs approach is an EFT calculation in which the matching condition is suitably modified such that terms suppressed by powers of $v^{2} / M_{S}^{2}$ are included in the quartic Higgs coupling. One advantage of this method is the structural simplicity

\footnotetext{
${ }^{1}$ Here we focus on multi-loop calculations. For further references see the review [39] and the references therein.
} 
of the matching condition. As a result, the method is well suited for automation and has thus been implemented into the generic spectrum generators FlexibleSUSY [56, 62] and SARAH/SPheno [55]. A difficulty of the FlexibleEFTHiggs approach is to make sure that large logarithms cancel in the matching between the EFT and the UV model, as required. Indeed, avoiding double counting leads to significant complications in all hybrid calculations $[56,57,63]$.

In this paper we present an extension of the FlexibleEFTHiggs hybrid approach with a matching of the quartic Higgs coupling $\hat{\lambda}$ beyond 1-loop level (next-to-leading order, NLO) and apply it to perform a state-of-the-art hybrid calculation of the light CP-even Higgs boson mass in the real MSSM. Thereby our calculation incorporates several conceptual changes and significant improvements:

- We parametrize the matching calculation at the high-energy scale in terms of parameters of the UV model (i.e. the MSSM). This is in contrast to the usually chosen parametrization in terms of EFT parameters. Our "full-model parametrization" has several significant advantages. An important advantage is that the cancellation of large logarithmic corrections in the matching is more transparent. Furthermore, our parametrization allows for a computer algebraic implementation which is to a large extent independent of the chosen UV model. This fact enables the straightforward application to a large class of SUSY models. The detailed discussion of the different possible parametrizations is presented in section 3 .

- In our application to the MSSM we include the state-of-the-art radiative corrections in the matching up to the 3-loop level at $\mathcal{O}\left(1 \ell+g_{3}^{2}\left(y_{t}^{4}+y_{b}^{4}\right)+\left(y_{t}^{2}+y_{b}^{2}\right)^{3}+\left(y_{t}^{2}+y_{\tau}^{2}\right)^{3}+g_{3}^{4} y_{t}^{4}\right)$ and perform renormalization-group running up to 4-loop level in QCD. As a result, our calculation reaches a precision of $\mathrm{N}^{3} \mathrm{LO}$ with a resummation of $\mathrm{N}^{3} \mathrm{LL}$, comparable with the calculation presented in ref. [60]. The details of the matching of the MSSM to the SM are presented in section 4 , and numerical results are shown in sections 7-8.

- The most important advantage of our new approach and the chosen full-model parametrization is the effective resummation of QCD-enhanced terms leading in the stop mixing parameter $X_{t}$, which is presented in section 5 . More specifically, we show that the highest power contributions at $\mathcal{O}\left(y_{t}^{4} g_{3}^{2 n}, y_{t}^{2} g_{1,2}^{2} g_{3}^{2 n}\right)$ for all $n>0$ are captured by our procedure. As a result, the perturbation expansion of the Higgs boson mass in terms of the MSSM parameters stabilizes significantly for large $X_{t}$, leading to a reduced theory uncertainty of the prediction.

We begin with a recap of the SM and the MSSM in section 2, introducing our conventions. section 3 gives a general overview of the implementation of the EFT approach, discussing in particular the role of the parametrization. Our new realization of the FlexibleEFTHiggs approach within a numerical code is discussed in section 4 . In section 5 we show how our chosen parametrization in terms of MSSM parameters results in a resummation of highest power $X_{t}$ contributions as described above. After a study of the numerical results of our new calculation in section 7 , we perform a thorough analysis of the remaining theory uncertainty of our calculation in section 8 . 


\section{Definition of the Standard Model and the MSSM}

In the following we will denote the Standard Model (SM) parameters, defined in the $\overline{\mathrm{MS}}$ scheme, as

$$
\hat{P}=\left\{\hat{g}_{1}, \hat{g}_{2}, \hat{g}_{3}, \hat{y}_{t}, \hat{y}_{b}, \hat{y}_{\tau}, \hat{\lambda}, \hat{v}\right\}
$$

where $\hat{g}_{1}=\sqrt{5 / 3} \hat{g}_{Y}$ and $\hat{g}_{Y}, \hat{g}_{2}$ and $\hat{g}_{3}$ denote the gauge couplings of the gauge groups $\mathrm{U}(1)_{Y}, \mathrm{SU}(2)_{L}$ and $\mathrm{SU}(3)_{C}$, respectively. The Yukawa couplings of the top quark, bottom quark and tau lepton are denoted as $\hat{y}_{t}, \hat{y}_{b}$ and $\hat{y}_{\tau}$, respectively. The $1^{\text {st }}$ and $2^{\text {nd }}$ generation Yukawa couplings as well as CP-violation effects are neglected and we will set the CKM and PMNS matrices to unity. The quartic coupling $\hat{\lambda}$ of the SM Higgs field $\Phi$ is defined by the Higgs potential

$$
V(\Phi)=\hat{\mu}^{2}|\Phi|^{2}+\frac{\hat{\lambda}}{2}|\Phi|^{4} .
$$

We decompose the Higgs field as

$$
\Phi=\left(\begin{array}{c}
G^{+} \\
\frac{1}{\sqrt{2}}\left(\hat{v}+h+i G^{0}\right)
\end{array}\right),
$$

where $h$ is the SM Higgs particle, $\hat{v} \equiv \sqrt{2}\langle\Phi\rangle$ is the Higgs vacuum expectation value (VEV) (i.e. the minimum of the SM effective potential) which satisfies $\hat{v}=\left(-2 \hat{\mu}^{2} / \hat{\lambda}\right)^{1 / 2} \approx 246 \mathrm{GeV}$ at tree level and $G^{0, \pm}$ are the SM Goldstone bosons. After spontaneous electroweak symmetry breaking, the $\overline{\mathrm{MS}}$ masses for the top, bottom and tau fermion and for the heavy physical bosons are given by

$$
\begin{aligned}
& \hat{m}_{t}=\frac{\hat{y}_{t} \hat{v}}{\sqrt{2}}, \quad \hat{m}_{b}=\frac{\hat{y}_{b} \hat{v}}{\sqrt{2}}, \quad \hat{m}_{\tau}=\frac{\hat{y}_{\tau} \hat{v}}{\sqrt{2}}, \\
& \hat{m}_{W}=\frac{\hat{g}_{2} \hat{v}}{2}, \quad \hat{m}_{Z}=\frac{\hat{v}}{2} \sqrt{\hat{g}_{Y}^{2}+\hat{g}_{2}^{2}}, \quad \hat{m}_{h}^{2}=\hat{\lambda} \hat{v}^{2} .
\end{aligned}
$$

For convenience we define in addition the following symbols:

$$
\hat{\alpha}_{t}=\frac{\hat{y}_{t}^{2}}{4 \pi}, \quad \hat{\alpha}_{s}=\frac{\hat{g}_{3}^{2}}{4 \pi}, \quad \hat{\alpha}_{\mathrm{em}}=\frac{\hat{e}^{2}}{4 \pi}, \quad \hat{e}=\frac{\hat{g}_{Y} \hat{g}_{2}}{\sqrt{\hat{g}_{Y}^{2}+\hat{g}_{2}^{2}}} .
$$

We denote the corresponding relevant parameters of the ( $R$-parity conserving) Minimal Supersymmetric Standard Model (MSSM), defined in the $\overline{\mathrm{DR}}^{\prime}$ scheme, as

$$
P=\left\{g_{1}, g_{2}, g_{3}, y_{t}, y_{b}, y_{\tau}, v\right\},
$$

where $g_{1}=\sqrt{5 / 3} g_{Y}$ and $v=\left(v_{u}^{2}+v_{d}^{2}\right)^{1 / 2}$, whereas $v_{u}$ and $v_{d}$ denote the VEVs of the up- and down-type Higgs fields which represent the minimum of the effective potential in the MSSM,

$$
\left\langle H_{u}\right\rangle=\frac{1}{\sqrt{2}}\left(\begin{array}{c}
0 \\
v_{u}
\end{array}\right), \quad\left\langle H_{d}\right\rangle=\frac{1}{\sqrt{2}}\left(\begin{array}{c}
v_{d} \\
0
\end{array}\right)
$$


If not stated otherwise, we define $\tan \beta=v_{u} / v_{d}$. After the spontaneous electroweak symmetry breaking in the MSSM, the $\overline{\mathrm{DR}}^{\prime}$ masses for the top, bottom and tau fermion as well as the SM-like Higgs in the decoupling limit are given by

$$
\begin{array}{rlrl}
m_{t} & =\frac{y_{t} v_{u}}{\sqrt{2}}, \quad m_{b}=\frac{y_{b} v_{d}}{\sqrt{2}}, & m_{\tau}=\frac{y_{\tau} v_{d}}{\sqrt{2}}, \\
m_{h}^{2} & =\frac{1}{4}\left(g_{Y}^{2}+g_{2}^{2}\right) v^{2} \cos ^{2}(2 \beta) .
\end{array}
$$

We neglect inter-generation sfermion mixing, so the $\overline{\mathrm{DR}}^{\prime}$ masses of the stops, sbottoms and staus are given by the eigenvalues of the mass matrices

$$
\begin{aligned}
\mathrm{M}_{t} & =\left(\begin{array}{cc}
m_{t}^{2}+m_{\tilde{q}_{3}}^{2} & m_{t} X_{t} \\
m_{t} X_{t} & m_{t}^{2}+m_{\tilde{u}_{3}}^{2}
\end{array}\right), \\
\mathrm{M}_{b} & =\left(\begin{array}{cc}
m_{b}^{2}+m_{\tilde{q}_{3}}^{2} & m_{b} X_{b} \\
m_{b} X_{b} & m_{b}^{2}+m_{\tilde{d}_{3}}^{2}
\end{array}\right), \\
\mathrm{M}_{\tau} & =\left(\begin{array}{cc}
m_{\tau}^{2}+m_{\tilde{l}_{3}}^{2} & m_{\tau} X_{\tau} \\
m_{\tau} X_{\tau} & m_{\tau}^{2}+m_{\tilde{e}_{3}}^{2}
\end{array}\right),
\end{aligned}
$$

where $m_{\tilde{q}_{3}}^{2}, m_{\tilde{u}_{3}}^{2}, m_{\tilde{d}_{3}}^{2}, m_{\tilde{l}_{3}}^{2}$ and $m_{\tilde{e}_{3}}^{2}$ denote the squared soft-breaking mass parameters of the left- and right-handed $3^{\text {rd }}$ generation squarks and sleptons and electroweak contributions from $D$-terms have been omitted. ${ }^{2}$ The sfermion mixing parameters $X_{t}, X_{b}$ and $X_{\tau}$ are defined as

$$
X_{t}=A_{t}-\mu \cot \beta, \quad X_{b}=A_{b}-\mu \tan \beta, \quad X_{\tau}=A_{\tau}-\mu \tan \beta,
$$

where $A_{f}(f=t, b, \tau)$ are the trilinear Higgs-sfermion-sfermion couplings and $\mu$ is a MSSM superpotential parameter. For convenience we define in addition the following symbols:

$$
\alpha_{t}=\frac{y_{t}^{2}}{4 \pi}, \quad \alpha_{s}=\frac{g_{3}^{2}}{4 \pi}, \quad \alpha_{\mathrm{em}}=\frac{e^{2}}{4 \pi}, \quad e=\frac{g_{Y} g_{2}}{\sqrt{g_{Y}^{2}+g_{2}^{2}}}, \quad M_{S}^{2}=m_{\tilde{t}_{1}} m_{\tilde{t}_{2}}
$$

where $m_{\tilde{t}_{i}}$ denotes the $i^{\text {th }} \overline{\mathrm{DR}}^{\prime}$ stop mass.

\section{Matching procedure in general}

We begin by recalling a few basic aspects of the effective field theory approach to compute weak-scale observables, such as the pole mass of the Higgs boson, $M_{h}$, in SUSY models in scenarios where the SUSY scale $\Lambda$ is significantly larger than the weak scale. This will help later in characterizing our approach and in comparing it to other approaches.

\footnotetext{
${ }^{2}$ In the calculation of the 2-loop and 3-loop matching corrections presented in the next sections, we neglect $D$-term contributions, as we work in the gauge-less limit. In the calculation of the 1-loop threshold correction $\Delta \lambda^{1 \ell}$, all $D$-term contributions are taken into account.
} 


\subsection{Basics of the effective field theory approach}

In SUSY models with very heavy new particles of mass $\Lambda \gg v$, an observable $O$ can be expanded perturbatively in a three-fold way: in terms of loops (counted by a generic loopcounting parameter $\alpha$ ), large logarithms of the large mass ratio $L \equiv \log (\Lambda / v)$ and a mass suppression factor $v / \Lambda$. For the particular case of a dimensionless observable $O$ which has a tree-level contribution of order $\alpha^{0}$, the leading $n$-loop contribution $(n \geq 0)$ is typically of the form $\alpha^{n} L^{n}$. Subleading/higher-order contributions have more powers of $\alpha$, fewer powers of $L$ and/or additional factors of $v / \Lambda$. Hence, one can write to all orders

$$
O=\sum_{n=0}^{\infty} \sum_{l=0}^{n} \sum_{k=0}^{\infty} c_{n l k} \alpha^{n} L^{l}\left(\frac{v}{\Lambda}\right)^{k},
$$

where the sum of the terms with $n=0$ represent the tree-level contribution $O^{0 \ell}$ and the coefficients $c_{n l k}$ are constants which may contain the parameters of the full model and logarithms of small mass ratios. An effective field theory calculation allows to include all terms at the $m$-th subleading log level,

$$
\alpha^{n} L^{n}, \ldots, \alpha^{n+m} L^{n} \quad \forall n \geq 0 .
$$

Since terms of all loop orders are contained in the sum of these terms, their inclusion is also called "resummation of logarithms". Usually the resummation is achieved by performing the following three steps (see figure 1):

1. Construct a Lagrangian of the effective theory and derive a relation between the running parameters of the full and the effective theory by a matching calculation at the $m$-loop level at the high scale $Q_{\text {match }} \approx \Lambda .^{3}$

2. Use $(m+1)$-loop renormalization group running to evolve the parameters of the EFT from the scale $Q_{\text {match }}$ to the low-energy scale $Q_{\text {low }}$. In this process the large logarithms are resummed to the $\mathrm{N}^{m} \mathrm{LL}$ order.

3. Match the parameters of the EFT at the scale $Q_{\text {low }}$ to observed quantities and compute the observable in question at $m$-loop level.

It is not only crucial to take into account all $m$-loop terms, but it is also important to consistently truncate the perturbation expansion at the $m$-loop order. In particular, it is imperative not to include any spurious $(>m$ )-loop terms enhanced by large logarithms as this would spoil the correct resummation. On the other hand it is allowed to incorporate $m$-loop terms suppressed by powers of $v / \Lambda$ in the $m$-loop matching, i.e. to take into account fixed order terms of the form $\alpha^{m} L^{k} v / \Lambda$. In this way the computation of low-energy observables can be improved by power suppressed terms at fixed loop order. Note, however, that as long as only running of operators of mass dimension $\leq 4$ is used, power-suppressed large logarithms of the form $L^{k} v / \Lambda$ are not resummed [54], i.e. terms of order $\alpha^{n+m} L^{n+(k \leq m)} v / \Lambda$

\footnotetext{
${ }^{3}$ Alternatively one may integrate out the heavy states and derive the Lagrangian of the EFT, from which the relation between the running parameters of the full and the effective theory can be read off.
} 


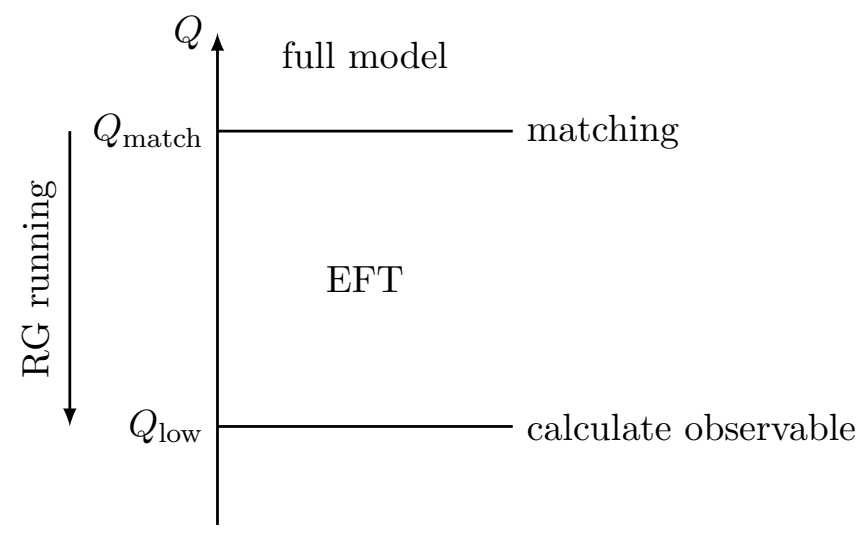

Figure 1. Calculation of an observable in an effective field theory of a full model.

with $n \geq 1$ are not correctly predicted. However, in ref. [44] it was shown that this effect is negligible for the purpose of Higgs pole mass prediction in the relevant parameter space of the MSSM.

\subsection{Parametrization of the matching relations}

In the following we discuss different possibilities to perform the high-scale matching. Specifically, for a matching at some given loop order, one needs to consistently expand either in terms of the running parameter of the fundamental theory $\alpha$ or of the EFT $\hat{\alpha}$. In principle both options are correct and equivalent. However, once perturbation theory is truncated it matters whether truncation is done at the order $(\alpha)^{m}$ or $(\hat{\alpha})^{m}$, because these two kinds of expansions differ by higher-order terms. We give a simple illustration using a 1-loop toy example which is similar to the case of the Higgs pole mass calculation.

We suppose the exact matching condition is given by the equality

$$
\Gamma^{\mathrm{eft}}=\Gamma^{\mathrm{full}},
$$

where $\Gamma$ is some Green function. In the full theory, the 1-loop expression is

$$
\Gamma^{\text {full }}=\alpha+\alpha^{2}\left[\Delta_{\gamma} L+\Delta_{c}\right]
$$

where $\Delta_{\gamma}$ and $\Delta_{c}$ are numerical coefficients. In the EFT, the 1-loop expression reads

$$
\Gamma^{\mathrm{eft}}=\hat{\lambda}+\hat{\alpha}^{2}\left[\Delta_{\gamma} L\right] .
$$

The coefficient $\Delta_{\gamma}$ of the large logarithm $L$ is the same in both cases, because it must cancel in the matching condition. We assume that $\alpha$ and $\hat{\alpha}$ are related at 1-loop level by

$$
\hat{\alpha}=\alpha+\alpha^{2} \Delta_{\alpha}
$$

The matching condition can now be solved perturbatively for $\hat{\lambda}$ in terms of $\alpha$ or $\hat{\alpha}$. At tree-level one obtains

tree-level :

$$
\hat{\lambda}=\alpha=\hat{\alpha}
$$


At the 1-loop level one obtains in terms of $\alpha$ :

$$
\text { full-model parametrization } 1 \ell: \quad \hat{\lambda}=\alpha+\alpha^{2} \Delta_{c}
$$

and in terms of $\hat{\alpha}$ :

$$
\text { EFT parametrization } 1 \ell: \quad \hat{\lambda}=\hat{\alpha}+\hat{\alpha}^{2}\left[\Delta_{c}-\Delta_{\alpha}\right] .
$$

Both expressions (3.8) and (3.9) are valid possibilities for the 1-loop matching relations, but the results for $\hat{\lambda}$ differ by non-log-enhanced 2-loop terms. In fact, this difference could be used as an estimate of the theory uncertainty. For the prediction of the Higgs boson pole mass, the EFT parametrization is used in several calculations such as HSSUSY ${ }^{4}[47,56]$, MhEFT [43] and SusyHD [42], although further parametrizations have been presented in refs. $[23,40]$.

We note that in an algorithmic implementation of the full-model parametrization, the Green function $\Gamma^{\text {full }}$ may be evaluated numerically, while $\Gamma^{\mathrm{eft}}$ needs to be analytically expanded in terms of $\alpha$ and truncated consistently at the 1-loop level. Hence, an analytic manipulation of $\Gamma^{\mathrm{eft}}$ is needed. Conversely, an algorithmic implementation of the EFT parametrization would require an analytic expansion of $\Gamma^{\text {full }}$ in terms of $\hat{\alpha}$ and a consistent truncation of that expansion.

Finally, we note that one might be tempted to plug the respective 1-loop results (3.4)-(3.5) into the matching condition (3.3) to obtain

$$
\hat{\lambda}+\hat{\alpha}^{2}\left[\Delta_{\gamma} L\right]=\alpha+\alpha^{2}\left[\Delta_{\gamma} L+\Delta_{c}\right]
$$

and solve for $\hat{\lambda}$, e.g. numerically. One would then obtain

$$
\text { incorrect: } \quad \hat{\lambda}=\alpha+\alpha^{2} \Delta_{c}+\alpha^{3} 2 \Delta_{\alpha} \Delta_{\gamma} L+\mathcal{O}\left(\alpha^{4}\right) .
$$

Here, a spurious log-enhanced 2-loop term is generated. If such an implementation were used, the resummation of subleading logarithms would be spoiled. A problem of this kind appeared in refs. [54, 55] and a solution was first discussed in ref. [56].

\subsection{Matching of the quartic Higgs coupling}

In the following we will discuss the differences between the two parametrizations in the context of predicting the quartic Higgs coupling $\hat{\lambda}$ from a matching of the Standard Model to the MSSM.

EFT (SM) parametrization. In this parametrization the quartic Higgs coupling $\hat{\lambda}$ is expressed in terms of the $\overline{\mathrm{MS}}$-renormalized SM parameters $\left\{\hat{g}_{1}, \hat{g}_{2}, \hat{g}_{3}, \hat{y}_{t}, \hat{y}_{b}, \hat{y}_{\tau}, \hat{v}\right\}$ at the matching scale $Q_{\text {match }}$. In the scenario with degenerate SUSY mass parameters and $Q_{\text {match }}=M_{S}$, the 1-loop contribution to $\hat{\lambda}$ from stops is given by

$$
\text { EFT parametrization: }\left.\quad \Delta \lambda^{1 \ell}\right|_{\hat{y}_{t}^{4}}=\frac{1}{(4 \pi)^{2}} \hat{y}_{t}^{4} 6\left[x_{t}^{2}-\frac{x_{t}^{4}}{12}\right],
$$

where $x_{t}=X_{t} / M_{S}$ is the dimensionless stop-mixing parameter in the $\overline{\mathrm{DR}}^{\prime}$ scheme.

\footnotetext{
${ }^{4}$ According to ref. [44], the bottom Yukawa coupling inside the 2-loop threshold correction $\Delta \lambda$ are in the full-model parametrization for the reason of correct $\tan \beta$ treatment, as will be discussed in section 5 .
} 
Full-model (MSSM) parametrization. In this parametrization the MSSM parameters are treated as fundamental. At the matching scale the quartic Higgs coupling $\hat{\lambda}$ is then fixed in terms of the MSSM $\overline{\mathrm{DR}}^{\prime}$ parameters. As a result, the 1-loop contribution to $\Delta \lambda^{1 \ell}$ reads at $\mathcal{O}\left(y_{t}^{4}\right)$

$$
\text { full-model parametrization: }\left.\quad \Delta \lambda^{1 \ell}\right|_{y_{t}^{4}}=\frac{1}{(4 \pi)^{2}} y_{t}^{4} s_{\beta}^{4} 6\left[x_{t}^{2}-\frac{x_{t}^{4}}{12}\right],
$$

where $y_{t}$ denotes the MSSM top Yukawa coupling in the $\overline{\mathrm{DR}}^{\prime}$ scheme.

With respect to the top Yukawa and strong gauge coupling, the difference between the EFT and the full-model parametrization (3.12) and (3.13) is of 2-loop order. This can be seen by equivalently reparametrizing eq. (3.13) in terms of the SM $\overline{M S}$ top Yukawa coupling $\hat{y}_{t}$, which leads to

$$
\left.\Delta \lambda^{1 \ell}\right|_{y_{t}^{4}}=\frac{1}{(4 \pi)^{2}} \hat{y}_{t}^{4} 6\left[x_{t}^{2}-\frac{x_{t}^{4}}{12}\right]-\frac{1}{(4 \pi)^{4}} \frac{8}{3} \hat{y}_{t}^{4} \hat{g}_{3}^{2}\left[x_{t}^{5}+\propto x_{t}^{n \leq 4}\right]+\mathcal{O}\left(g_{3}^{4} y_{t}^{4}\right) .
$$

Comparing the two versions of the threshold corrections (3.12) and (3.14) reveals several important points. We note first that by construction the 2-loop term on the r.h.s. of eq. (3.14) does not contain large logarithms, in agreement with the effective field theory paradigm. Clearly, the 2-loop difference between eqs. (3.12) and (3.14) could be used as a measure of the theory uncertainty of the 1-loop prediction of $\hat{\lambda}$ at the matching scale. Finally note that this reparametrization generates a 2-loop $x_{t}^{5}$ term on the r.h.s. of eq. (3.14). This term is correct, i.e. it appears in the explicit 2-loop calculation of ref. [41]. In section 5 we will show that this is not an accident; the full-model parametrization includes important terms correctly, which in the EFT parametrization would require higher-order calculations. It can thus be used to improve the precision of Higgs pole mass prediction in the effective field theory approach.

Automatization of the matching beyond 1-loop level. Besides the higher precision, the full-model parametrization may also be easier to implement in generic spectrum generators that use the FlexibleEFTHiggs approach [54-56]. In this approach the condition

$$
\left(M_{h}^{\mathrm{MSSM}}\right)^{2}=\left(M_{h}^{\mathrm{SM}}\right)^{2}
$$

is numerically solved for $\hat{\lambda}$ at the matching scale. As discussed in ref. [56] and in section 3.2, care has to be taken to avoid the occurrence of spurious large logarithms of higher-order in the matching. A correct application of FlexibleEFTHiggs approach beyond the 1-loop level using the EFT parametrization requires an expansion of the full-model BSM Higgs self-energy $\Sigma_{\phi}^{\mathrm{BSM}}(P)$ in terms of the full-model parameters $P$ and a following expansion of $P$ in terms of the parameters of the EFT (here the SM) $\hat{P}$, including a truncation at some fixed order in $\hat{P}$. This expansion introduces "implicit" terms beyond 1-loop of the form

$$
\text { EFT parametrization: } \quad \Delta \lambda^{2 \ell} \supset-\left(\frac{\partial}{\partial P} \Sigma_{\phi}^{\mathrm{BSM}}\right) \Delta \hat{P}
$$


where $\Delta \hat{P}=P-\hat{P}$ is the threshold correction of SM-like parameters expressed through SM parameters. Thus, the inclusion of derivatives of the BSM Higgs self-energy w.r.t. SM-like parameters becomes mandatory for the cancellation of large logarithms in the matching beyond 1-loop. The calculation of these derivatives requires some extra computational effort, which must be performed for each BSM model. The application of this approach to arbitrary BSM models thus requires some cost.

Within the full-model parametrization, the Higgs self-energy in the EFT, $\Sigma_{h}^{\mathrm{SM}}(\hat{P})$, must be expanded in terms of the parameters of the EFT, $\hat{P}$, which then must be expanded in terms of the parameters of the full model, $P$. As a result, 2-loop structures of the following form are generated

$$
\text { full-model parametrization: } \quad \Delta \lambda^{2 \ell} \supset\left(\frac{\partial}{\partial \hat{P}} \Sigma_{h}^{\mathrm{SM}}\right) \Delta P,
$$

where $\Delta P=\hat{P}-P$ is the threshold correction of SM-like BSM parameters expressed through BSM parameters. Thus, only derivatives of the EFT Higgs self-energy are required. As long as the employed EFT does not change, these derivatives can be computed once and reused in the matching to arbitrary BSM models. With respect to computational effort and model independence, the full-model parametrization is thus advantageous. Due to the reusability of the appearing structures and the improved treatment of $x_{t}($ and $\tan \beta$ ) discussed later, we propose to use the full-model parametrization instead of the EFT parametrization used in HSSUSY, SusyHD and the original FlexibleEFTHiggs implementation [54, 56].

\section{New FlexibleEFTHiggs matching procedure}

In the following we apply the conclusions of the previous section to the matching of the SM to the MSSM and describe a new improved matching procedure of the FlexibleEFTHiggs approach, which also allows to extend the approach beyond the 1-loop level without introducing spurious logarithms of higher order.

\subsection{FlexibleEFTHiggs matching conditions}

The FlexibleEFTHiggs approach is based on the central matching condition

$$
\left(M_{h}^{\mathrm{MSSM}}\right)^{2}=\left(M_{h}^{\mathrm{SM}}\right)^{2}
$$

where $M_{h}^{\text {MSSM }}$ denotes the pole mass of the SM-like Higgs as predicted within the MSSM and $M_{h}^{\mathrm{SM}}$ the Higgs pole mass in the SM as computed in terms of SM parameters. Within the SM, the Higgs pole mass is related to SM tree-level parameters and loop corrections as

$$
\left(M_{h}^{\mathrm{SM}}\right)^{2}=s
$$

where

$$
\begin{aligned}
0 & =s-\hat{m}_{h}^{2}+\operatorname{Re}\left[\Sigma_{h}^{\mathrm{SM}}(s)-\frac{t_{h}^{\mathrm{SM}}}{\hat{v}}\right], \\
\hat{m}_{h}^{2} & =\hat{\lambda} \hat{v}^{2} .
\end{aligned}
$$


Here $\hat{v}$ is the minimum of the loop-corrected SM effective potential and $\Sigma_{h}^{\mathrm{SM}}, t_{h}^{\mathrm{SM}}$ are the $\overline{\mathrm{MS}}$-renormalized self-energy and tadpole, respectively. Within the MSSM, the Higgs pole mass is related to MSSM tree-level parameters and $\overline{\mathrm{DR}}^{\prime}$ renormalized loop corrections as

$$
\left(M_{h}^{\mathrm{MSSM}}\right)^{2}=s, \quad \text { where } \quad 0=\operatorname{det}\left[s \delta_{i j}-\left(m_{\phi}^{2}\right)_{i j}+\operatorname{Re}\left[\Sigma_{\phi, i j}(s)-\frac{t_{\phi, i}}{v_{i}} \delta_{i j}\right]\right] .
$$

Here, the tree-level mass matrix $\left(m_{\phi}^{2}\right)_{i j}$ is parametrized such that the soft-breaking Higgsdoublet mass parameters $m_{H_{u, d}}^{2}$ are eliminated by employing the EWSB equations at the loop level. This elimination introduces the tadpoles $t_{\phi, i}$ on the r.h.s. of eq. (4.5), which are of the same loop order as the momentum-dependent self-energy matrix $\Sigma_{\phi, i j}(s)$ of the BSM model. For later convenience we introduce the abbreviations

$$
\begin{aligned}
\Delta s_{h}^{\mathrm{SM}}\left(p^{2}\right) & =-\operatorname{Re}\left[\Sigma_{h}^{\mathrm{SM}}\left(p^{2}\right)-\frac{t_{h}^{\mathrm{SM}}}{v}\right], \\
\Delta s_{h}^{\mathrm{MSSM}} & =s-m_{h}^{2},
\end{aligned}
$$

where $m_{h}^{2}$ is the SM-like tree-level mass eigenvalue of the matrix $\left(m_{\phi}^{2}\right)_{i j}$. Combining the previous expressions gives rise to the following relation for the SM quartic coupling $\hat{\lambda}$ :

$$
\hat{\lambda}=\frac{1}{\hat{v}^{2}}\left[\left(M_{h}^{\mathrm{MSSM}}\right)^{2}-\Delta s_{h}^{\mathrm{SM}}\left(\left(M_{h}^{\mathrm{MSSM}}\right)^{2}\right)\right] .
$$

This is the master formula for the determination of $\hat{\lambda}$ in the FlexibleEFTHiggs approach; in principle it could be evaluated exactly and at arbitrarily high orders. In particular, it could be evaluated either in the limit $v / M_{S} \rightarrow 0$ or by keeping power-suppressed terms of order $v / M_{S}$. The first option would correspond to the pure EFT approach pursued e.g. in HSSUSY and SusyHD. The second option corresponds to the FlexibleEFTHiggs approach. For an extensive discussion of this method we refer to refs. [54, 56]. As exemplified in appendix A of ref. [54] and in appendix A of the present paper the two options indeed coincide analytically in the limit $M_{S} \rightarrow \infty$.

In the following we evaluate the master formula (4.8) according to the following prescription:

- We use the FlexibleEFTHiggs hybrid method introduced in ref. [54], i.e. we evaluate eq. (4.8) as it stands, including power-suppressed terms of $\mathcal{O}\left(v^{2} / M_{S}^{2}\right)$ arising in the self-energies and tadpoles.

- Eq. (4.8) is evaluated in the full-model (MSSM) parametrization, which is rather easy to generalize to other SUSY models and allows for a resummation of leading $x_{t}$ and $\tan \beta$ contributions in the Yukawa couplings $y_{t}$ and $y_{b}$ as well as in the quartic coupling $\hat{\lambda}$.

- The threshold correction for $\hat{\lambda}$ is calculated at $\mathrm{N}^{3} \mathrm{LO}$ with all 1-loop corrections, 2loop corrections in the gaugeless limit at $\mathcal{O}\left(g_{3}^{2}\left(y_{t}^{4}+y_{b}^{4}\right)+\left(y_{t}^{2}+y_{b}^{2}\right)^{3}+\left(y_{t}^{2}+y_{\tau}^{2}\right)^{3}\right){ }^{5}$

\footnotetext{
${ }^{5}$ Additional 2-loop corrections of $\mathcal{O}\left(v^{2} y_{b}^{4} y_{\tau}^{2}+v^{2} y_{b}^{2} y_{\tau}^{4}\right)$ to the pole mass of the Higgs boson can be found in ref. [19], but we don't include these corrections in the present study.
} 
and 3-loop corrections of $\mathcal{O}\left(g_{3}^{4} y_{t}^{4}\right){ }^{6}$ By including the SM $\beta$-functions up to the 4loop level, this matching allows for a resummation of $\mathrm{N}^{3} \mathrm{LL}$ at the considered order in the couplings. The final Higgs mass prediction will include the complete series of power-suppressed $\left(v^{2} / M_{S}^{2}\right)^{n}$ terms at 1-loop and 2-loop level at the given orders. However, 3-loop suppressed terms are not included in our calculation, because they are neither publicly available in the literature [37] nor implemented in the Himalaya library [33].

In addition to the master formula (4.8), the matching conditions for the other SM parameters $\left\{\hat{g}_{1}, \hat{g}_{2}, \hat{g}_{3}, \hat{y}_{t}, \hat{y}_{b}, \hat{y}_{\tau}, \hat{v}\right\}$ are given by

$$
\begin{aligned}
\left(M_{V}^{\mathrm{MSSM}}\right)^{2} & =\left(M_{V}^{\mathrm{SM}}\right)^{2}, & V=W, Z, \\
M_{f}^{\mathrm{MSSM}} & =M_{f}^{\mathrm{SM}}, & f=t, b, \tau, \\
\Gamma_{\bar{f} f A^{\mu}}^{\mathrm{MSSM}} & =\Gamma_{\bar{f} f A^{\mu},}^{\mathrm{SM}} & \\
\Gamma_{\bar{q} q g_{a}^{\mu}}^{\mathrm{MSSM}} & =\Gamma_{\bar{q} q g_{a}^{\mu},}^{\mathrm{SM}} &
\end{aligned}
$$

where $M$ denotes the pole mass of the corresponding particle and $\Gamma$ is a Green function. The symbols $A^{\mu}$ and $g_{a}^{\mu}$ denote the QED and QCD gauge fields, respectively. Quarks are denoted as $q$ and SM fermions with a non-vanishing electric charge are referred to as $f$.

\subsection{Perturbative expansion of the matching conditions}

In this section we perform the explicit perturbative expansion of the master formula (4.8) and the matching conditions (4.9) in the full-model parametrization. As a result we will obtain all building blocks necessary for the 3-loop Higgs pole mass prediction in the improved FlexibleEFTHiggs approach.

We start by performing the matching at tree-level. At this order one has

$$
\begin{aligned}
& \left(M_{h}^{\mathrm{MSSM}}\right)^{2}=m_{h}^{2}, \quad \Delta s_{h}^{\mathrm{SM}}\left(p^{2}\right)=0, \\
& \left(M_{V}^{\mathrm{MSSM}}\right)^{2}=m_{V}^{2}, \\
& M_{f}^{\mathrm{MSSM}}=m_{f}, \\
& \Gamma_{\bar{f} f A^{\mu}}^{\mathrm{MSSM}}=-e \gamma^{\mu} Q_{f}, \\
& \left(M_{V}^{\mathrm{SM}}\right)^{2}=\hat{m}_{V}^{2}, \\
& V=W, Z \\
& M_{f}^{\mathrm{SM}}=\hat{m}_{f}, \\
& f=t, b, \tau, \\
& \Gamma_{\bar{q} q g_{a}^{\mu}}^{\mathrm{MSSM}}=-g_{3} \gamma^{\mu} T_{a}, \\
& \Gamma_{\bar{f} f A^{\mu}}^{\mathrm{SM}}=-\hat{e} \gamma^{\mu} Q_{f}, \\
& \Gamma_{\bar{q} q g_{a}^{\mu}}^{\mathrm{SM}}=-\hat{g}_{3} \gamma^{\mu} T_{a} .
\end{aligned}
$$

Inserting eqs. (4.10) into the master formula (4.8) and into the matching conditions (4.9) one obtains the $\overline{\mathrm{MS}} \mathrm{SM}$ parameters expressed in terms of $\overline{\mathrm{DR}}^{\prime}$ MSSM parameters at tree level:

$$
\begin{array}{ll}
\hat{\lambda}^{0 \ell}=m_{h}^{2} / v^{2}, & \\
\hat{g}_{i}^{0 \ell}=g_{i}, & i=1,2,3, \\
\hat{y}_{t}^{0 \ell}=y_{t} s_{\beta}, & \\
\hat{y}_{f}^{0 \ell}=y_{f} c_{\beta}, & f=b, \tau, \\
\hat{v}^{0 \ell} & =v .
\end{array}
$$

\footnotetext{
${ }^{6}$ The threshold corrections included in HSSUSY for $\hat{\lambda}$ are of the same order [48], but expressed in the EFT parametrization.
} 
For later convenience we denote the tree-level SM $\overline{\mathrm{MS}}$ parameters on the l.h.s. of eqs. (4.11) as $\hat{P}^{0 \ell}$. At the 1-loop level we obtain accordingly

$$
\begin{aligned}
& \hat{\lambda}^{1 \ell}=\hat{\lambda}^{0 \ell}+\Delta \lambda^{1 \ell}=m_{h}^{2} / v^{2}+\Delta \lambda^{1 \ell}, \\
& \hat{g}_{i}^{1 \ell}=\hat{g}_{i}^{0 \ell}+\Delta g_{i}^{1 \ell}=g_{i}+\Delta g_{i}^{1 \ell}, \quad i=1,2,3, \\
& \hat{y}_{t}^{1 \ell}=\hat{y}_{t}^{0 \ell}+\Delta y_{t}^{1 \ell}=y_{t} s_{\beta}+\Delta y_{t}^{1 \ell}, \\
& \hat{y}_{f}^{1 \ell}=\hat{y}_{f}^{0 \ell}+\Delta y_{f}^{1 \ell}=y_{f} c_{\beta}+\Delta y_{f}^{1 \ell}, \quad f=b, \tau, \\
& \hat{v}^{1 \ell}=\hat{v}^{0 \ell}+\Delta v^{1 \ell}=v+\Delta v^{1 \ell},
\end{aligned}
$$

where the 1-loop threshold corrections on the r.h.s. of eqs. (4.12) are expressed in terms of MSSM $\overline{\mathrm{DR}}^{\prime}$ parameters. In the pure EFT limit $v \rightarrow 0$ the 1-loop threshold corrections can be found for example in refs. [23, 40,41]. The explicit calculation of $\Delta y_{t}^{1 \ell}$ and $\Delta \lambda^{1 \ell}$ beyond the pure EFT limit will be exemplified below. For brevity we denote in the following the 1-loop SM $\overline{\mathrm{MS}}$ parameters on the 1.h.s. of eqs. (4.12) generically as $\hat{P}^{1 \ell}$. Similarly, the $n$-loop SM $\overline{\mathrm{MS}}$ parameters are denoted as $\hat{P}^{n \ell}$. Furthermore we denote the general threshold correction as $\Delta P=\hat{P}-\hat{P}^{0 \ell}$ and specify the notation of a generic $n$-loop threshold correction as

$$
\begin{aligned}
\Delta P^{n \ell} & \equiv \hat{P}^{n \ell}-\hat{P}^{(n-1) \ell}, \\
\Delta P^{\alpha^{n}} & \left.\equiv \Delta P\right|_{\alpha^{n}},
\end{aligned}
$$

which are expressed in terms of MSSM $\overline{\mathrm{DR}}^{\prime}$ parameters, where $\left.\Delta P\right|_{\alpha^{n}}$ denotes all contributions to $\Delta P$ of order $\mathcal{O}\left(\alpha^{n}\right)$.

For the prediction of the SM-like Higgs pole mass in the MSSM with the improved FlexibleEFTHiggs approach up to the order $\mathcal{O}\left(g_{3}^{2}\left(y_{t}^{4}+y_{b}^{4}\right)+\left(y_{t}^{2}+y_{b}^{2}\right)^{3}+\left(y_{t}^{2}+y_{\tau}^{2}\right)^{3}+g_{3}^{4} y_{t}^{4}\right)$, it is sufficient to determine all SM parameters at the 1-loop level, except for $\hat{\lambda}$ and $\hat{y}_{t}$, which must be determined at a higher order. For this reason we describe in the following in more detail the calculation of the threshold corrections $\Delta y_{t}^{n \ell}$ and $\Delta \lambda^{n \ell}$. In order to express these threshold corrections consistently in the full-model parametrization, an extra expansion of the loop corrections in terms of MSSM $\overline{\mathrm{DR}}^{\prime}$ parameters must be performed. We will refer to this procedure as "double loop expansion".

Expansion of the top quark pole mass matching condition. The 2-loop threshold correction for the top Yukawa coupling, $\Delta y_{t}^{2 \ell}$, can be obtained from the top quark pole mass matching condition eq. (4.9b) with

$$
\begin{aligned}
M_{t}^{\mathrm{SM}} & =\hat{m}_{t}+\Delta \hat{m}_{t}^{1 \ell}\left(p=\hat{m}_{t}\right)+\Delta \hat{m}_{t}^{2 \ell}, \\
M_{t}^{\mathrm{MSSM}} & =m_{t}+\Delta m_{t}^{1 \ell}\left(p=m_{t}\right)+\Delta m_{t}^{2 \ell} .
\end{aligned}
$$

The 1-loop corrections on the r.h.s. of eqs. (4.15) are given by

$$
\begin{aligned}
\Delta \hat{m}_{t}^{1 \ell}\left(\hat{m}_{t}\right) & =-\operatorname{Re} \Sigma_{t, S}^{\mathrm{SM}, 1 \ell}-\hat{m}_{t}\left[\operatorname{Re} \Sigma_{t, L}^{\mathrm{SM}, 1 \ell}+\operatorname{Re} \Sigma_{t, R}^{\mathrm{SM}, 1 \ell}+\Delta \hat{m}_{t}^{\mathrm{QCD}, 1 \ell}\right], \\
\Delta m_{t}^{1 \ell}\left(m_{t}\right) & =-\operatorname{Re} \Sigma_{t, S}^{\mathrm{MSSM}, 1 \ell}-m_{t}\left[\operatorname{Re} \Sigma_{t, L}^{\mathrm{MSSM}, 1 \ell}+\operatorname{Re} \Sigma_{t, R}^{\mathrm{MSSM}, 1 \ell}+\Delta m_{t}^{\mathrm{QCD}, 1 \ell}\right], \\
\Delta \hat{m}_{t}^{2 \ell} & =-\hat{m}_{t} \Delta \hat{m}_{t}^{\mathrm{QCD}, 2 \ell} \\
\Delta m_{t}^{2 \ell} & =-m_{t} \Delta m_{t}^{\mathrm{QCD}, 2 \ell}
\end{aligned}
$$


where $\Sigma_{t,\{S, L, R\}}^{\{\mathrm{SM}, \mathrm{MSSM}\}, 1 \ell}$ denote the renormalized scalar, left- and right-handed components of the 1-loop top self-energy evaluated at momentum $p=\hat{m}_{t}$ and $p=m_{t}$ in the SM and MSSM, respectively, without the QCD contributions. The SM self-energies are renormalized in the $\overline{\mathrm{MS}}$ scheme and the MSSM self-energies are renormalized in the $\overline{\mathrm{DR}}^{\prime}$ scheme. In the degenerate SUSY mass limit the 1- and 2-loop QCD contributions are given by [64]

$$
\begin{aligned}
\Delta m_{t}^{\mathrm{QCD}, 1 \ell}= & -\frac{g_{3}^{2}}{(4 \pi)^{2}} \frac{4}{3}\left[5-3 \overline{\log }(t)-x_{t}+\overline{\log }\left(M_{S}^{2}\right)\right] \\
\Delta m_{t}^{\mathrm{QCD}, 2 \ell}= & \frac{g_{3}^{4}}{54(4 \pi)^{4}}\left[1745-640 x_{t}+4 \overline{\log }\left(M_{S}^{2}\right)\left(677-16 x_{t}+93 \overline{\log }\left(M_{S}^{2}\right)\right)\right. \\
& \left.+288 \overline{\log }(t)\left(x_{t}-4 \overline{\log }\left(M_{S}^{2}\right)\right)\right] \\
\Delta \hat{m}_{t}^{\mathrm{QCD}, 1 \ell}= & -\frac{\hat{g}_{3}^{2}}{(4 \pi)^{2}} \frac{4}{3}[4-3 \overline{\log }(\hat{t})] \\
\Delta \hat{m}_{t}^{\mathrm{QCD}, 2 \ell}= & -\frac{\hat{g}_{3}^{4}}{18(4 \pi)^{4}}\left[396 \overline{\log }^{2}(\hat{t})-1452 \overline{\log }(\hat{t})-48 \zeta_{3}+2053+16 \pi^{2}(1+\log 4)\right]
\end{aligned}
$$

where $\hat{t}=\hat{m}_{t}^{2}, t=m_{t}^{2}$ and $\overline{\log }(x) \equiv \log \left(x / Q^{2}\right)$. The 2-loop MSSM QCD contribution $\Delta m_{t}^{\mathrm{QCD}, 2 \ell}$ for non-degenerate SUSY mass parameters can be found in refs. [65-67]. Note that the SM QCD contributions have already been evaluated at $p=M_{t}^{\mathrm{SM}}$ up to order $\mathcal{O}\left(\hat{m}_{t} \hat{g}_{3}^{4}\right)$, while the MSSM QCD contributions have been evaluated at $p=M_{t}^{\text {MSSM }}$ up to order $\mathcal{O}\left(m_{t} g_{3}^{4}\right)$. Thus, the 2-loop contributions $\Delta m_{t}^{2 \ell}$ and $\Delta \hat{m}_{t}^{2 \ell}$ contain terms stemming from momentum iteration out of $\Delta m_{t}^{1 \ell}$ and $\Delta \hat{m}_{t}^{1 \ell}$, respectively.

To obtain the 1- and 2-loop threshold corrections for the top Yukawa coupling in the full-model parametrization, the top quark pole masses (4.15) are inserted into the matching condition $(4.9 \mathrm{~b})$, where both sides must be evaluated at $p=M_{t}^{\mathrm{MSSM}}$. The subsequent expansion of the matching condition in terms of MSSM $\overline{\mathrm{DR}}^{\prime}$ parameters (double loop expansion) is equivalent to taking the expressions in eqs. (4.15) and expanding up to $\mathcal{O}\left(y_{t} g_{3}^{4}\right)$, which yields

$$
\begin{aligned}
& \Delta y_{t}^{1 \ell}=\frac{\sqrt{2}}{v}\left[\Delta m_{t}^{1 \ell}\left(m_{t}\right)-\Delta \hat{m}_{t}^{1 \ell}\left(m_{t}\right)-\frac{m_{t}}{v} \Delta v^{1 \ell}\right], \\
& \Delta y_{t}^{2 \ell}=\frac{\sqrt{2}}{v}\left[\Delta m_{t}^{2 \ell}-\Delta \hat{m}_{t}^{2 \ell}+\sum_{P}\left(\frac{\partial}{\partial P} \hat{m}_{t} \Delta \hat{m}_{t}^{\mathrm{QCD}, 1 \ell}\right) \Delta P\right],
\end{aligned}
$$

with $P \in\left\{\hat{y}_{t}, \hat{g}_{3}\right\}$.

Expansion of the master formula. The $n$-loop threshold correction $\Delta \lambda^{n \ell}$ is obtained from the master formula eq. (4.8). To derive the necessary building blocks to express $\Delta \lambda^{n \ell}$ in the full-model parametrization, the following three expansions must be performed: 
- The prefactor $1 / \hat{v}^{2}$ on the r.h.s. of eq. (4.8) must be expressed in terms of MSSM parameters, which yields ${ }^{7}$

$$
\frac{1}{\hat{v}^{2}}=\frac{1}{v^{2}}\left[1-2 \frac{\Delta v^{1 \ell}}{v}\right]
$$

- The squared Higgs pole mass in the MSSM, $\left(M_{h}^{\mathrm{MSSM}}\right)^{2}$, on the r.h.s. of eq. (4.8) is naturally expanded in terms of MSSM parameters as

$$
\begin{aligned}
\left(M_{h}^{\mathrm{MSSM}}\right)^{2} & =m_{h}^{2}+\Delta s_{h}^{\mathrm{MSSM}, 1 \ell}+\Delta s_{h}^{\mathrm{MSSM}, 2 \ell}+\Delta s_{h}^{\mathrm{MSSM}, 3 \ell}, \\
\Delta s_{h}^{\mathrm{MSSM}, n \ell} & \equiv s^{\mathrm{MSSM}, n \ell}-s^{\operatorname{MSSM},(n-1) \ell},
\end{aligned}
$$

where $s_{h}^{\mathrm{MSSM}, n \ell}$ is obtained from eq. (4.7) with all corrections included up to $n$-loop level. $^{8}$ The 1-loop self-energy and tadpoles can be obtained from SARAH and we expand the self-energy as

$$
\Sigma_{\phi, i j}^{1 \ell}\left(\left(M_{h}^{2}\right)^{\mathrm{MSSM}}\right)=\Sigma_{\phi, i j}^{1 \ell}\left(m_{h}^{2}\right)+\left(\frac{\partial}{\partial p^{2}} \Sigma_{\phi, i j}^{1 \ell}(0)\right) \Delta s_{h}^{\mathrm{MSSM}, 1 \ell} .
$$

Note, that the last term on the r.h.s. of eq. (4.22) contributes pure 2-loop Yukawa terms and thus must be taken into account for a consistent Higgs pole mass prediction at the 2-loop order $\mathcal{O}\left(v^{2}\left(\left(y_{t}^{2}+y_{b}^{2}\right)^{3}+\left(y_{t}^{2}+y_{\tau}^{2}\right)^{3}\right)\right)$, together with the corresponding explicit 2-loop self-energy and tadpole contributions. The explicit 2-loop corrections to $\Delta s_{h}^{\mathrm{MSSM}, 2 \ell}$ read

$$
\Sigma_{\phi, i j}^{2 \ell}(0)-\frac{t_{\phi, i}^{2 \ell}}{v_{i}} \delta_{i j}
$$

which we take in the gaugeless limit at the order $\mathcal{O}\left(v^{2}\left(g_{3}^{2}\left(y_{t}^{4}+y_{b}^{4}\right)+\left(y_{t}^{2}+y_{b}^{2}\right)^{3}+\right.\right.$ $\left.y_{\tau}^{6}\right)$ ) $[10,11,15-17]$. At 3-loop level we include the known MSSM contributions of $\mathcal{O}\left(v^{2} y_{t}^{4} g_{3}^{4}\right)[25,26]$ to the Higgs pole mass,

$$
\Sigma_{\phi, i j}^{3 \ell}(0)-\frac{t_{\phi, i}^{3 \ell}}{v_{i}} \delta_{i j},
$$

which we take from the Himalaya library [33].

- The pure SM contributions $\Delta s_{h}^{\mathrm{SM}}\left(p^{2}\right)$ from eq. (4.6) must also be expressed in terms of MSSM parameters, which we achieve by a double loop expansion:

$$
\Delta s_{h}^{\mathrm{SM}}\left(\left(M_{h}^{\mathrm{MSSM}}\right)^{2}\right)=\Delta s_{h}^{\mathrm{SM}, 1 \ell}\left(m_{h}^{2}\right)+\Delta s_{h}^{\mathrm{SM}, 2 \ell}+\Delta s_{h}^{\mathrm{SM}, 3 \ell},
$$

\footnotetext{
${ }^{7}$ Threshold contributions to $v$ at 2-loop would induce 2-loop contributions in eq. (4.8) beyond the gaugeless limit.

${ }^{8}$ Note that due to the non-linearity of the determinant (4.5), the contribution $\Delta s_{h}^{\mathrm{MSSM}, n \ell}$ contains products of self-energies of lower loop order, which are, however, suppressed by factors of $v^{2} / M_{S}^{2}$ as discussed in refs. [57, 68].
} 
where

$$
\begin{aligned}
\Delta s_{h}^{\mathrm{SM}, 1 \ell}\left(p^{2}\right) & =-\operatorname{Re}\left[\Sigma_{h}^{\mathrm{SM}, 1 \ell}\left(p^{2}\right)-\frac{t_{h}^{\mathrm{SM}, 1 \ell}}{v}\right] \\
\Delta s_{h}^{\mathrm{SM}, 2 \ell} & =-\operatorname{Re}\left[\Sigma_{h}^{\mathrm{SM}, 2 \ell}(0)-\frac{t_{h}^{\mathrm{SM}, 2 \ell}}{v}\right]+\sum_{P}\left(\frac{\partial}{\partial P} \Delta s_{h}^{\mathrm{SM}, 1 \ell}(0)\right) \Delta P^{1 \ell}, \\
\Delta s_{h}^{\mathrm{SM}, 3 \ell} & =-\operatorname{Re}\left[\Sigma_{h}^{\mathrm{SM}, 3 \ell}(0)-\frac{t_{h}^{\mathrm{SM}, 3 \ell}}{v}\right]+\sum_{n \cdot q+m=3}\left(\frac{\partial^{n}}{\partial P^{n}} \Delta s_{h}^{\mathrm{SM}, m \ell}(0)\right)\left(\Delta P^{q \ell}\right)^{n} .
\end{aligned}
$$

The sum on the r.h.s. of eq. (4.26b) runs over $P \in\left\{p^{2}, \hat{y}_{t}, \hat{y}_{b}, \hat{y}_{\tau}, \hat{g}_{3}, \hat{v}\right\}$, where the $\Delta p^{2}$ contribution accounts for the fact that the momentum inserted in the 1-loop SM Higgs mass correction of eq. (4.8) is evaluated at $p^{2}=\left(M_{h}^{\mathrm{MSSM}}\right)^{2}$. Hence the relation

$$
\Delta p^{2}=\Delta s_{h}^{\mathrm{MSSM}, 1 \ell}
$$

includes corrections at 1-loop in the gaugeless limit. The sum on the r.h.s. of eq. (4.26c) runs over $P \in\left\{\hat{y}_{t}, \hat{g}_{3}\right\}$ with $\left(\Delta P^{q \ell}\right)^{n} \in\left\{\Delta y_{t}^{1 \ell}, 1 / 2\left(\Delta y_{t}^{1 \ell}\right)^{2}, \Delta y_{t}^{2 \ell}, \Delta g_{3}^{1 \ell}\right\} .{ }^{9}$ Mixed derivatives and products of threshold corrections do not appear in eq. (4.26c), since they would contribute beyond the considered $\mathcal{O}\left(y_{t}^{4} g_{3}^{4}\right)$ in $\Delta \lambda$. The explicit 2-loop corrections in the effective potential approach at $\mathcal{O}\left(\hat{v}^{2} \hat{y}_{t}^{4} \hat{g}_{3}^{2}\right)$ are taken from refs. [56, 69]. The 2-loop corrections at $\mathcal{O}\left(\hat{v}^{2}\left(\left(\hat{y}_{t}^{2}+\hat{y}_{b}^{2}\right)^{3}+\hat{y}_{\tau}^{6}\right)\right)$ are presented in section 4.3. At 3-loop level, we include contributions of $\mathcal{O}\left(\hat{v}^{2} \hat{y}_{t}^{4} \hat{g}_{3}^{4}\right)$ from ref. [70].

With these ingredients, the expansion of the master formula (4.8) is given by

$$
\begin{aligned}
\hat{\lambda} & =\lambda+\Delta \lambda^{1 \ell}+\Delta \lambda^{2 \ell}+\Delta \lambda^{3 \ell}, \\
\Delta \lambda^{1 \ell} & =\frac{1}{v^{2}}\left[-2 m_{h}^{2} \frac{\Delta v^{1 \ell}}{v}+\Delta s_{h}^{\mathrm{MSSM}, 1 \ell}-\Delta s_{h}^{\mathrm{SM}, 1 \ell}\left(m_{h}^{2}\right)\right], \\
\Delta \lambda^{2 \ell} & =\frac{1}{v^{2}}\left[2 \frac{\Delta v^{1 \ell}}{v}\left(\Delta s_{h}^{\mathrm{SM}, 1 \ell}(0)-\Delta s_{h}^{\mathrm{MSSM}, 1 \ell}\right)+\Delta s_{h}^{\mathrm{MSSM}, 2 \ell}-\Delta s_{h}^{\mathrm{SM}, 2 \ell}\right], \\
\Delta \lambda^{3 \ell} & =\frac{1}{v^{2}}\left[\Delta s_{h}^{\mathrm{MSSM}, 3 \ell}-\Delta s_{h}^{\mathrm{SM}, 3 \ell}\right] .
\end{aligned}
$$

Note that for consistency the parameter shifts $\Delta P$, which contribute to $\Delta \lambda^{2 \ell}$ and $\Delta \lambda^{3 \ell}$, must be evaluated in the gaugeless limit. Note also that the double loop expansion for the 3-loop threshold correction $\Delta \lambda^{3 \ell}$ is relatively simple, because only a small sub-set of implicit corrections contribute at order $\mathcal{O}\left(y_{t}^{4} g_{3}^{4}\right)$.

\subsection{Explicit results and comparison to the literature}

In this subsection we present several explicit results and analytic expressions for the threshold corrections at the 1-loop, 2-loop and 3-loop level. The first purpose is to demonstrate

\footnotetext{
${ }^{9}$ Note that the threshold correction $\left(\Delta P^{q \ell}\right)^{n}$ includes QCD corrections only.
} 
the internal consistency of the new way of setting up the threshold corrections by checking that all explicit large logarithms cancel in eqs. (4.18) and (4.28). A second purpose is to verify the correctness of the results by comparing to results presented in the literature, appropriately reparametrized. Finally, we also present several new analytic results at the 2-loop level.

All results in this subsection are provided in the EFT limit $v^{2} \ll M_{S}^{2}$ and for the degenerate mass case and non-trivial stop mixing.

$\boldsymbol{\Delta} \boldsymbol{y}_{\boldsymbol{t}} \boldsymbol{a t} \mathcal{O}\left(\boldsymbol{y}_{t} \boldsymbol{g}_{3}^{4}\right)$. We start with the derivation of the 1- and 2-loop threshold corrections for the top Yukawa coupling, $\Delta y_{t}^{1 \ell}$ and $\Delta y_{t}^{2 \ell}$, at $\mathcal{O}\left(y_{t} g_{3}^{2 n}\right)$ from eqs. (4.18). The exact 2loop pole mass contribution in the MSSM (self-energy + momentum iteration) is obtained from refs. [66, 67]. The 2-loop pole mass contribution in the SM is taken from ref. [64]. Using the notation as introduced in eq. (4.14) and for the case of degenerate SUSY mass parameters the corrections on the r.h.s. of eqs. (4.18a) and (4.18b) evaluate to

$$
\begin{aligned}
\left.\hat{y}_{t}\right|_{y_{t} \alpha_{s}^{\leq 2}} & =y_{t} s_{\beta}+\Delta y_{t}^{\alpha_{s}}+\Delta y_{t}^{\alpha_{s}^{2}}, \\
\Delta y_{t}^{\alpha_{s}} & =y_{t} s_{\beta} \frac{g_{3}^{2}}{(4 \pi)^{2}} \frac{4}{3}\left[1-x_{t}+\overline{\log }\left(M_{S}^{2}\right)\right], \\
\Delta y_{t}^{\alpha_{s}^{2}} & =y_{t} s_{\beta} \frac{g_{3}^{4}}{(4 \pi)^{4}} \frac{1}{54}\left[2099-832 x_{t}+\left(1748-64 x_{t}\right) \overline{\log }\left(M_{S}^{2}\right)+372 \overline{\log }^{2}\left(M_{S}^{2}\right)\right] .
\end{aligned}
$$

By squaring eq. (4.29a) we obtain perfect analytic agreement with the 2-loop threshold correction presented in ref. [48]. Note that the presented threshold corrections $\Delta y_{t}^{\alpha_{s}}$ and $\Delta y_{t}^{\alpha_{s}^{2}}$ are linear in $x_{t}$, which will be relevant for the determination of $\hat{\lambda}$ in the full-model parametrization below.

For future use we record here the corresponding result in EFT parametrization in an analogous notation as in eq. (4.29):

$$
\begin{aligned}
\left.y_{t}\right|_{\hat{y}_{t} \hat{\alpha}_{s}^{\leq 2}} & =\frac{\hat{y}_{t}}{s_{\beta}}+\Delta \hat{y}_{t}^{\hat{\alpha}_{s}}+\Delta \hat{y}_{t}^{\hat{\alpha}_{s}^{2}}, \\
\Delta \hat{y}_{t}^{\hat{\alpha}_{s}} & =\frac{\hat{y}_{t}}{s_{\beta}} \frac{\hat{g}_{3}^{2}}{(4 \pi)^{2}} \frac{4}{3}\left[-1+x_{t}-\overline{\log }\left(M_{S}^{2}\right)\right], \\
\Delta \hat{y}_{t}^{\hat{\alpha}_{s}^{2}} & =\frac{\hat{y}_{t}}{s_{\beta}} \frac{\hat{g}_{3}^{4}}{(4 \pi)^{4}} \frac{1}{54}\left[-2075+712 x_{t}+96 x_{t}^{2}-\left(1340+416 x_{t}\right) \overline{\log }\left(M_{S}^{2}\right)+12 \overline{\log }^{2}\left(M_{S}^{2}\right)\right] .
\end{aligned}
$$

A noteworthy difference is the term $\propto g_{3}^{4} x_{t}^{2}$, which appears in the EFT parametrized expression in eq. (4.30c), but not in the full-model parametrization in eq. (4.29c). This term originates from an implicit (conversion) correction. Its origin can be traced back to eq. (4.18b), which contains a derivative term of the form

$$
\left(\frac{\mathrm{d}}{\mathrm{d} m_{t}} \Sigma_{t}^{\mathrm{MSSM}, \alpha_{s}}\left(m_{t}, m_{t}\right)\right) \frac{v}{\sqrt{2}} \Delta y_{t}^{\alpha_{s}}
$$

when evaluated in the EFT parametrization. The 1-loop correction $\Delta y_{t}^{\alpha_{s}}$ and the 1-loop MSSM top quark self-energy $\Sigma_{t}^{\mathrm{MSSM}, \alpha_{s}}$ each contain terms $\propto x_{t}$, which results in a contribution $\propto x_{t}^{2}$ in eq. (4.31). As will be discussed below, this 2-loop $x_{t}^{2}$ term is also present 
in the MSSM-parametrized threshold correction, where it is implicitly taken into account by the 1-loop threshold of eq. (4.29b).

$\boldsymbol{\Delta} \boldsymbol{\lambda} \boldsymbol{a t} \mathcal{O}\left(\boldsymbol{g}_{\mathbf{3}}^{\mathbf{2}} \boldsymbol{y}_{\boldsymbol{t}}^{\mathbf{4}}\right)$. We continue and show that eq. (4.28c) in that form leads to the known expression of $\Delta \lambda^{2 \ell}$ at $\mathcal{O}\left(g_{3}^{2} y_{t}^{4}\right)$, presented in ref. [41]. The calculation of this correction from eq. (4.28c) at 2-loop level requires the explicit 2-loop corrections of $\mathcal{O}\left(v^{2} g_{3}^{2} y_{t}^{4}\right)$ for both the SM [69] and MSSM [10] Higgs pole mass. Since the threshold correction to the VEV $\Delta v$ does not contribute at this order, eq. (4.28c) simplifies for $Q=M_{S}$ to

$$
\begin{aligned}
\left.\Delta \lambda^{2 \ell}\right|_{g_{3}^{2} y_{t}^{4}} & =\frac{1}{v^{2}}\left[\Delta s_{h}^{\mathrm{MSSM}, 2 \ell}-\Delta s_{h}^{\mathrm{SM}, 2 \ell}\right] \\
& =\frac{1}{v^{2}}\left[\Delta s_{h}^{\mathrm{MSSM}, y_{t}^{4} g_{3}^{2}}+\operatorname{Re}\left[\Sigma_{h}^{\mathrm{SM}, y_{t}^{4} g_{3}^{2}}(0)-\frac{t_{h}^{\mathrm{SM}, y_{t}^{4} g_{3}^{2}}}{v}\right]-\left(\frac{\partial}{\partial \hat{y}_{t}} \Delta s^{\mathrm{SM}, \hat{y}_{t}^{4}}(0)\right) \Delta y_{t}^{\alpha_{s}}\right] \\
& =\frac{g_{3}^{2} y_{t}^{4} s_{\beta}^{4}}{(4 \pi)^{4}} \frac{4}{3} x_{t}\left[-24+12 x_{t}+4 x_{t}^{2}-x_{t}^{3}\right]
\end{aligned}
$$

where we have used $\Delta y_{t}^{1 \ell}$ from eq. (4.29b). In contrast to the result presented in ref. [41], eq. (4.32c) is expressed in the full-model parametrization. To compare our result with eq. (36) of ref. [41], which is presented in the EFT parametrization, one has to express the SM top Yukawa coupling $\hat{y}_{t}$ in $\Delta \lambda^{1 \ell}$ from eq. (3.12) in terms of the MSSM top Yukawa coupling $y_{t}$. After truncation at the 2-loop order $\mathcal{O}\left(g_{3}^{2} y_{t}^{4}\right)$, the combined expression is identical to eq. (4.32c).

$\boldsymbol{\Delta} \boldsymbol{\lambda}$ at $\mathcal{O}\left(\boldsymbol{y}_{t}^{\mathbf{6}}\right)$. At 2-loop $\mathcal{O}\left(y_{t}^{6}\right)$ we can perform an even stricter consistency test of eq. (4.28c), because the implicit corrections at this order have all non-trivial contributions from eqs. (4.22), (4.26b) and (4.28c). Since the explicit 2-loop contributions in the MSSM [68] and SM [42] are known, the only missing contributions in eq. (4.28c) are the implicit corrections. The 1-loop threshold corrections of $\mathcal{O}\left(\alpha_{t}\right)$ to $P \in\left\{p^{2}, y_{t}, v\right\}$ are obtained from ref. [40]. In appendix A we present all contributions and the explicit derivation in an expansion of $c t_{\beta} \equiv 1 / \tan \beta$ up to the second order for $Q=M_{S}$. Besides this expansion we have also proven the equivalence between our threshold correction and the one presented in ref. [42] for the general $\tan \beta$ dependence. Since the expression from ref. [42] does (by construction) not contain large logarithmic contributions, we have shown that all large logarithmic contributions vanish in eq. (4.28c) at $\mathcal{O}\left(y_{t}^{6}\right)$. Our explicit result for the 2-loop threshold correction in the full-model parametrization reads:

$$
\begin{aligned}
\left.\Delta \lambda^{2 \ell}\right|_{y_{t}^{6}}= & \frac{y_{t}^{6}}{4(4 \pi)^{4}}\left[-4 x_{t}^{6}+53.751 x_{t}^{4}-192.254 x_{t}^{2}-109.503\right. \\
& +c t_{\beta}\left(-0.497 x_{t}^{5}+9.99 x_{t}^{3}-9.015 x_{t}\right) \\
& +c t_{\beta}^{2}\left(11.751 x_{t}^{6}-145.508 x_{t}^{4}+592.02 x_{t}^{2}-317.702\right) \\
& \left.+\mathcal{O}\left(c t_{\beta}^{3}\right)\right]
\end{aligned}
$$

where the analytical result is shown in eq. (A.8). 
$\boldsymbol{\Delta} \boldsymbol{\lambda} \boldsymbol{a t} \mathcal{O}\left(\boldsymbol{y}_{\boldsymbol{t}}^{\mathbf{4}} \boldsymbol{y}_{\boldsymbol{\tau}}^{\mathbf{2}}\right) . \quad$ At $\mathcal{O}\left(y_{t}^{4} y_{\tau}^{2}\right)$ an interesting comparison of threshold contributions in both parametrizations can be made. In a fixed-order calculation there are no 2-loop diagrams which explicitly contribute to the squared Higgs pole mass $M_{h}^{2}$ at $\mathcal{O}\left(v^{2} y_{t}^{4} y_{\tau}^{2}\right)$. However, in a fixed-order calculation contributions of this order are induced by momentum iteration. In an EFT calculation based on EFT parametrization, the contributions of $\mathcal{O}\left(\hat{y}_{t}^{4} \hat{y}_{\tau}^{2}\right)$ to $\Delta \lambda^{2 \ell}$ vanish, see the remarks in section 2 of ref. [44]. However, in our matching procedure, which is based on the full-model parametrization, terms of this order are explicitly generated. This can be seen as follows. Evaluating the implicit correction on the r.h.s. of eq. (4.26b) of $\mathcal{O}\left(y_{t}^{4} y_{\tau}^{2}\right)$ yields

$$
\begin{aligned}
\Delta s_{h}^{\mathrm{SM}, y_{t}^{4} y_{\tau}^{2}} & =\left.\sum_{P}\left(\frac{\partial}{\partial P} \Delta s_{h}^{\mathrm{SM}, 1 \ell}(0)\right) \Delta P^{1 \ell}\right|_{v^{2} y_{t}^{4} y_{\tau}^{2}} \\
& =\left(\frac{\partial}{\partial \hat{y}_{t}} \Delta s_{h}^{\mathrm{SM}, \hat{y}_{t}^{4}}\right) \Delta y_{t}^{\alpha_{\tau}}+\left(\frac{\partial}{\partial \hat{v}} \Delta s_{h}^{\mathrm{SM}, \hat{y}_{t}^{4}}\right) \Delta v^{\alpha_{\tau}}+\left(\frac{\partial}{\partial p^{2}} \Delta s^{\mathrm{SM}, \hat{y}_{\tau}^{4}}\right) \Delta\left(p^{2}\right)^{\alpha_{t}} .
\end{aligned}
$$

Since the tau lepton and stau slepton do not explicitly contribute to the top quark selfenergy at 1-loop level, the threshold corrections $\Delta y_{t}^{\alpha_{\tau}}$ and $\Delta v^{\alpha_{\tau}}$ are related as

$$
\Delta y_{t}^{\alpha_{\tau}}=-\sqrt{2} \frac{m_{t}}{v^{2}} \Delta v^{\alpha_{\tau}}=-y_{t} s_{\beta} \frac{y_{\tau}^{2}}{(4 \pi)^{2}} \frac{c_{\beta}^{2} x_{\tau}^{2}}{12},
$$

where the threshold correction $\Delta v^{\alpha_{\tau}}$ can be obtained from the wave function renormalization of the Higgs, analogously to $\Delta v^{\alpha_{t}}$ out of ref. [40]. Eventually, in the degenerate mass case and $Q=M_{S}$ the threshold correction evaluates to

$$
\begin{aligned}
\left.\Delta \lambda^{2 \ell}\right|_{y_{t}^{4} y_{\tau}^{2}} & =\frac{1}{v^{2}}\left[2 \frac{\Delta v^{\alpha_{\tau}}}{v}\left(\Delta s_{h}^{\mathrm{SM}, y_{t}^{4}}(0)-\Delta s_{h}^{\mathrm{MSSM}, y_{t}^{4}}\right)+\Delta s_{h}^{\mathrm{MSSM}, y_{t}^{4} y_{\tau}^{2}}-\Delta s_{h}^{\mathrm{SM}, y_{t}^{4} y_{\tau}^{2}}\right] \\
& =2 \frac{y_{t}^{4} y_{\tau}^{2}}{(4 \pi)^{4}} s_{\beta}^{4} c_{\beta}^{2} x_{t}^{2} x_{\tau}^{2}\left[-1+\frac{x_{t}^{2}}{12}\right]
\end{aligned}
$$

where $\Delta s^{\mathrm{MSSM}}, y_{t}^{4} y_{\tau}^{2}$ has been computed similarly to eq. (A.2). We note that large logarithmic contributions within the terms of eq. (4.36a) cancel against each other. In order to validate our result from eq. (4.36b), we have computed the analogous expressions in EFT parametrization by performing a reparametrization of the 1-loop correction in eq. (3.12). We find that the EFT-parametrized threshold correction vanishes, which is in line with the remarks in ref. [44]. The discussion of the correction of $\mathcal{O}\left(y_{t}^{2} y_{\tau}^{4}\right)$ is analogous to $\mathcal{O}\left(y_{t}^{4} y_{\tau}^{2}\right)$.

Higgs mass loop corrections at $\mathcal{O}\left(\hat{\boldsymbol{v}}^{2}\left(\left(\hat{\boldsymbol{y}}_{t}^{2}+\hat{\boldsymbol{y}}_{b}^{2}\right)^{3}+\hat{\boldsymbol{y}}_{\tau}^{6}\right)\right)$ in the SM. In the MSSM the 2-loop corrections of $\mathcal{O}\left(v^{2}\left(y_{t}^{2}+y_{b}^{2}\right)^{3}\right)$ to the CP-even Higgs pole mass are known by ref. [17] and are included in several public codes to calculate the CP-even Higgs pole masses in a fixed-order calculation. In the SM, however, the corresponding 2-loop corrections are not available in a simple and explicit form in the literature to our knowledge. ${ }^{10} \mathrm{In}$ order

\footnotetext{
${ }^{10}$ The 2-loop corrections of that order can in principle be calculated from the generic 2-loop effective potential for general renormalizable theories [12].
} 


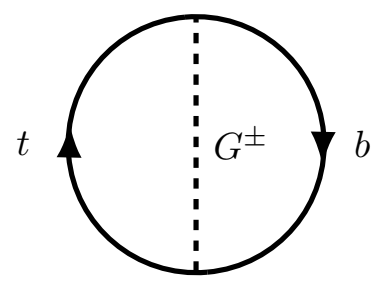

Figure 2. Vacuum bubble diagram in the SM, containing a top quark, a bottom quark and a charged Goldstone boson, which gives rise to 2-loop radiative corrections to the Higgs mass at $\mathcal{O}\left(\hat{v}^{2} \hat{y}_{t}^{4} \hat{y}_{b}^{2} \log ^{2}\left(\hat{v}^{2} / Q^{2}\right)\right)$.

to include the 2-loop threshold corrections of $\mathcal{O}\left(\left(y_{t}^{2}+y_{b}^{2}\right)^{3}\right)$ into the quartic Higgs coupling $\hat{\lambda}$, our approach requires the corresponding fixed-order corrections to be available for both the MSSM and the SM separately. If the contributions in the SM would be omitted, wrong logarithmic enhanced terms of $\mathcal{O}\left(\hat{v}^{2} \hat{y}_{t}^{4} \hat{y}_{b}^{2} \log ^{2}\left(M_{S}^{2} / \hat{m}_{t}^{2}\right)\right)$ would propagate into the expression for the Higgs pole mass. Thus, we calculate here the contributions to the 2-loop effective potential of the SM in the gaugeless limit for a non-vanishing bottom Yukawa coupling. The relevant diagram contributing to the mixed contributions of $\mathcal{O}\left(\hat{v}^{4} \hat{y}_{t}^{4} \hat{y}_{b}^{2}\right)$ is shown in figure 2 .

Following the approach in ref. [15], we compute the 2-loop bubble diagrams $V_{2 \ell}^{\mathrm{SM}}$ and expand the 1-loop effective potential around the $\overline{\mathrm{MS}}$-renormalized masses of the top and bottom quark,

$$
\left.\hat{V}_{2 \ell}^{\mathrm{SM}}\right|_{\left(\hat{y}_{t}^{2}+\hat{y}_{b}^{2}\right)^{3} \phi^{4}}=\left.V_{2 \ell}^{\mathrm{SM}}\right|_{\left(\hat{y}_{t}^{2}+\hat{y}_{b}^{2}\right)^{3} \phi^{4}}+\frac{\partial\left(V_{1 \ell}^{\mathrm{SM}}\right)^{\epsilon}}{\partial \hat{m}_{b}^{2}} \delta \hat{m}_{b}^{2}+\frac{\partial\left(V_{1 \ell}^{\mathrm{SM}}\right)^{\epsilon}}{\partial \hat{m}_{t}^{2}} \delta \hat{m}_{t}^{2}
$$

where $\left(V_{1 \ell}^{\mathrm{SM}}\right)^{\epsilon}$ represents the part of the 1-loop effective potential which is proportional to $(4-D) / 2=\epsilon$ and $\phi$ is a background field. We have checked that $\left.\hat{V}_{2 \ell}^{\mathrm{SM}}\right|_{\left(\hat{y}_{t}^{2}+\hat{y}_{b}^{2}\right)^{3} \phi^{4}}$ is reproduced by using $\left.V_{2 \ell}^{\mathrm{SM}}\right|_{\left(\hat{y}_{t}^{2}+\hat{y}_{b}^{2}\right)^{3} \phi^{4}}$ with the subtracted integrals $\hat{I}$ and $\hat{J}$ instead of $I$ and $J$, which have been introduced in ref. [71]. After expanding the 2-loop integrals around the renormalized Goldstone mass parameter and taking only Yukawa coupling enhanced contributions into account, the finite result expressed in SM $\overline{\mathrm{MS}}$ parameters reads

$$
\begin{aligned}
\left.\hat{V}_{2 \ell}^{\mathrm{SM}}\right|_{\left(\hat{y}_{t}^{2}+\hat{y}_{b}^{2}\right)^{3} \phi^{4}}= & \frac{1}{(4 \pi)^{4}} \frac{3}{2}\left[\phi^{2} \hat{y}_{b}^{4}\left(\hat{I}_{0 T B}+2 \hat{I}_{B B 0}\right)+\phi^{2} \hat{y}_{t}^{4}\left(\hat{I}_{0 T B}+2 \hat{I}_{T T 0}\right)\right. \\
& \left.+2 \hat{y}_{t}^{2}\left(-\phi^{2} \hat{I}_{0 T B} \hat{y}_{b}^{2}+\hat{J}_{T B}+\hat{J}_{T T}\right)+2 \hat{y}_{b}^{2}\left(\hat{J}_{B B}+\hat{J}_{T B}\right)\right] .
\end{aligned}
$$

The squared top and bottom quark mass parameters $T=\hat{y}_{t}^{2} \phi^{2} / 2$ and $B=\hat{y}_{b}^{2} \phi^{2} / 2$ are expressed in terms of the background field $\phi$. In the MSSM, the corresponding SM-like contributions are included in eqs. (3.39) and (3.40) of ref. [13] and we have checked that the effective potential in eq. (4.38) reproduces these results when omitting the corrections from BSM Higgs bosons. The loop corrections to the Higgs pole mass are derived from the 
effective potential by differentiating w.r.t. to the background field $\phi$ as

$$
\begin{aligned}
\Delta m_{h, \mathrm{EP}}^{2, \mathrm{SM}} & =\operatorname{Re}\left[\frac{t_{h}^{\mathrm{SM}, 2 \ell}}{v}-\Sigma_{h}^{\mathrm{SM}, 2 \ell}(0)\right] \\
& =\left.\left(-\frac{1}{\phi} \frac{\partial}{\partial \phi}+\frac{\partial^{2}}{\partial \phi^{2}}\right) \hat{V}_{2 \ell}^{\mathrm{SM}}\right|_{\phi=\hat{v}} .
\end{aligned}
$$

Because the implicit corrections of eq. (4.26b) are analogous to eq. (4.34b), the shift $\Delta s_{h}^{\mathrm{SM}, 2 \ell}$ at $\mathcal{O}\left(\hat{v}^{2}\left(\hat{y}_{b}^{2}+\hat{y}_{t}^{2}\right)^{2}\right)$ is completed by derivatives of eq. (4.38). Neglecting terms of order $\mathcal{O}\left(\hat{y}_{b}^{4} \hat{b}^{2} / \hat{t}\right)$, the loop corrections read

$$
\begin{aligned}
& \left.\Delta m_{h, \mathrm{EP}}^{2, \mathrm{SM}}\right|_{\left(\hat{y}_{t}^{2}+\hat{y}_{b}^{2}\right)^{3} \hat{v}^{2}}=\frac{1}{(4 \pi)^{4}}\left[-2 \hat{y}_{t}^{4} \hat{t}\left(6+\pi^{2}-21 \overline{\log }(\hat{t})+9 \overline{\log }^{2}(\hat{t})\right)\right. \\
& +6 \hat{y}_{t}^{2} \hat{y}_{b}^{2} \hat{t}\left(\pi^{2}+\overline{\log }(\hat{t})+3 \overline{\log }^{2}(\hat{t})\right) \\
& +3 \hat{y}_{b}^{4} \hat{t}\left(-15-2 \pi^{2}+2 \overline{\log }(\hat{t})+6 \overline{\log }^{2}(\hat{t})\right. \\
& \left.+4 \log x_{b t}(2+3 \overline{\log }(\hat{t}))\right) \\
& +\frac{\hat{y}_{b}^{4} \hat{b}}{3}\left(49+6 \pi^{2}+12 \log x_{b t}(5-9 \overline{\log }(\hat{t}))\right. \\
& +18(7-3 \overline{\log }(\hat{t})) \overline{\log }(\hat{t}))]
\end{aligned}
$$

with $\hat{t} \equiv \hat{m}_{t}^{2}, \hat{b} \equiv \hat{m}_{b}^{2}, x_{b t} \equiv \hat{b} / \hat{t}$ and $\overline{\log }(\hat{t}) \equiv \log \left(\hat{t} / Q^{2}\right)$. We note that the first line in eq. (4.40) corresponds to the loop corrections of $\mathcal{O}\left(\hat{y}_{t}^{6} \hat{v}^{2}\right)$, which can be found in ref. [42], and which we reproduce here. In the same way as prescribed in eq. (2.49) of ref. [70], we checked the renormalization scale invariance of the Higgs pole mass at $\mathcal{O}\left(\hat{v}^{2}\left(\hat{y}_{t}^{2}+\hat{y}_{b}^{2}\right)^{3}\right)$ with the contributions of eq. (4.40), which is a non-trivial confirmation of our result.

At $\mathcal{O}\left(\hat{y}_{\tau}^{6} \hat{v}^{2}\right)$ we repeated the calculation in eqs. (4.37) and (4.39b) for the tau and tau neutrino contributions. For massless neutrinos the result is analogous to the first line of eq. (4.40),

$$
\left.\Delta m_{h, \mathrm{EP}}^{2, \mathrm{SM}}\right|_{\hat{y}_{\tau}^{6} \hat{v}^{2}}=-\frac{2 \hat{y}_{\tau}^{4} \hat{\tau}}{(4 \pi)^{4}}\left(2+\frac{\pi^{2}}{3}-7 \overline{\log }(\hat{\tau})+3 \overline{\log }^{2}(\hat{\tau})\right)
$$

with $\hat{\tau} \equiv \hat{m}_{\tau}^{2}$ and $\overline{\log }(\hat{\tau}) \equiv \log \left(\hat{\tau} / Q^{2}\right)$.

$\Delta s_{h}^{\mathrm{SM}, 3 \ell}$ at $\mathcal{O}\left(g_{3}^{4} y_{t}^{4}\right)$. In order to check the consistency of the 3-loop expression of eq. (4.28d), we derive the second term on the r.h.s., $\Delta s_{h}^{\mathrm{SM}, 3 \ell}$, at $\mathcal{O}\left(v^{2} g_{3}^{4} y_{t}^{4}\right)$ in the full-model parametrization and compare it to the result presented in ref. [48]. At 3-loop $\mathcal{O}\left(v^{2} g_{3}^{4} y_{t}^{4}\right)$, the SM Higgs mass correction of eq. (4.26c) receives an explicit self-energy and tadpole contribution, which we take from ref. [70]. We determine the implicit (derivative) contributions of eq. (4.26c) using the 1-loop threshold correction $\Delta g_{3}^{\alpha_{s}}$ from ref. [72] and the 2-loop 
threshold correction $\Delta y_{t}^{\alpha_{s}^{2}}$ form eq. (4.29c). ${ }^{11}$ In the EFT limit and for degenerate SUSY mass parameters our 3-loop Higgs pole mass contribution, expressed in terms of MSSM parameters, reads

$$
\begin{aligned}
\Delta s_{h}^{\mathrm{SM}, y_{t}^{4} g_{3}^{4}}= & \frac{4}{405} \frac{g_{3}^{4} y_{t}^{2}}{(4 \pi)^{4}} t s_{\beta}^{2}\left[-540 \overline{\log }\left(M_{S}^{2}\right)^{2}(86 \overline{\log }(t)+59)\right. \\
& +180 \overline{\log }\left(M_{S}^{2}\right)\left(2 \overline{\log }(t)\left(252 \overline{\log }(t)+88 x_{t}-281\right)+184 x_{t}-557\right) \\
& -32\left(360 \operatorname{Li}_{2}\left(\frac{1}{2}\right)^{2}+120 \pi^{2} \operatorname{Li}_{2}\left(\frac{1}{2}\right)-29 \pi^{4}\right) \\
& -45\left(1536 \operatorname{Li}_{4}\left(\frac{1}{2}\right)-720 \zeta_{3}+3187\right)+59040 x_{t} \\
& -360\left(\overline{\log }(t)\left[4 x_{t}\left(9 x_{t}-22\right)-36 \zeta_{3}+185\right]\right. \\
& \left.\left.+18 \overline{\log }(t)^{2}\left(8 x_{t}-9\right)+207 \overline{\log }(t)^{3}+42 x_{t}^{2}\right)\right]
\end{aligned}
$$

with $t \equiv m_{t}^{2}$ and $\overline{\log }(x) \equiv \log \left(x / Q^{2}\right)$. Our result agrees with eq. (27) of ref. [48]. Furthermore, as a validation of eq. (4.28d), we checked numerically, that inserting eq. (4.42) and the 3-loop MSSM Higgs pole mass contribution from the Himalaya library into eq. (4.28d) reproduces eq. (43) from [48].

\section{Resummation of leading squark mixing contributions}

In the introductory example of section 3.2, differences between the full-model and EFT parametrization were discussed. It was shown that both approaches are equivalent up to higher-order terms, which contribute numerically to the difference of both approaches. From a technical point of view, implementing the full-model parametrized matching is easier to achieve; in this section we present a second, more important argument in favor of this approach: the resummation of higher-order contributions of the full-model squark mixing parameter $x_{f} \equiv X_{f} / M_{S}$ in the context of Higgs mass predictions.

The resummation is analogous to the resummation of large $n$-loop $(\tan \beta)^{n}$-corrections to $m_{b}$ of refs. [73-76], suitably generalized. ${ }^{12}$ We begin here by recalling main features of the $\tan \beta$-resummation in $m_{b}$, rephrase it in the appropriate language and then present the generalization. More details and further generalizations will be presented elsewhere [77].

$\boldsymbol{m}_{\boldsymbol{b}}$-matching and $\tan \boldsymbol{\beta}$-resummation. First we review the resummation of all-order $\tan \beta$-enhanced contributions in the $\overline{\mathrm{DR}}^{\prime}$-renormalized MSSM parameter $m_{b}$. The resummation relies on the following theorem proven in ref. [75]:

\footnotetext{
${ }^{11}$ Note that in this section we ignore contributions of $\mathcal{O}\left(v^{2} / M_{S}^{2}\right)$ for brevity and for cross-checking against expressions from the literature. In our actual implementation of the corrections, presented later, we take all available terms of $\mathcal{O}\left(v^{2} / M_{S}^{2}\right)$ into account.

${ }^{12}$ At this point we want to note that the value of $x_{t}$ is bounded by the necessity of avoiding charge and color breaking minima [41]. For large $m_{\tilde{q}_{3}} \approx m_{\tilde{u}_{3}}$ the absolute value of the dimensionless stop-mixing parameter $\left|x_{t}\right|$ is restricted to be less than 3 , whereas $\tan \beta$ can be as large as 50-60.
} 
There are no contributions to $\Delta m_{b}$ of the order $\mathcal{O}\left(\left(\alpha_{s} \tan \beta \mu / M_{3}\right)^{n}\right)$ for $n \geq 2$.

Here $\Delta m_{b}$ is the loop correction between the $b$-quark pole mass and $\overline{\mathrm{DR}}^{\prime}$ running mass, $M_{b}=m_{b}\left(1+\Delta m_{b}\right)$. The quantity $\Delta m_{b}$ contains a 1-loop term of the order $\alpha_{s} \tan \beta$, terms with lower orders in $\tan \beta$ and terms governed by other couplings, but no higher-loop terms of the orders given in the theorem. The theorem only holds for "unsuppressed" terms, i.e. terms not suppressed by powers of $v / M_{S}$.

The theorem can be equivalently formulated in the language of full-model versus EFT parametrization of the $b$-quark mass matching between the MSSM and the SM. Full-model parametrization means to express the SM $\overline{\mathrm{MS}}$ bottom quark mass $\hat{m}_{b}$ as a perturbative series in the full-model MSSM parameters $m_{b}, \alpha_{s}, \ldots$, and truncating the series at some desired order. The full-model parametrized relation between $\hat{m}_{b}$ and $m_{b}$, truncated at order $\alpha_{s}$ then reads ${ }^{13}$

$$
\left.\hat{m}_{b}\right|_{m_{b} \alpha_{s}^{<1}}=m_{b}\left(1+\Delta m_{b}^{\alpha_{s}}\right)+\cdots
$$

Here the notation of eqs. (4.14) and (4.29a) has been used, and the subscript $m_{b} \alpha_{s}^{\leq 1}$ refers to the full-model parametrization and the chosen truncation order. The dots denote terms irrelevant for the present discussion (containing less powers of $\tan \beta$ and/or power suppressions). In a numerical code, this equation is often numerically solved for $m_{b}$, effectively giving

$$
\left.m_{b}\right|_{m_{b} \alpha_{s}^{\leq 1}}=\frac{\hat{m}_{b}}{1+\Delta m_{b}^{\alpha_{s}}}+\cdots
$$

The point of the theorem is that eq. (5.1) is 1-loop exact with respect to $\left(\alpha_{s} \tan \beta\right)^{n}$-terms and eq. (5.2) correctly takes into account ("resums") all terms of these orders. ${ }^{14}$

On the other hand, in a calculation using the EFT parametrization, $m_{b}$ would be expressed as a perturbative series in terms of $\hat{m}_{b}, \hat{\alpha}_{s}, \ldots,{ }^{15}$ truncated at some desired order. E.g. at order $\hat{\alpha}_{s}$,

$$
\left.m_{b}\right|_{\hat{m}_{b} \hat{\alpha}_{s}^{\leq 1}}=\hat{m}_{b}\left(1-\Delta m_{b}^{\hat{\alpha}_{s}}\right)+\cdots
$$

which contains the correct $\left(\alpha_{s} \tan \beta\right)^{n}$ term only for $n=1$ but misses all higher-order terms. Accordingly, we can evaluate the difference

$$
\left.m_{b}\right|_{m_{b} \alpha_{s}^{\leq 1}}=\left.m_{b}\right|_{\hat{m}_{b} \hat{\alpha}_{s}^{\leq 1}}+\sum_{n \geq 2}\left(-\Delta m_{b}^{\alpha_{s}}\right)^{n}+\cdots
$$

\footnotetext{
${ }^{13}$ As implicitly indicated by ref. [75], a stronger restriction can be formulated which forbids unsuppressed terms of $\mathcal{O}\left(\alpha_{s}^{n} \tan ^{>1} \beta\right)$ in the threshold corrections to the bottom mass matching between the THDM and MSSM.

${ }^{14}$ We note that all codes mentioned in the present paper resum the $m_{b}\left(\alpha_{s} \tan \beta\right)^{n}$ corrections in this way, even if they otherwise do not use full-model parametrization.

${ }^{15}$ For the purpose of the present section the distinction between the SM parameter $\hat{\alpha}_{s}$ and the MSSM parameter $\alpha_{s}$ is not relevant, since the two parameters differ by terms which do not depend on $\tan \beta$ or $x_{f}$.
} 
$\boldsymbol{y}_{\boldsymbol{f}}$-matching and $\boldsymbol{x}_{\boldsymbol{f}}$-resummation. An analogous resummation is possible for the $\alpha_{s} x_{f}$-enhanced contributions to the Yukawa matching for all colored fermions. We note that the factor $\mu \tan \beta$ in the previous theorem arises via $X_{b}=\left(A_{b}-\mu^{*} \tan \beta\right)$. Hence the above theorem generalizes to

There are no unsuppressed contributions to the threshold correction $\Delta y_{f}$ at $\mathcal{O}\left(\alpha_{s}^{n} x_{f}^{>1}\right)$ for $n \geq 1$ in full-model parametrization.

In the following we specialize to the case of the top quark. If we express the SM coupling $\hat{y}_{t}$ as a perturbative series in $y_{t}, \alpha_{s}, \ldots$ and truncate at the order $\alpha_{s}$, we obtain the full-model parametrized expression

$$
\left.\hat{y}_{t}\right|_{y_{t} \alpha_{s}^{\leq 1}}=y_{t} s_{\beta}+\Delta y_{t}^{\alpha_{s}}
$$

The theorem then implies that this relation is 1-loop exact, i.e. there are no unsuppressed higher-order corrections to $\Delta y_{t}^{\alpha_{s}}$ of $\mathcal{O}\left(\alpha_{s}^{n} x_{t}^{>1}\right)$ for $n \geq 2$. In a numerical code such as FlexibleEFTHiggs, eq. (5.5) is solved numerically for $y_{t}$, giving

$$
\left.y_{t} s_{\beta}\right|_{y_{t} \alpha_{s}^{\leq 1}}=\frac{\hat{y}_{t}}{1+\frac{\Delta y_{t}^{\alpha_{s}}}{y_{t} s_{\beta}}},
$$

where the chiral symmetry ensures that for QCD corrections $\Delta y_{t}^{\alpha_{s}} \propto y_{t}$, such that the denominator has the structure $1+\mathcal{O}\left(\alpha_{s} x_{t}\right)+\cdots$. This expression correctly "resums" in particular all terms of the orders $\mathcal{O}\left(\left(\alpha_{s} x_{t}\right)^{n}\right)$. In the EFT parametrization, however, $y_{t}$ is expressed as a perturbative series in $\hat{y}_{t}, \hat{\alpha}_{s}, \ldots$, truncated at some desired order. ${ }^{16}$ The resulting expression is then correct up to that order but misses all higher-order terms of the form $\left(\alpha_{s} x_{t}\right)^{n}$. Similar to eq. (5.4), we can express the difference between the two parametrizations as

$$
\left.y_{t}\right|_{y_{t} \alpha_{s}^{\leq 1}}=\left.y_{t}\right|_{\hat{y}_{t} \hat{\alpha}_{s}^{\leq n}}+\sum_{k>n} a_{k} \hat{y}_{t}\left(\hat{\alpha}_{s} x_{t}\right)^{k}+\cdots
$$

Here the subscript on the r.h.s. denotes the truncation of the EFT parametrization at $n$-loop level, and the dots denote terms irrelevant for the present discussion. The main point is that the difference contains terms of the orders $\left(\alpha_{s} x_{t}\right)^{k}$ with $k>n$, and all these terms are correctly contained in the full-model parametrization but missing in the EFT parametrization, and the coefficients $a_{k}$ can be analytically computed at all orders. As an example of this discussion, we refer to the analysis on the $y_{t}$ matching given in section 4.3: in (4.30c) the explicit 2-loop threshold correction expressed in SM parameters contains the term $\propto \hat{y}_{t}\left(\hat{\alpha}_{s} x_{t}\right)^{2}$. According to eq. (5.7), the MSSM Yukawa coupling in eq. (4.30a) misses terms $\propto \hat{y}_{t}\left(\hat{\alpha}_{s} x_{t}\right)^{\geq 3}$ which, however, are implicitly taken into account in the 1-loop correction of eq. (4.29a).

\footnotetext{
${ }^{16}$ The previous version FlexibleEFTHiggs $1 \ell$ from ref. [56], implemented in FlexibleSUSY 2.0, truncates at 1-loop, while HSSUSY truncates at 2-loop order.
} 
$\hat{\boldsymbol{\lambda}}$-matching and $\boldsymbol{x}_{\boldsymbol{f}}$-resummation. Now we turn to the resummation of $\mathcal{O}\left(g_{3}^{2} x_{t}\right)$ contributions in the matching of $\hat{\lambda}$ in the full-model parametrization. In section 3.3 we already presented an example where the 1-loop matching of $\hat{\lambda}$ and $y_{t}$ in full-model parametrization leads to a correct 2-loop term leading in $x_{t}$. This example illustrates a more general property; however the resummation within $\hat{\lambda}$ is slightly more complicated than the two cases discussed above. We first recall that the threshold correction for $\hat{\lambda}$ in full-model parametrization, truncated at 1-loop order, contains the terms

$$
\Delta \lambda^{1 \ell}=c_{0 t} y_{t}^{4} x_{t}^{4}+c_{01} y_{t}^{2} g_{1}^{2} x_{t}^{2}+c_{02} y_{t}^{2} g_{2}^{2} x_{t}^{2}+\cdots,
$$

where the $c_{0 x}$ are coefficients and the dots denote terms irrelevant for the present discussion. The terms leading in $x_{t}$ are thus of the general form $y_{t}^{2} g_{x}^{2} x_{t}^{m}$ with $g_{x} \in\left\{y_{t}, g_{1}, g_{2}\right\}$ and $m \in\{4,2,2\}$. The resummation of higher-order terms governed by $\left(g_{3}^{2} x_{t}\right)^{n}$ relies on the resummation within the Yukawa coupling discussed before and on the following theorem for the explicit contributions to the threshold corrections:

There are no unsuppressed contributions to $\Delta \lambda$ at $\mathcal{O}\left(y_{t}^{2} g_{x}^{2} g_{3}^{2 n} x_{t}^{>m}\right)$ for $n>0$ in full-model parametrization.

Again the theorem means that the full-model expression (5.8) is 1-loop exact with respect to the leading $\mathcal{O}\left(g_{3}^{2} x_{t}\right)$ terms. The proof of the theorem is analogous to the proof in ref. [75] and relies on the large mass expansion and inspection of individual contributions. For details and generalizations we refer to ref. [77]. If the Yukawa coupling in eq. (5.8) is replaced by $\hat{y}_{t}$ via eq. (5.6), we obtain equations of the form

$$
\left.\Delta \lambda\right|_{y_{t}^{2} g_{x}^{2}}=\hat{c}_{0 x} \hat{y}_{t}^{2} \hat{g}_{x}^{2} x_{t}^{m}+\sum_{n \geq 1} \hat{c}_{n x} \hat{y}_{t}^{2} \hat{g}_{x}^{2} \hat{g}_{3}^{2 n} x_{t}^{m+n}+\cdots,
$$

with appropriately modified coefficients $\hat{c}_{0 x}$ from tree-level matching and higher-order coefficients $\hat{c}_{n x}$. In a numerical code based on the full-model parametrization these higher-order terms are fully taken into account; the coefficients are also fully calculable analytically. All the explicit higher-order terms in eq. (5.9) are correct. In this sense the full-model parametrization resums these terms of $\mathcal{O}\left(\hat{y}_{t}^{2} \hat{g}_{x}^{2} \hat{g}_{3}^{2 n} x_{t}^{m+n}\right)$.

On the other hand, if the $\hat{\lambda}$-matching is done in EFT parametrization, $\Delta \lambda$ is expanded in terms of SM parameters and truncated at some desired order. In that case, leading $\mathcal{O}\left(\hat{g}_{3}^{2} x_{t}\right)$ terms are only taken into account up to that order. The difference between the two versions of the threshold corrections can be written as

$$
\left.\Delta \lambda\right|_{y_{t}^{2} g_{x}^{2}}=\left.\Delta \lambda\right|_{\hat{y}_{t}^{2} \hat{g}_{x}^{2} \hat{g}_{3}^{\leq 2 n}}+\sum_{k>n} \hat{c}_{k x} \hat{y}_{t}^{2} \hat{g}_{x}^{2} \hat{g}_{3}^{2 k} x_{t}^{m+k}+\cdots
$$

All the explicitly given terms $\propto \hat{c}_{k x}$ on the r.h.s. of eq. (5.10) are taken into account correctly in the full-model parametrization but are missing in the EFT parametrization. In other words all these higher-order terms in eq. (5.10) do not arise from explicit multiloop diagrams; instead they only arise via the reparametrization, and the values of the coefficients $\hat{c}_{k x}$ can be deduced from the 1-loop terms leading in $x_{t}$. As an example, we reconsider the concrete calculation in eq. (3.14): the 1-loop matching of $y_{t}$ at $\mathcal{O}\left(g_{3}^{2}\right)$ in 
combination with $\Delta \lambda$ at $\mathcal{O}\left(y_{t}^{4}\right)$ captures the leading $\mathcal{O}\left(\hat{y}_{t}^{4} \hat{g}_{3}^{2} x_{t}^{5}\right)$ contribution of $\Delta \lambda^{2 \ell}$ in EFT parametrization. Likewise, the 3-loop term of $\mathcal{O}\left(\hat{y}_{t}^{4} \hat{g}_{3}^{4} x_{t}^{6}\right)$ is captured, too.

As mentioned before, the theorem from above can be formulated in a more general way, including for example the known structure of $\Delta \lambda^{2 \ell}$ at $\mathcal{O}\left(y_{t}^{6}\right)$ and $\Delta y_{t}^{2 \ell}$ at $\mathcal{O}\left(y_{t}^{3} g_{3}^{2}\right)$, which allows for a resummation of further higher-order corrections [77]. However, we want to emphasize that not all terms with a high power in $x_{t}$ can be resummed. For example, the pure Yukawa $(n+1)$-loop contributions of $\mathcal{O}\left(\hat{y}_{t}^{4+2 n} x_{t}^{4+2 n}\right)$ cannot be captured by the application of neither full-model nor EFT parametrization. The reason is that there exist genuine $(n+1)$-loop diagrams which provide unsuppressed contributions of this order. The numerical impact of missing $\mathcal{O}\left(\hat{y}_{t}^{4+2 n} x_{t}^{4+2 n}\right)$ terms for $n=2$ is discussed in section 7.3 and section 8.3.1.

As an important application and check we consider mixed QCD-electroweak contributions. In codes such as FlexibleEFTHiggs, HSSUSY or SusyHD, the 2-loop and 3-loop Higgs self-energy is only computed in the limit of vanishing electroweak gauge couplings. But the resummation now allows to compute the analytic form of the leading $x_{t}$-terms of the 2 loop and 3-loop mixed QCD-electroweak contributions of the orders $\mathcal{O}\left(\hat{y}_{t}^{2} \hat{g}_{1,2}^{2} \hat{g}_{3}^{2}+\hat{y}_{t}^{2} \hat{g}_{1,2}^{2} \hat{g}_{3}^{4}\right)$. The 1-loop threshold correction $\Delta \lambda^{1 \ell}$ at $\mathcal{O}\left(y_{t}^{2} g_{1,2}^{2}\right)$ originates from $D$-term contributions and involves a maximum $x_{t}$-dependence of order $x_{t}^{2}$. Thus, our prediction for the leading $x_{t}^{3}$ contribution at 2-loop mixed electroweak order is

$$
\begin{aligned}
\left.\Delta \lambda\right|_{\hat{y}_{t}^{2} \hat{g}_{1,2}^{2} \hat{g}_{3}^{2}}= & \frac{\hat{g}_{3}^{2} \hat{y}_{t}^{2}}{(4 \pi)^{4}} \frac{X_{t}^{3}}{M_{3} m_{\tilde{q}_{3}} m_{\tilde{u}_{3}}} \frac{2 c_{2 \beta}}{3} \tilde{F}_{9}\left(\frac{m_{\tilde{q}_{3}}}{M_{3}}, \frac{m_{\tilde{u}_{3}}}{M_{3}}\right) \\
& \times\left[3\left(\tilde{F}_{4}\left(x_{Q U}\right) \hat{g}_{2}^{2}+\tilde{F}_{3}\left(x_{Q U}\right) \hat{g}_{Y}^{2}\right)-c_{2 \beta} \tilde{F}_{5}\left(x_{Q U}\right)\left(\hat{g}_{2}^{2}+\hat{g}_{Y}^{2}\right)\right],
\end{aligned}
$$

with the functions $\tilde{F}_{3,4,5,9}$ defined in ref. [41] with $\tilde{F}_{3,4,5}(1)=\tilde{F}_{9}(1,1)=1$ and $x_{Q U}=$ $m_{\tilde{q}_{3}} / m_{\tilde{u}_{3}}$. Eq. (5.11) is equal to the result obtained in eq. (27) of ref. [49] by an explicit computation of this order in EFT parametrization. The analogous prediction for the leading $x_{t}^{4}$-term of the 3-loop mixed QCD-electroweak contributions is

$$
\begin{aligned}
\left.\Delta \lambda\right|_{\hat{y}_{t}^{2} \hat{g}_{1,2}^{2} \hat{g}_{3}^{4}}= & \frac{\hat{g}_{3}^{4} \hat{y}_{t}^{2}}{(4 \pi)^{6}} \frac{X_{t}^{4}}{M_{3}^{2} m_{\tilde{q}_{3}} m_{\tilde{u}_{3}}} \frac{4 c_{2 \beta}}{9} \tilde{F}_{9}^{2}\left(\frac{m_{\tilde{q}_{3}}}{M_{3}}, \frac{m_{\tilde{u}_{3}}}{M_{3}}\right) \\
& \times\left[3\left(\tilde{F}_{4}\left(x_{Q U}\right) \hat{g}_{2}^{2}+\tilde{F}_{3}\left(x_{Q U}\right) \hat{g}_{Y}^{2}\right)-c_{2 \beta} \tilde{F}_{5}\left(x_{Q U}\right)\left(\hat{g}_{2}^{2}+\hat{g}_{Y}^{2}\right)\right] .
\end{aligned}
$$

All these terms and the corresponding terms of $\geq 4$-loop order are automatically taken into account by the new FlexibleEFTHiggs calculation based on full-model parametrization.

\section{Running and matching procedure at the electroweak scale}

In this section we describe the computations which are performed after the SM parameters are obtained at the high scale by the matching characterized in section 4. As illustrated in figure 1, the subsequent calculation involves two further steps. First the SM parameters are run down to the low-energy electroweak scale by solving the RGEs (subsection 6.1). Second, the low-scale parameters are related to input values of observables, and the final prediction for the Higgs boson mass is computed. The corresponding low-scale matching procedure is described in subsection 6.2. 


\begin{tabular}{|c|c|}
\hline Quantity & Description \\
\hline$M_{t}$ & top quark pole mass \\
\hline$\hat{m}_{b}^{\mathrm{SM}(5)}\left(\hat{m}_{b}^{\mathrm{SM}(5)}\right)$ & $\begin{array}{l}\overline{\mathrm{MS}} \text { bottom quark mass at the scale } Q=\hat{m}_{b} \text { in the SM with } \\
\text { five active quark flavors }\end{array}$ \\
\hline$M_{\tau}$ & $\tau$ lepton pole mass \\
\hline$M_{Z}$ & $Z$ boson pole mass \\
\hline$G_{F}$ & Fermi constant \\
\hline$\hat{\alpha}_{s}^{\mathrm{SM}(5)}\left(M_{Z}\right)$ & $\begin{array}{l}\overline{\mathrm{MS}} \text { strong coupling in the SM with five active quark flavors } \\
\text { at the scale } Q=M_{Z}\end{array}$ \\
\hline$\hat{\alpha}_{\mathrm{em}}^{\mathrm{SM}(5)}\left(M_{Z}\right)$ & $\begin{array}{l}\overline{M S} \text { electromagnetic coupling in the SM with five active quark } \\
\text { flavors at the scale } Q=M_{Z}\end{array}$ \\
\hline
\end{tabular}

Table 1. Low-energy quantities for the determination of SM $\overline{M S}$ parameters.

\subsection{Running to the electroweak scale}

In order to relate the high-scale SM parameters with low-scale SM parameters, the renormalization group equations of the SM are solved numerically. Our new FlexibleEFTHiggs calculation uses the SM $\beta$ functions of refs. [78-85], which include up to 4-loop corrections. The RGEs within the MSSM are not needed for the actual Higgs mass computation. They are only needed if the input scale of MSSM $\overline{\mathrm{DR}}^{\prime}$ parameters does not coincide with the matching scale. In this case our new FlexibleEFTHiggs calculation uses the MSSM 3-loop $\beta$ functions of refs. [86, 87], see also ref. [56].

\subsection{Matching of SM couplings to observables}

In order to express the prediction for the Higgs pole mass in terms of physical quantities, the running SM $\overline{\mathrm{MS}}$ parameters have to be related to observables. There are eight SM $\overline{\mathrm{MS}}$ parameters relevant for the Higgs mass prediction (cf. eq. (2.1)):

$$
\hat{P}=\left\{\hat{g}_{1}, \hat{g}_{2}, \hat{g}_{3}, \hat{y}_{t}, \hat{y}_{b}, \hat{y}_{\tau}, \hat{\lambda}, \hat{v}\right\} .
$$

Among these eight parameters, $\hat{\lambda}$ is fixed by the matching to the MSSM at the SUSY scale, while the other seven parameters are fixed by low-energy observables. Following the approach described in ref. [56], we fix these seven parameters at the scale $Q=M_{Z}$ by relating them to the seven low-energy quantities shown in table 1.

Matching procedure. Before continuing the discussion about the included loop corrections at the electroweak scale, we want to emphasize a qualitative difference of our low-scale matching procedure w.r.t. the procedure described in ref. [56]. In our new FlexibleEFTHiggs matching approach we consider the full-model (MSSM) $\overline{\mathrm{DR}}^{\prime}$ parameters at the SUSY scale to be fundamental. This includes the SUSY parameters and the SM-like full-model parameters $P$ from eq. (2.7). As a consequence, we eventually express all observables, including the low-energy observables from table 1 as well as the predicted Higgs pole mass in terms of the full-model $\overline{\mathrm{DR}}^{\prime}$ parameters. Technically, this is achieved by $(i)$ converting the (fundamental) full-model $\overline{\mathrm{DR}}^{\prime}$ parameters $P\left(M_{S}\right)$ to $\overline{\mathrm{MS}}$ EFT parameters $\hat{P}\left(M_{S}\right)$ 
using the matching conditions (4.8) and (4.9), (ii) renormalization group running of the EFT parameters from the scale $M_{S}$ to the low-energy (electroweak) scale $Q_{\text {low }}$ and (iii) predicting the low-energy quantities $O_{i}^{\text {pred }}$ from table 1 . In the most direct approach the relation between the renormalized SM $\overline{\mathrm{MS}}$ parameters and the observables at the $n$-loop level is constructed as

$$
O_{i}^{\text {pred }}=f_{i}\left(\hat{P}\left(Q_{\text {low }}\right)\right)+\mathcal{O}\left(\hbar^{n+1}\right)
$$

In eq. (6.2) $f_{i}(\hat{P})$ denotes the function that calculates the observable $O_{i}^{\text {pred }}$ as a function of the EFT parameters $\hat{P}$. For a given set of SUSY parameters the SM-like full-model parameters $P$ are adapted such that the predicted observables $O_{i}^{\text {pred }}$ agree with the observed values $O_{i}^{\text {input }}$ up to a sufficiently high precision $\epsilon \ll 1$,

$$
\left|O_{i}^{\text {pred }}-O_{i}^{\text {input }}\right|<\epsilon \quad \forall i .
$$

In contrast, in spectrum generators working in the EFT parametrization (e.g. HSSUSY, SusyHD or MhEFT), the low energy EFT $\overline{\mathrm{MS}}$ parameters $\hat{P}\left(Q_{\text {low }}\right)$ (except for $\hat{\lambda}$ ) are not determined from the full-model parameters, but rather they are directly extracted from the observables as

$$
\hat{P}_{i}\left(Q_{\text {low }}\right)=h_{i}\left(O^{\text {input }}\right)+\mathcal{O}\left(\hbar^{n+1}\right)
$$

with some function $h_{i}$ denoting the calculation. The difference between the EFT parameters $\hat{P}$ from both approaches (6.2) and (6.4) is of higher order. The different higher-order terms depend dominantly on the values of $\hat{P}\left(Q_{\text {low }}\right)$. However, eqs. (6.2) and (6.4) can be modified by higher orders in such a way that the differences expressed in terms of $\hat{P}\left(Q_{\text {low }}\right)$ vanishes, e.g. see the discussion below eq. (6.5). Even if the conditions (6.2) and (6.4) coincide in their inclusion of loop corrections, a remaining difference in the numerical value of $\hat{P}\left(Q_{\text {low }}\right)$ may occur depending on the parametrization of the high-scale matching condition for $\hat{\lambda}$. Though the difference, which originates from reparametrization effects at the high scale, depends only subdominantly on the SUSY parameters, e.g. $\tan \beta .{ }^{17}$

Matching conditions. As described in section 4, we aim for a prediction of the Higgs pole mass at $\mathrm{N}^{3} \mathrm{LO}$ with $\mathrm{N}^{3} \mathrm{LL}$ resummation in QCD. This precision requires to relate the SM $\overline{\mathrm{MS}}$ top quark mass $\hat{m}_{t}$ to the pole mass $M_{t}$ up to $\mathcal{O}\left(\hat{\alpha}_{s}^{3}\right)[88,89]$. We follow the prescription presented in ref. [56] and express $M_{t}$ as

$$
\begin{aligned}
M_{t}= & \hat{m}_{t}-\operatorname{Re} \Sigma_{t, S}^{\mathrm{SM}, 1 \ell}\left(\left(M_{t}^{\mathrm{input}}\right)^{2}, Q\right) \\
& -M_{t}^{\mathrm{input}}\left[\operatorname{Re} \Sigma_{t, L}^{\mathrm{SM}, 1 \ell}\left(\left(M_{t}^{\mathrm{input}}\right)^{2}, Q\right)+\operatorname{Re} \Sigma_{t, R}^{\mathrm{SM}, 1 \ell}\left(\left(M_{t}^{\text {input }}\right)^{2}, Q\right)\right. \\
& \left.+\Delta \hat{m}_{t}^{\mathrm{QCD}, 1 \ell}(Q)+\Delta \hat{m}_{t}^{\mathrm{QCD}, 2 \ell}(Q)+\Delta \hat{m}_{t}^{\mathrm{QCD}, 3 \ell}(Q)+\Delta \hat{m}_{t}^{\mathrm{QCD}, 4 \ell}(Q)\right] .
\end{aligned}
$$

\footnotetext{
${ }^{17}$ Considering the difference of the threshold correction $\Delta \lambda$ between the EFT and the full-model parametrization, the reparametrization effects do explicitly depend on SUSY parameters. Inserted into the $\beta$ functions of the SM, the reparametrization terms receive further loop suppressions. Therefore, the higherorder reparametrization effects which contain MSSM-specific parameters propagate into all EFT parameters at the electroweak scale with additional loop factors.
} 
Note, that we have included the 4-loop QCD contribution from ref. [90] for later use in section 8. In eq. (6.5) $M_{t}^{\text {input }}$ denotes the observed top quark pole mass, $Q$ is the renormalization scale and $\sum_{t,\{L, R, S\}}^{\mathrm{SM}, \ell}\left(p^{2}, Q\right)$ are the left-handed, right-handed and scalar parts of the 1-loop SM top quark self-energy without QCD contributions. The separate SM QCD contributions read [88-90]

$$
\begin{aligned}
\Delta \hat{m}_{t}^{\mathrm{QCD}, 1 \ell}(Q) \approx & \frac{\hat{g}_{3}^{2}}{(4 \pi)^{2}}[4 \overline{\log }(\hat{t})-5.333] \\
\Delta \hat{m}_{t}^{\mathrm{QCD}, 2 \ell}(Q) \approx & \frac{\hat{g}_{3}^{4}}{(4 \pi)^{4}}\left[-6 \overline{\log }^{2}(\hat{t})+38 \overline{\log }(\hat{t})-103.341\right], \\
\Delta \hat{m}_{t}^{\mathrm{QCD}, 3 \ell}(Q) \approx & \frac{\hat{g}_{3}^{6}}{(4 \pi)^{6}}\left[20 \overline{\log }^{3}(\hat{t})-86 \overline{\log }^{2}(\hat{t})+457.747 \overline{\log }(\hat{t})-3458.737\right], \\
\Delta \hat{m}_{t}^{\mathrm{QCD}, 4 \ell}(Q) \approx & \frac{\hat{g}_{3}^{8}}{(4 \pi)^{8}}\left[-85 \overline{\log }^{4}(\hat{t})+323.333 \overline{\log }^{3}(\hat{t})-1832.501 \overline{\log }^{2}(\hat{t})\right. \\
& +45369.45 \overline{\log }(\hat{t})-154481.798],
\end{aligned}
$$

with $\hat{t}=\hat{m}_{t}^{2}$ and $\overline{\log }(x) \equiv \log \left(x / Q^{2}\right)$. As referred to before, the construction of eq. (6.5) contains the observed value of the pole mass $M_{t}^{\text {input }}$, which introduces higher-order terms in eq. (6.2) such that the higher-order difference to the low-scale constraint used in HSSUSY is minimized, see eq. (7) from ref. [56]. The Fermi constant $G_{F}$ is calculated similarly to ref. [72] as

$$
G_{F}=\frac{\pi \hat{\alpha}_{\mathrm{em}}}{\sqrt{2} M_{Z}^{2} \hat{c}^{2} \hat{s}^{2}(1-\Delta \hat{r})},
$$

where $\Delta \hat{r}$ contains only SM contributions, including 2-loop contributions of $\mathcal{O}\left(\hat{\alpha}_{\mathrm{em}} \hat{\alpha}_{s}\right)$ [91]. Because the 2-loop contributions themselves depend on $G_{F}$, an iteration is performed in eq. (6.7). The remaining low-energy quantities from table 1 are calculated at the scale $Q=M_{Z}$ as

$$
\begin{aligned}
\hat{\alpha}_{\mathrm{em}}^{\mathrm{SM}(5)}(Q) & =\frac{\hat{\alpha}_{\mathrm{em}}(Q)}{1+\Delta \hat{\alpha}_{\mathrm{em}}^{1 \ell}(Q)} \\
\hat{\alpha}_{s}^{\mathrm{SM}(5)}(Q) & =\hat{\alpha}_{s}(Q)\left[1-\Delta \hat{\alpha}_{s}^{1 \ell}(Q)-\Delta \hat{\alpha}_{s}^{2 \ell}(Q)-\Delta \hat{\alpha}_{s}^{3 \ell}(Q)\right], \\
M_{\tau} & =\hat{m}_{\tau}(Q)-\operatorname{Re} \Sigma_{\tau}^{1 \ell}\left(\hat{m}_{\tau}, Q\right), \\
M_{Z}^{2} & =\hat{m}_{Z}^{2}(Q)-\operatorname{Re} \Sigma_{Z}^{1 \ell}\left(\hat{m}_{Z}, Q\right) \\
\hat{m}_{b}^{\mathrm{SM}(5)}(Q) & =\hat{m}_{b}(Q)\left[1-\operatorname{Re} \Sigma_{b}^{1 \ell, \text { heavy }}\left(\hat{m}_{b}^{\mathrm{SM}(5)}(Q), Q\right)\right]
\end{aligned}
$$

where the 1-loop correction $\Delta \hat{\alpha}_{\mathrm{em}}^{1 \ell}(Q)$ is parametrized in terms of the 6 flavor $\hat{\alpha}_{\mathrm{em}}(Q)$ as

$$
\Delta \hat{\alpha}_{\mathrm{em}}^{1 \ell}(Q)=-\frac{8}{9 \pi} \hat{\alpha}_{\mathrm{em}}(Q) \log \frac{\hat{m}_{t}(Q)}{M_{Z}} .
$$

The loop corrections of $\Delta \hat{\alpha}_{s}^{n \ell}(Q)$ are defined in eqs. (13)-(15) of ref. [56]. The 1-loop $\overline{\mathrm{MS}}$-renormalized self-energies $\Sigma_{\tau}^{1 \ell}(p, Q)$ and $\Sigma_{Z}^{1 \ell}(p, Q)$ of the $\tau$ lepton and $Z$ boson, respectively, are evaluated at full 1-loop precision. In eq. (6.8e) $\Sigma_{b}^{1 \ell \text {,heavy }}(p, Q)$ denotes the 1-loop 
top quark and electroweak gauge boson contributions to the bottom quark self-energy as described in ref. [62]. The predicted $\hat{m}_{b}^{\mathrm{SM}(5)}(Q)$ is evolved to the scale $Q=\hat{m}_{b}^{\mathrm{SM}(5)}$ to be compared to the input value $\hat{m}_{b}^{\mathrm{SM}(5)}\left(\hat{m}_{b}^{\mathrm{SM}(5)}\right)$.

In principle, one might treat the Higgs mass similar to all other low-scale observables and use it as a constraint in the sense of eq. (6.3) to fix one parameter of the MSSM. However, in our application we choose the Higgs mass at the low scale to be the output of our calculation. Analogous to eq. (1) of ref. [56] we compute the Higgs pole mass in the SM as in eq. (4.3) but instead of using MSSM parameters we express it in terms of SM parameters at $Q=M_{t}^{\text {input }}$. The included corrections are described in section 4 and are of $\mathcal{O}\left(1 \ell+\hat{v}^{2}\left(\hat{g}_{3}^{2}\left(\hat{y}_{t}^{4}+\hat{y}_{b}^{4}\right)+\left(\hat{y}_{t}^{2}+\hat{y}_{b}^{2}\right)^{3}+\left(\hat{y}_{t}^{2}+\hat{y}_{\tau}^{2}\right)^{3}\right)+\hat{v}^{2} \hat{g}_{3}^{4} \hat{y}_{t}^{4}\right)$.

\section{Numerical results}

In this section we present the numerical results for the light CP-even Higgs pole mass calculation in the real MSSM, based on the improved FlexibleEFTHiggs approach. We highlight the numerical impact of the new included threshold corrections and the parametrization scheme and describe in particular the $x_{t}$-resummation.

If not stated otherwise, the dimensionful $\overline{\mathrm{DR}}^{\prime}$-renormalized parameters of the MSSM Lagrangian are set to a common SUSY scale $M_{S}$,

$$
\begin{array}{rlrl}
m_{\tilde{f}_{3}}^{2}\left(M_{S}\right) & =M_{S}^{2}, & & (f=q, u, d, l, e) \\
M_{i}\left(M_{S}\right) & =M_{S}, & & (i=1,2,3) \\
A_{f}\left(M_{S}\right) & =0, & & (f=b, \tau) \\
\mu\left(M_{S}\right) & =M_{S}, & & \\
m_{A}^{2}\left(M_{S}\right) & =\frac{B \mu\left(M_{S}\right)}{\sin \beta\left(M_{S}\right) \cos \beta\left(M_{S}\right)}=M_{S}^{2} .
\end{array}
$$

For scenarios with non-trivial squark mixing we parametrize our results in terms of the dimensionless $\overline{\mathrm{DR}}^{\prime}$ parameter $x_{t} \equiv X_{t} / M_{S}$. In our numerical discussion we choose the input values given in table 2 . Effects from $1^{\text {st }}$ and $2^{\text {nd }}$ generation $(\mathrm{s})$ fermions are omitted in our analysis.

\subsection{Impact of higher orders, the new parametrization and the $x_{t}$-resummation}

We begin with the discussion of the impact of higher-order corrections in the matching and the impact of the new full-model parametrization and the resulting $x_{t}$-resummation. Figure 3 shows the light CP-even Higgs pole mass calculated by different versions of FlexibleEFTHiggs:

- FEFT $1 \ell / 2 \ell / 3 \ell$ : the new FlexibleEFTHiggs hybrid calculation developed in the present paper with full-model (MSSM) parametrization of the matching calculation and $x_{t}$-resummation. The only difference among these calculations stems from the orders taken into account in the calculation of $\hat{\lambda}$, see eq. (4.28). The $1 \ell$ version (blue dotted line) contains all 1 -loop corrections. The $2 \ell$ version (black dashed line) contains in addition the 2-loop contributions of $\mathcal{O}\left(g_{3}^{2}\left(y_{t}^{4}+y_{b}^{4}\right)+\left(y_{t}^{2}+y_{b}^{2}\right)^{3}+\left(y_{t}^{2}+y_{\tau}^{2}\right)^{3}\right)$. The 


\begin{tabular}{|ll|}
\hline Quantity & Value \\
\hline$M_{t}$ & $173.34 \mathrm{GeV}$ \\
$\hat{m}_{b}^{\mathrm{SM}(5)}\left(\hat{m}_{b}^{\mathrm{SM}(5)}\right)$ & $4.18 \mathrm{GeV}$ \\
$M_{\tau}$ & $1.777 \mathrm{GeV}$ \\
$M_{Z}$ & $91.1876 \mathrm{GeV}$ \\
$G_{F}$ & $1.1663787 \cdot 10^{-5} \mathrm{GeV}^{-2}$ \\
$\hat{\alpha}_{s}^{\mathrm{SM}(5)}\left(M_{Z}\right)$ & 0.1184 \\
$\hat{\alpha}_{\mathrm{em}}^{\mathrm{SM}(5)}\left(M_{Z}\right)$ & $1 / 127.944$ \\
\hline
\end{tabular}

Table 2. Low-energy input parameters from refs. [4, 92-95].

$3 \ell$ version (red solid line) contains in addition the 3 -loop contributions of $\mathcal{O}\left(g_{3}^{4} y_{t}^{4}\right)$. All three versions match the top Yukawa coupling at full 1-loop level and 2-loop $\mathcal{O}\left(y_{t} g_{3}^{4}\right)$ at the SUSY scale, whereas the remaining couplings are determined at 1-loop level as described in section 4 . The included corrections to the low-energy input quantities (including the Higgs boson pole mass) are the ones described in section 6 .

- FEFT $1 \ell$ (SM para.): the previous FlexibleEFTHiggs calculation (green dasheddotted line), presented in ref. [56] and included in FlexibleSUSY since version 2.0.0. This calculation is based on the same matching conditions, but employs the EFT (SM) parametrization and includes only 1-loop threshold corrections.

We first discuss the two 1-loop versions (blue dotted and green dashed-dotted lines). They differ essentially by the full-model versus EFT parametrization of the high-scale matching. As discussed in section 5 , in the full-model parametrization certain leading $x_{t}$ terms are correctly taken into account. Eq. (5.9) provides the general form of those terms. To exemplify the correctly included terms, consider the 1-loop threshold correction $\Delta \lambda^{1 \ell}$ in the MSSM parametrization. It contains terms of the order $\mathcal{O}\left(y_{t}^{4} x_{t}^{4}\right)$ and mixed electroweak terms of the form $\mathcal{O}\left(y_{t}^{2} g_{1,2}^{2} x_{t}^{2}\right)$. Upon reparametrization of these contributions in terms of SM parameters, using in particular the 1-loop top Yukawa threshold correction of $\mathcal{O}\left(y_{t} g_{3}^{2} x_{t}\right)$, the terms shown in table 3 are generated. As discussed in section 5 , these terms are not modified by genuine $n$-loop contributions. Hence, already the new FEFT $1 \ell$ calculation correctly takes into account the leading QCD $(n+1)$-loop contributions of the order $x_{t}^{(4+n)}$, and the leading mixed QCD-electroweak $(n+1)$-loop contributions of the order $x_{t}^{(2+n)}$. In contrast, none of the terms in table 3 is correctly taken into account in the previous FEFT $1 \ell$ (SM para.) calculation. As a result of the $x_{t}$-resummation, we see a dramatic shift between the two $1 \ell$ versions in figure 3 .

- Without $x_{t}$-resummation, the FEFT $1 \ell$ (SM para.) result (green dashed-dotted line) and the EFT $3 \ell$ calculation (green solid line) deviate up to $\sim 1.1 \mathrm{GeV}$ for $\left|x_{t}\right|<3$.

- With $x_{t}$-resummation, this deviation between the FEFT $1 \ell$ and FEFT $3 \ell$ calculation decreases to less than $0.3 \mathrm{GeV}$, compare the blue dotted and the red solid lines.

For all values of $x_{t}$ and $M_{S}$, the new FEFT $1 \ell$ calculation is far closer to the FEFT $3 \ell$ calculation; hence the convergence of perturbation theory is significantly improved. 


\begin{tabular}{|ccc|}
\hline loop order & $\Delta \lambda^{\mathrm{QCD}}$ & $\Delta \lambda^{\mathrm{QCD}-\mathrm{EW}}$ \\
\hline $2 \ell$ & $\hat{y}_{t}^{4} \hat{g}_{3}^{2} x_{t}^{5}$ & $\hat{y}_{t}^{2} \hat{g}_{3}^{2} \hat{g}_{1,2}^{2} x_{t}^{3}$ \\
$3 \ell$ & $\hat{y}_{t}^{4} \hat{g}_{3}^{4} x_{t}^{6}$ & $\hat{y}_{t}^{2} \hat{g}_{3}^{4} \hat{g}_{1,2}^{2} x_{t}^{4}$ \\
$4 \ell$ & $\hat{y}_{t}^{4} \hat{g}_{3}^{6} x_{t}^{7}$ & $\hat{y}_{t}^{2} \hat{g}_{3}^{6} \hat{g}_{1,2}^{2} x_{t}^{5}$ \\
$\vdots$ & $\vdots$ & $\vdots$ \\
\hline
\end{tabular}

Table 3. Contributions to $\Delta \lambda^{n \ell}$, which are correctly (implicitly) included by the $x_{t}$-resummation in the new FEFT $1 \ell$ calculation, i.e. terms contained in $\left.\Delta \lambda\right|_{y_{t}^{4}, y_{t}^{2} g_{1,2}^{2}}$. Note, that the FEFT $1 \ell$ calculation is based on the full-model parametrization, but the terms in this table are provided in terms of SM parameters, to compare with other SM-parametrized calculations. Note further, that the terms in this table are already contained in the 1-loop calculation; further, higher-order terms resummed by FEFT $2 \ell / 3 \ell$ are determined by eq. (5.9).

\begin{tabular}{|cccc|}
\hline loop order & $\begin{array}{c}\text { terms contained } \\
\text { in }\left.\Delta \lambda\right|_{y_{t}^{4}}\end{array}$ & $\begin{array}{c}\text { terms contained } \\
\text { in }\left.\Delta \lambda\right|_{\hat{y}_{t}^{4}}\end{array}$ & correct $\Delta \lambda$ \\
\hline $2 \ell$ & $-\frac{1}{2} \kappa^{2} \hat{y}_{t}^{6} x_{t}^{6}$ & 0 & $-\frac{3}{2} \kappa^{2} \hat{y}_{t}^{6} x_{t}^{6}$ \\
$3 \ell$ & $-\frac{9}{16} \kappa^{3} \hat{y}_{t}^{8} x_{t}^{8}$ & 0 & unknown $\cdot \kappa^{3} \hat{y}_{t}^{8} x_{t}^{8}$ \\
$\vdots$ & $\vdots$ & $\vdots$ & $\vdots$ \\
\hline
\end{tabular}

Table 4. Comparison of highest-power $x_{t}$ contributions to $\Delta \lambda$ of $\mathcal{O}\left(\hat{y}_{t}^{2+2 n} x_{t}^{2+2 n}\right)$ at $n$-loop level, implicitly induced by the 1-loop calculations in EFT and full-model parametrization $\left(\kappa=1 /(4 \pi)^{2}\right)$. The second column shows the terms induced in the full-model-parametrized calculation at $\left.\Delta \lambda\right|_{y_{t}^{4}}$. The third column shows the analogous terms in the EFT-parametrized calculation at $\left.\Delta \lambda\right|_{\hat{y}_{t}^{4}}$. The last column represents the correct result in EFT parametrization.

As a side remark we want to stress that in contrast to the correctly included contributions, the $1 \ell$ calculations in both parametrizations fail to capture the pure Yukawa $(n+1)$-loop contributions leading in the stop-mixing parameter of $\mathcal{O}\left(\hat{y}_{t}^{4+2 n} x_{t}^{4+2 n}\right)$. In order to investigate whether those terms counteract the benefits of the resummed QCD-enhanced contributions we show table 4 . The second and third rows contain 2- and 3-loop terms of highest power in $x_{t}$, respectively, which are implicitly contained by the 1-loop calculation in full-model parametrization (second column) and in EFT parametrization (third column). The terms in these columns have been obtained by inverting the 1-loop relation between $y_{t}$ and $\hat{y}_{t}$ in full-model parametrization perturbatively up to the 3-loop level. The terms are compared to the known/unknown correct result in EFT parametrization in the last column. The contributions in the EFT parametrization are vanishing by construction. At 2-loop we see that the implicitly included term from the 1-loop correction in full-model parametrization lies in between the correct result and the analogous one in the EFT parametrization. Without further information from an explicit 3-loop (or higher) calculation there is no indication that the full-model parametrization worsens the convergence of the perturbative expansion with respect to these orders. The 3-loop term is studied numerically 
together with the reparametrization terms from the 2-loop correction $\left.\Delta \lambda^{2 \ell}\right|_{y_{t}^{6}}$ in figure 7 and figure 8.

Second, we discuss the impact of the 2-loop and 3-loop threshold corrections in the new FlexibleEFTHiggs calculation. As can be seen in figure 3, the impact of the higherorder corrections is very small and below $0.3 \mathrm{GeV}$ for all values of $M_{S}$ and $\left|x_{t}\right|<3$ in the shown scenarios. The main reason is again the $x_{t}$-resummation: when going from $1 \ell$ to $2 \ell$, the $1 \ell$ calculation already contains the leading 2 -loop QCD $x_{t}^{5}$ term of table 3 , and the actual $2 \ell$ calculation only adds subleading $x_{t}^{\leq 4}$ terms. Similarly, one can show that the $2 \ell$ calculation already correctly contains the leading 3-loop QCD $x_{t}^{5}$ and $x_{t}^{6}$ terms, and the actual $3 \ell$ calculation only adds subleading $x_{t}^{\leq 4}$ terms. ${ }^{18}$

\subsection{Comparison to state-of-the-art calculations}

In this subsection we compare our new improved FlexibleEFTHiggs calculation with the two state-of-the-art 3-loop fixed-order and EFT calculations from refs. [33, 48]. Both of these calculations are also based on the FlexibleSUSY framework [56, 62], which facilitates the comparison. In detail, these calculations are:

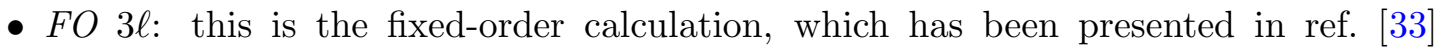
(dashed-double-dotted magenta line in figure 4). It includes loop corrections to the Higgs pole mass in the full-model (MSSM) parametrization at full 1-loop level and 2- and 3-loop corrections in the gaugeless limit at $\mathcal{O}\left(v^{2}\left(g_{3}^{2}\left(y_{t}^{4}+y_{b}^{4}\right)+\left(y_{t}^{2}+y_{b}^{2}\right)^{3}+\right.\right.$ $\left.\left.\left(y_{t}^{2}+y_{\tau}^{2}\right)^{3}\right)\right)$ and $\mathcal{O}\left(v^{2}\left(g_{3}^{4} y_{t}^{4}\right)\right)$, respectively.

- EFT 3 $\ell$ : this calculation is the pure EFT calculation from ref. [48], where a matching at the SUSY scale is performed in the EFT (SM) parametrization (dashed-dotted green line in figure 4). The threshold correction $\Delta \lambda$ includes the known 1-loop contributions from ref. [41], 2-loop contributions at $\mathcal{O}\left(\hat{g}_{3}^{2}\left(\hat{y}_{t}^{4}+\hat{y}_{b}^{4}\right)+\left(\hat{y}_{t}^{2}+\hat{y}_{b}^{2}+\hat{y}_{\tau}^{2}\right)^{3}\right)$ from ref. [44] and 3-loop contributions at $\mathcal{O}\left(\hat{g}_{3}^{4} \hat{y}_{t}^{4}\right)$ from ref. [48], all expressed in terms SM parameters. Note, that this calculation neglects all suppressed $v^{2} / M_{S}^{2}$ terms.

Note, that these two 3-loop calculations take into account loop corrections at the same orders as the presented new FEFT $3 \ell$ calculation, except for 2-loop terms suppressed by powers of $y_{b}^{n} y_{\tau}^{m}$, which are only included in the pure EFT calculation.

The left column of figure 4 provides a first overview of the behavior of the three calculations, for large and small $M_{S}$ and large and small $x_{t}$. The figure confirms the expected behavior: for large $M_{S}$, the pure EFT $3 \ell$ calculation and the hybrid FEFT $3 \ell$ calculation agree well, while the FO $3 \ell$ calculation deviates by several GeV. For small $M_{S}$, the hybrid FEFT $3 \ell$ calculation agrees well with the FO $3 \ell$ calculation, while the pure EFT $3 \ell$ calculation deviates by several GeV. The kinks in the 3-loop lines in the middle left plot around $M_{S} \approx 750 \mathrm{GeV}$ are due to a switch of the mass hierarchy in the 3-loop calculation of Himalaya. The kink in the 3-loop FO prediction of the bottom right plot at

\footnotetext{
${ }^{18}$ The kink in the 3-loop line of the lower left plot around $M_{S} \approx 750 \mathrm{GeV}$ is due to a hierarchy switch in the 3-loop calculation of Himalaya.
} 

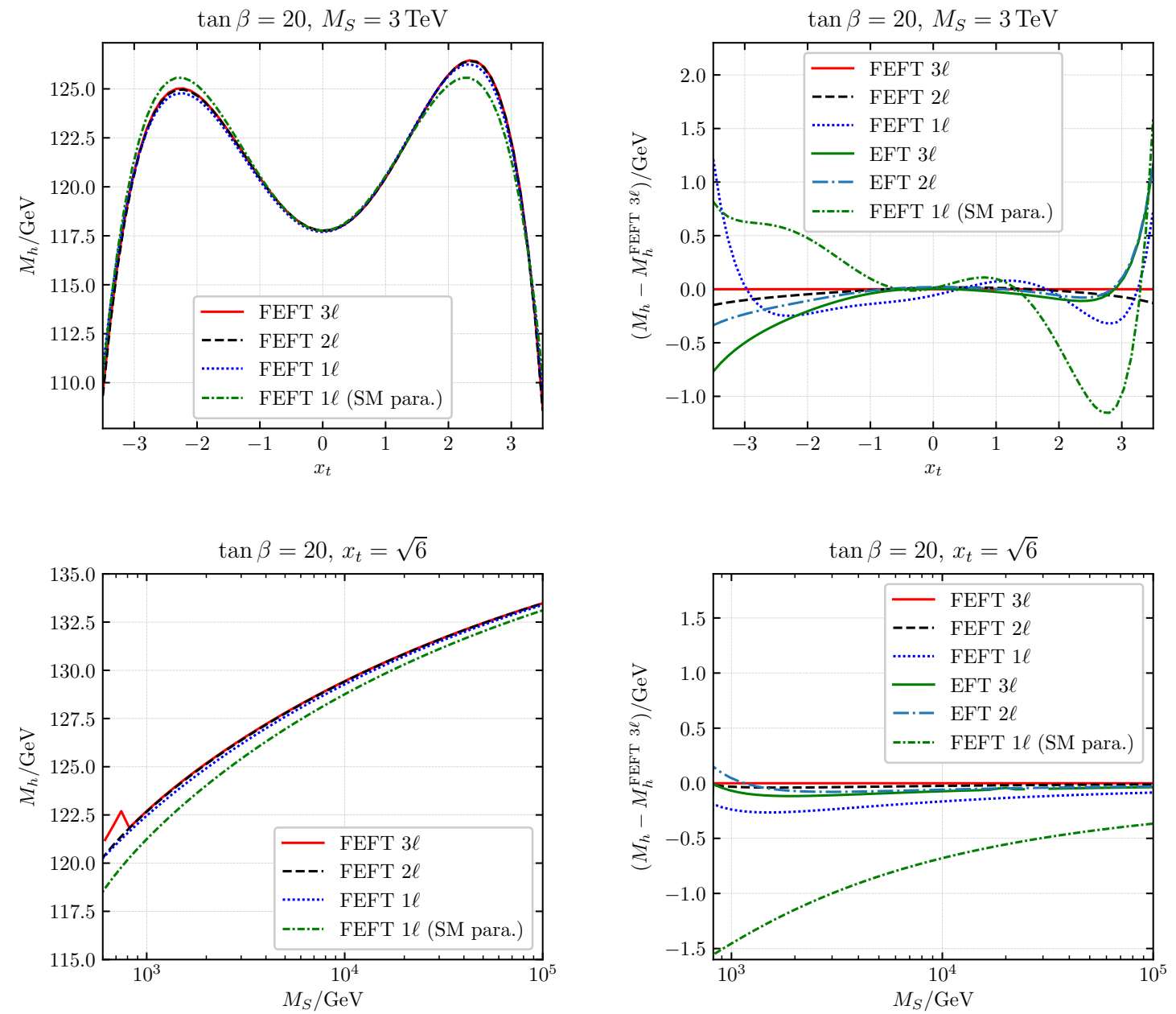

Figure 3. Prediction of the light CP-even Higgs pole mass in the MSSM as computed by the FEFT $1 \ell$ (SM para.) calculation as implemented in FlexibleSUSY 2.4 (green dashed-dotted line) and by the new FEFT calculations at 1-, 2- and 3-loop precision in the $\hat{\lambda}$ matching for $\tan \beta=20$. In the left panels we show the absolute value of $M_{h}$ and in the right panels we show the difference w.r.t. the FEFT $3 \ell$ prediction. On the right panels we show additional lines [48], EFT $2 \ell$ (green solid) and EFT $3 \ell$ (turquoise), which represent the EFT calculation in EFT parametrization at 2-loop and 3-loop respectively (see section 7.2 for the characterization of EFT $3 \ell$ ).

small $\left|x_{t}\right|$ originates from the occurrence of tachyonic running $\overline{\mathrm{DR}}^{\prime}$ Higgs boson masses at the electroweak scale, see the discussion in ref. [60].

The right column of figure 4 shows the differences between the calculations in more detail. In the following we discuss these differences. We first focus on the differences between the new FlexibleEFTHiggs and the pure EFT calculation at SUSY scales above a few TeV. For such values of $M_{S}$, the power-suppressed $v^{2} / M_{S}^{2}$-terms included in the FEFT hybrid calculation are numerically insignificant. Further investigations revealed that the numerical impact of the mixed $\mathcal{O}\left(y_{t}^{4} y_{\tau}^{2}\right)$ threshold corrections included in FEFT are of the order $\Delta M_{h} \approx 10 \mathrm{MeV}$ for $x_{t}=-\sqrt{6}, \tan \beta=20$ and $M_{S}=2 \mathrm{TeV}$ and the additional 

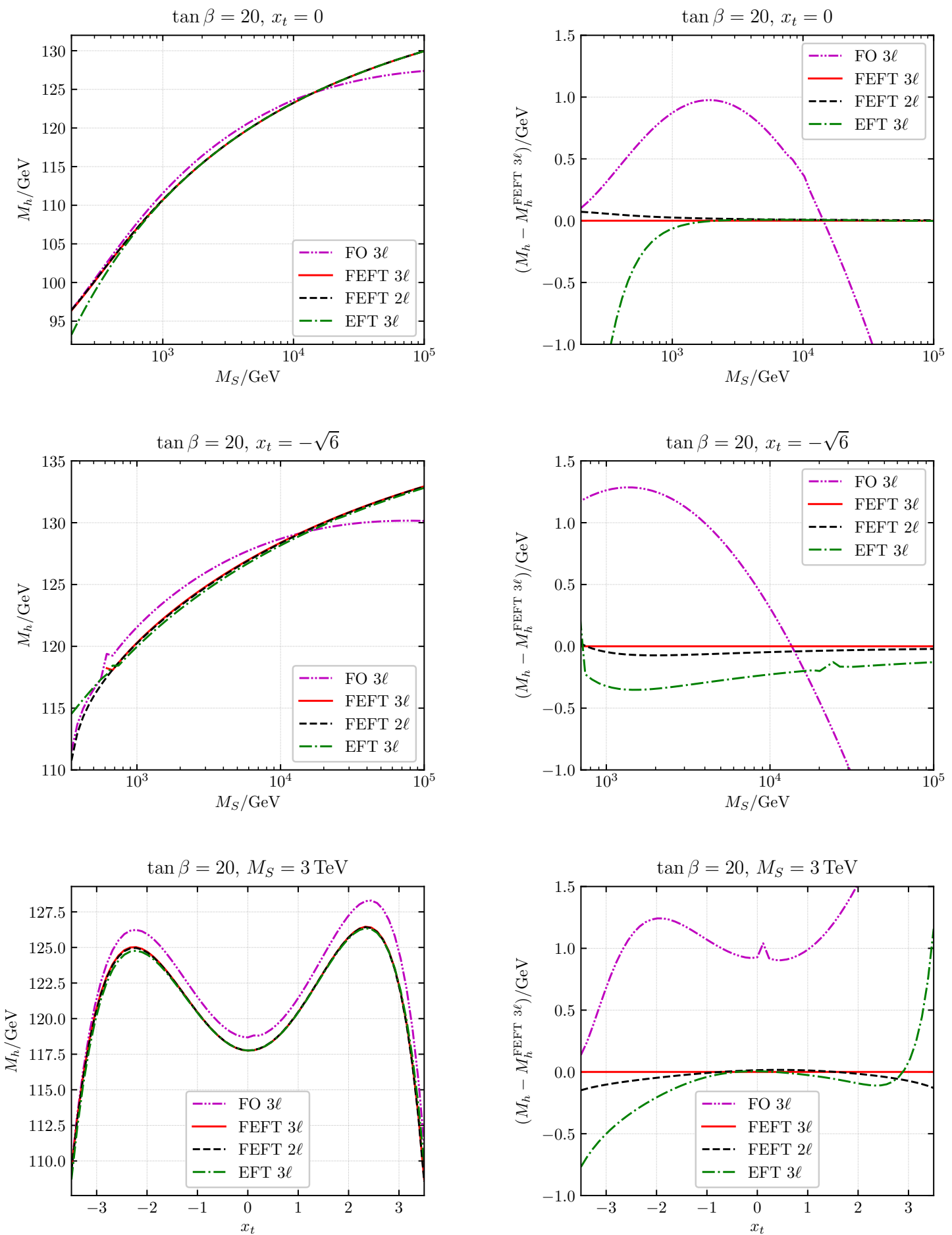

Figure 4. Prediction of the lightest CP-even Higgs pole mass in the MSSM as a function of $x_{t}$ and $M_{S}$ for $\tan \beta=20$. In the left panels we show the absolute value of $M_{h}$ and in the right panels the difference to the FEFT $3 \ell$ prediction. 
corrections in the EFT calculation $\mathcal{O}\left(\hat{y}_{b}^{4} \hat{y}_{\tau}^{2}+\hat{y}_{b}^{2} \hat{y}_{\tau}^{4}\right)$ have an impact of $\Delta M_{h} \approx 10^{-3} \mathrm{MeV}$ and are thus negligible. The essential difference between the FEFT $3 \ell$ calculation (red line) and EFT $3 \ell$ (green dashed-dotted line) is the different parametrization of the matching in terms of either MSSM or SM parameters, and the resulting leading higher-order $x_{t}$ terms included in FEFT $3 \ell$. The precise origin of this difference in the threshold correction $\Delta \lambda$ can be inferred from table 3 . The FEFT $3 \ell$ calculation correctly includes all terms of the table, while the EFT $3 \ell$ calculation only includes the terms of the left column at most up to the 3-loop level, but neither includes the 4-loop term nor any term of the right column. As will be shown in the following subsection, the numerically dominant effect comes from the mixed QCD-EW 2-loop terms of the form $\left(\lambda^{\text {FEFT } 3 \ell}-\lambda^{\text {EFT } 3 \ell}\right) \supset \hat{y}_{t}^{4} \hat{g}_{1,2}^{2} \hat{g}_{3}^{2} x_{t}^{3}$. As shown in the middle row of figure 4 , the numerical difference originating mainly from these terms remains around $200 \mathrm{MeV}$ for $M_{S}=100 \mathrm{TeV}$ (and $x_{t}=-\sqrt{6}$ ). ${ }^{19}$ On the other hand, the lowest row of figure 4 shows that for fixed $M_{S}$ the numerical difference is below $200 \mathrm{MeV}$ for $\left|x_{t}\right| \leq 2$ and $M_{S}=3 \mathrm{TeV}$, but for larger $\left|x_{t}\right|$ the difference rises strongly.

Secondly, we focus on the comparison between the fixed-order and the FlexibleEFTHiggs calculations for SUSY scales below around $1 \mathrm{TeV}$, where both calculations should be valid. By construction, both calculations include the same Higgs pole mass contributions of the orders $\mathcal{O}\left(1 \ell+v^{2}\left(g_{3}^{2}\left(y_{t}^{4}+y_{b}^{4}\right)+\left(y_{t}^{2}+y_{b}^{2}\right)^{3}+\left(y_{t}^{2}+y_{\tau}^{2}\right)^{3}\right)+v^{2}\left(g_{3}^{4} y_{t}^{4}\right)\right)$, including terms suppressed by $v^{2} / M_{S}^{2}$. However, they differ at other orders. Numerically, the difference is below $0.5 \mathrm{GeV}$ for small $x_{t}$ and $M_{S} \lesssim 500 \mathrm{GeV}$ (see top row of figure 4 ), but the difference reaches around $1 \mathrm{GeV}$ for large $\left|x_{t}\right|$ and small $M_{S}$ (see middle row of figure 4 ). The origins of these differences are the following:

- Parametrization: in contrast to our hybrid approach, the determination of the $\overline{\mathrm{DR}}^{\prime}$ MSSM top quark mass $m_{t}$ in the fixed-order calculation consists of the following expanded version of the exact relation

$$
m_{t}=M_{t}\left(1+\Delta m_{t}^{1 \ell}+\Delta m_{t}^{2 \ell}\right),
$$

where $\Delta m_{t}^{1 \ell, 2 \ell}$ represent the 1- and 2-loop corrections to the $\overline{\mathrm{DR}}^{\prime}$ top quark mass as described in refs. $[47,56]$. Analogously to section 5, eq. (7.2) does not represent an all order resummation of terms in the top mass parameter of $m_{t} \supset \hat{m}_{t} \times\left(\hat{g}_{3}^{2} x_{t}\right)^{n}$. Consequently, eq. (7.2) does not lead to an all order resummation of terms in the Higgs pole mass of the form

$$
\left(M_{h}^{\mathrm{FEFT}} 3 \ell\right)^{2}-\left(M_{h}^{\mathrm{FO}} 3 \ell\right)^{2} \supset \hat{m}_{t}^{2}\left(\hat{y}_{t}^{2} x_{t}^{4}+\hat{g}_{1,2}^{2} x_{t}^{2}\right)\left(\hat{g}_{3}^{2} x_{t}\right)^{n}
$$

for $n>2$. Besides these non-resummed terms, our new FlexibleEFTHiggs hybrid calculation includes further incomplete higher-order contributions with high powers in $x_{t}$, which will be discussed in section 8 .

- Momentum iteration: the double loop expansion in our Higgs pole-mass matching condition (4.1) made it necessary to strictly truncate the momentum iteration in order

\footnotetext{
${ }^{19}$ Since the couplings $\hat{y}_{t}$ and $\hat{g}_{3}$ are asymptotically free, the difference between the calculations does not approach a constant but shrinks slowly for higher $M_{S}$.
} 
to avoid incomplete contributions, which could potentially spoil the resummation of the large logarithms. The FO $3 \ell$ calculation, however, does partially include higherorder effects by numerically solving eq. (4.5) for $M_{h}^{2}$. This includes non-logarithmic contributions, for example from the 2-loop electroweak sector and 3-loop top-Yukawa enhanced contributions of the form

$$
\left(M_{h}^{\mathrm{FO} 3 \ell}\right)^{2}-\left(M_{h}^{2, \mathrm{FEFT} 3 \ell}\right)^{2} \supset m_{t}^{2}\left(y_{t}^{2} g_{1,2}^{2} x_{t}^{4}+y_{t}^{4} g_{3}^{2} x_{t}^{6}+y_{t}^{6} x_{t}^{8}\right) .
$$

- log-resummation: for low SUSY scales, the smallness of $\log \left(M_{S} / m_{t}\right)$ leads to a suppression of the resummed tower of large logarithms. However, additional factors of $x_{t}$ might counteract this effect, which potentially increase the relevance of the resummed logarithms, which are correctly included in the EFT-based approaches, such as

$$
\left(M_{h}^{\mathrm{FEFT}} 3 \ell\right)^{2}-\left(M_{h}^{\mathrm{FO}} 3 \ell\right)^{2} \supset \hat{m}_{t}^{2}\left(\hat{y}_{t}^{2} \hat{g}_{1,2}^{2} x_{t}^{2}+\hat{y}_{t}^{4} \hat{g}_{3}^{2} x_{t}^{5}+\hat{y}_{t}^{6} x_{t}^{6}\right) \log \frac{M_{S}}{\hat{m}_{t}} .
$$

\subsection{Further details on the comparison of hybrid and pure EFT calculations}

In the lower-right panel of figure 4 one can see a deviation between the hybrid FEFT $3 \ell$ calculation and EFT $3 \ell$ for large $\left|x_{t}\right|$. In the following we elaborate on the large- $x_{t}$ behavior in more detail.

For the discussion it is sufficient to consider the 2-loop calculations. Figure 5 shows the Higgs pole mass of different 2-loop calculations w.r.t. the FEFT $2 \ell$ calculation (red solid line). The black dashed line corresponds to the same 2-loop calculation, where 2-loop threshold corrections proportional to powers of $y_{b}$ and/or $y_{\tau}$ have been omitted. One finds that the difference between these lines is smaller than $50 \mathrm{MeV}$ for the shown parameter scenario. The blue dotted line represents a modified calculation of the black dashed line, where the 2-loop threshold correction to $\hat{\lambda}$ has been replaced by the analytic 2-loop expressions from eqs. (4.32c) and (4.33) of the order $\mathcal{O}\left(g_{3}^{2} y_{t}^{4}+y_{t}^{6}\right)$, where terms of $\mathcal{O}\left(v^{2} / M_{S}^{2}\right)$ have been neglected. Thus, the difference between the blue dotted and the black dashed lines corresponds to the impact of some 2-loop higher-dimensional operators. The effect of these higher dimensional operators has been discussed in ref. [44], where it has been shown that they are of high relevance for large stop mixing. For small $\left|x_{t}\right| \lesssim 3$ and the shown value $M_{S}=3 \mathrm{TeV}$, however, their effect is negligible. Note, that in figure 5 the hybrid 2-loop result is subtracted from each calculation. Hence, the blue dotted line represents the negative correction due to power suppressed terms. In contrast, figure 4 of ref. [44] shows the positive influence of higher dimensional operators. From the figure we draw the following conclusions:

- The excellent agreement between the black dashed and the blue dotted lines for $\left|x_{t}\right| \lesssim 3$ confirms numerically the correctness of our automatized FlexibleEFTHiggs pole-mass matching procedure for $\hat{\lambda}$ at $\mathcal{O}\left(g_{3}^{2} y_{t}^{4}+y_{t}^{6}\right)$.

- For $\left|x_{t}\right| \gtrsim 3$ the effect of the higher-dimensional 2-loop operators is in line with the numerical results of ref. [44]. 


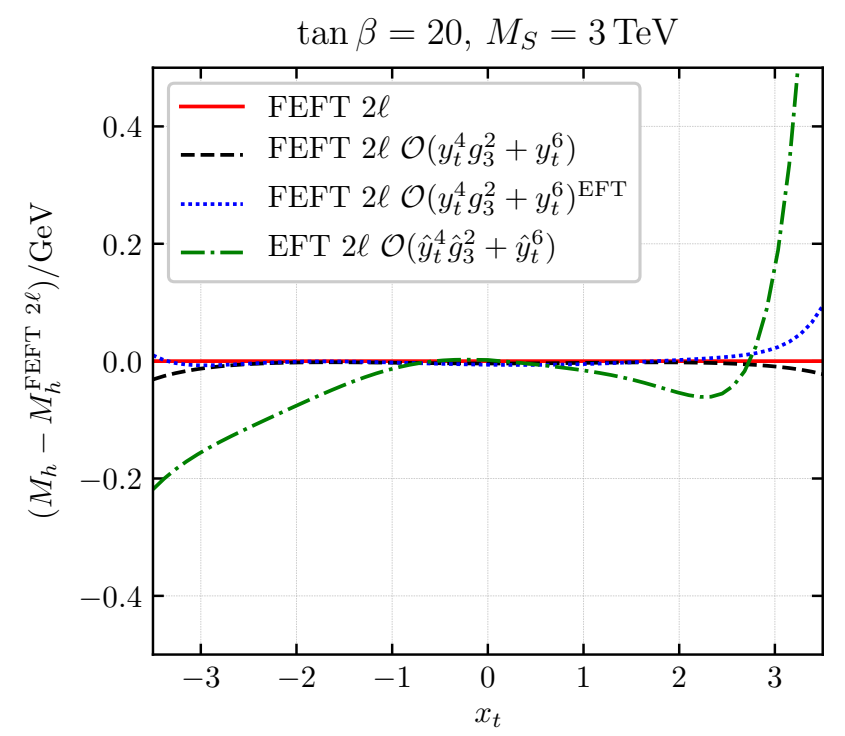

Figure 5. Comparison of our 2-loop hybrid approach (red line and black dashed line) to the 1loop hybrid approach with 2-loop threshold corrections at $\mathcal{O}\left(g_{3}^{2} y_{t}^{4}+y_{t}^{6}\right)$ in the EFT-limit (blue dotted line) and to the pure EFT-calculation HSSUSY with $\Delta \lambda$ included at $\mathcal{O}\left(\hat{g}_{3}^{2} \hat{y}_{t}^{4}+\hat{y}_{t}^{6}\right)$ (green dashed-dotted line).

For reference we also show in figure 5 the EFT $2 \ell$ calculation, represented by the green dashed-dotted line. One finds that EFT $2 \ell$ deviates numerically from FEFT $2 \ell$ for $\left|x_{t}\right| \gtrsim 1$. This discrepancy can be explained by contributions originating from the different parametrization schemes. As motivated above, we categorize the higher-order corrections in two classes of terms; the ones which are incomplete in both approaches and the ones which are captured correctly in our full-model parametrization scheme, but not in the other EFT parametrization.

Concerning the higher-order terms correctly captured by our new FlexibleEFTHiggs hybrid calculation, we find the most dominant contribution to the numerical difference between the EFT $2 \ell$ prediction and FEFT $2 \ell$ to be the 2-loop mixed QCD-EW term from table 3. To illustrate this effect we have created a reparametrized version of the FEFT $2 \ell$ calculation in the EFT parametrization and compare it with EFT $2 \ell$ in figure 6 . The figure shows different 2-loop calculations w.r.t. FEFT $2 \ell$, where at 2-loop level only terms of $\mathcal{O}\left(y_{t}^{4} g_{3}^{2}\right)$ in the EFT-limit $v^{2} \ll M_{S}^{2}$ are taken into account (blue dotted line). The black dashed line represents the reparametrized version of the blue dotted line, where $\hat{\lambda}$ is expressed in terms of SM parameters. In this calculation only 2-loop contributions of $\mathcal{O}\left(\hat{y}_{t}^{4} \hat{g}_{3}^{2}\right)$ are taken into account. One finds that this reparametrized calculation agrees well with the corresponding EFT $2 \ell$ calculation (green dashed-dotted line), which uses the same parametrization. The only difference between the blue dotted and the green dasheddotted line are power suppressed contributions in the Higgs mass at 1-loop, which become significant for $\left|x_{t}\right| \gtrsim 3$, as discussed above. When adding the 2-loop leading $x_{t}$ mixed QCD-EW contribution from eq. (5.11) to the black dashed line, one obtains the red solid line. The so obtained result agrees very well with the MSSM-parametrized calculation 


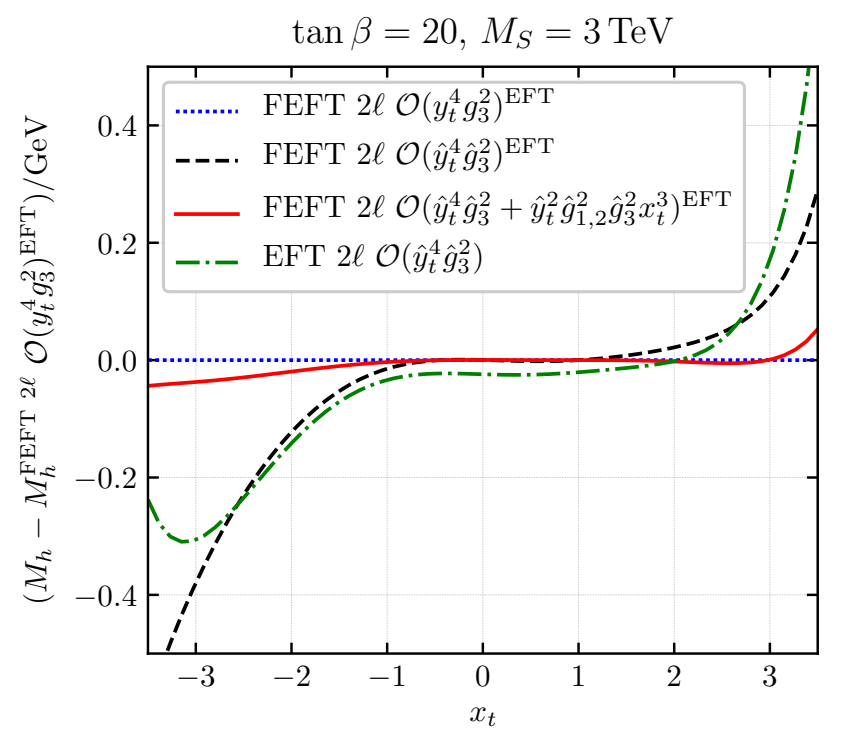

Figure 6. Influence of contributions originating from reparametrization. The plot shows the Higgs pole mass as predicted by different 1-loop calculations with additional 2-loop threshold contributions to $\Delta \lambda$ at $\mathcal{O}\left(y_{t}^{4} g_{3}^{2}\right)$. The superscript "EFT" in the legend indicates that all 2-loop contributions are evaluated in the limit $v^{2} / M_{S}^{2} \rightarrow 0$. The blue dotted line represents the FlexibleEFTHiggs calculation in full-model parametrization. The black dashed line represents the EFT-reparametrized calculation, truncated at $\mathcal{O}\left(\hat{y}_{t}^{4} \hat{g}_{3}^{2}\right)$. The red solid line corresponds to the black dashed line with the additional 2-loop electroweak $x_{t}^{3}$ contribution $\Delta \lambda^{\mathrm{QCD}-\mathrm{EW}}$ from table 3. In the green dashed-dotted line we show the numerical results of the pure EFT calculation including 2-loop corrections to $\Delta \lambda$ in EFT parametrization at $\mathcal{O}\left(\hat{y}_{t}^{4} \hat{g}_{3}^{2}\right)$.

(blue dotted line), which explains the dominant part of the deviation between the MSSMparametrized FEFT $2 \ell$ calculation and the EFT-parametrized EFT $2 \ell$ calculation. Thus, the numerical effect coming from the correct inclusion of highest power $x_{t}$ contributions in our new FlexibleEFTHiggs approach improves the precision for large $\left|x_{t}\right|$ in comparison to the calculation performed in the EFT parametrization.

Besides the higher-order terms correctly taken into account by our new FlexibleEFTHiggs calculation, the threshold corrections $\Delta \lambda$ differ in both approaches by further terms, which are incomplete both in the full-model parametrization and in the EFT parametrization. Such incomplete higher-order terms are for example top Yukawa enhanced 3-loop terms with high $x_{t}$ powers of the form $\left(\hat{\lambda}^{\text {FEFT } 3 \ell}-\hat{\lambda}^{\text {EFT } 3 \ell}\right) \supset \hat{y}_{t}^{8} x_{t}^{\leq 8}+\hat{y}_{t}^{6} \hat{g}_{3}^{2} x_{t}^{\leq 7} \cdot{ }^{20}$ The reparametrization of the 1-loop correction alone was discussed in table 4 . The discussion here is extended by the gauge-less 2-loop contributions to $\Delta \lambda$ in MSSM parametrization. In figure 7 we show the numerical influence of such terms. When these (incomplete) higherorder terms are added coherently (green solid line), both contributions almost cancel up to a remaining effect of $\sim 150 \mathrm{MeV}$ in the Higgs pole mass for $\left|x_{t}\right|<3.5$ and $M_{S}=3 \mathrm{TeV}$. Thus, the numerical effect from the $x_{t}$-resummation terms in $\Delta \lambda^{\mathrm{QCD}-\mathrm{EW}}$ from figure 6

\footnotetext{
${ }^{20}$ Note that in order to investigate the complete reparametrization contributions of this order, the inclusion of 2-loop threshold corrections to $\Delta y_{t}$ at $\mathcal{O}\left(y_{t}^{5}+g_{3}^{2} y_{t}^{3}\right)$ is required.
} 


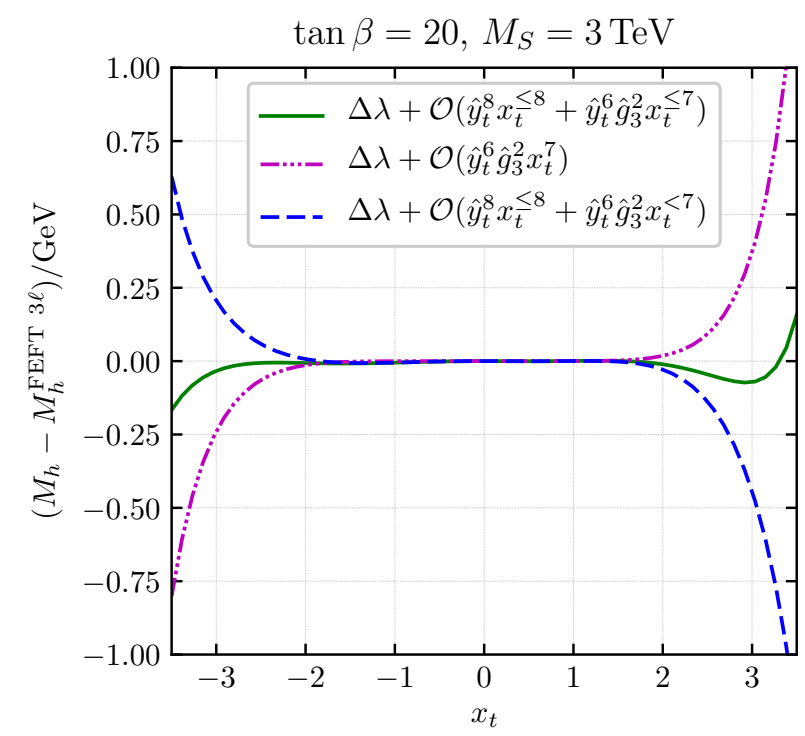

Figure 7. Impact of incomplete higher-order contributions to $\Delta \lambda$ from reparametrization in FlexibleEFTHiggs.

remains the dominant reparametrization effect. However, when the numerical effect of each incomplete higher-order term is drawn individually, the contributions have a higher impact on the Higgs pole mass, see figure 7. The magenta dashed-triple-dotted line corresponds to the effect of the terms of $\mathcal{O}\left(\hat{y}_{t}^{6} \hat{g}_{3}^{2} x_{t}^{7}\right)$ and the blue dashed line corresponds to $\mathcal{O}\left(\hat{y}_{t}^{8} x_{t}^{\leq 8}+\hat{y}_{t}^{6} \hat{g}_{3}^{2} x_{t}^{<7}\right)$. There is a cancellation between these incomplete contributions, which should be kept in mind when using such terms as an uncertainty estimate of missing higher-order corrections. Using the maximum effect of all terms provides a more conservative estimate of the remaining uncertainty than the coherent sum.

\section{Uncertainty estimation}

In this section we analyze missing higher-order contributions in our new FlexibleEFTHiggs approach in order to estimate the remaining theory uncertainty of our calculation. In accordance with refs. [41, 42, 47] we distinguish between missing higher-order contributions in the matching at the SUSY scale, which we denote as high-scale uncertainty, and missing loop corrections at the electroweak scale, denoted as low-scale uncertainty. Note, that since FlexibleEFTHiggs is a hybrid calculation, we do not assign an EFT uncertainty to our calculation from missing terms of $\mathcal{O}\left(v^{2} / M_{S}^{2}\right) \cdot{ }^{21}$

\subsection{High-scale uncertainty}

We begin our discussion by presenting our methods to estimate the high-scale uncertainty, i.e. the numerical impact of the missing higher-order corrections in the matching of the

\footnotetext{
${ }^{21}$ Our calculation of the Higgs mass does not include suppressed logarithms beyond the 2-loop gaugeless limit. In fact, in ref. [44] it has been demonstrated that their impact is very small $\Delta M_{h} \leq 20 \mathrm{MeV}$ for the studied scenarios.
} 
MSSM $\overline{\mathrm{DR}}^{\prime}$ to the SM $\overline{\mathrm{MS}}$ parameters at the SUSY scale. We discuss three different approaches: the variation of the matching scale, implicit higher-order corrections from the double loop expansion and reparametrization terms.

Variation of the matching scale. A commonly applied strategy to estimate higherorder contributions is to vary the renormalization scale $Q_{\text {match }}$ at which the threshold corrections are computed. For reasons of comparability, we use the conventional range of $Q_{\text {match }} \in\left[M_{S} / 2,2 M_{S}\right]$ and take the maximum deviation from the value obtained at $Q_{\text {match }}=M_{S}$ as an estimate

$$
\Delta M_{h}^{Q_{\text {match }}}=\max _{Q \in\left[M_{S} / 2,2 M_{S}\right]}\left\{\left|M_{h}\left(Q_{\text {match }}=M_{S}\right)-M_{h}\left(Q_{\text {match }}=Q\right)\right|\right\} .
$$

The numerical variation of $M_{h}$ results from the fact that the matching corrections contain explicit dependencies of $\log Q^{2}$ at fixed order, while the RGE running cancels those logarithms but also generates $\log Q^{2}$ terms at higher orders. The quantity $\Delta M_{h}^{Q_{\text {match }}}$ thus represents an estimate for these missing logarithmic higher-order terms. In particular, in the matching of $\hat{\lambda}$, the following 2-loop and 3-loop terms are generated:

$$
\Delta \lambda_{h}^{Q_{\text {match }}} \supset \propto y_{t}^{2}\left(g_{3}^{2} g_{1,2}^{2}+y_{t}^{2} g_{1,2}^{2}+g_{1,2}^{4}+y_{t}^{2} g_{3}^{4} x_{t}^{4}+y_{t}^{4} g_{3}^{2}+y_{t}^{6}\right)+\mathcal{O}\left(g_{1,2}^{6}\right) .
$$

The matching-scale variation thus provides an estimate of the theory uncertainty related to these terms, at least to their $\log Q^{2}$-dependent parts. We have omitted the specification of the powers of $x_{t}$ in most of the terms. The term of $\mathcal{O}\left(y_{t}^{4} g_{3}^{4} x_{t}^{4}\right)$ deserves special attention: in the degenerate mass case the Himalaya library up to version 3.0.1 does not provide the correct term in the Higgs mass correction at this order [33]. Since this is an important missing term of higher order in $x_{t}$, but not of higher order in the couplings, we have verified that this missing term of this order has a non-vanishing $\log Q^{2}$ dependence. Indeed, employing 2-loop $\beta$ functions from ref. [96] on the 2-loop Higgs pole mass, derived from the effective potential of ref. [97], the renormalization scale dependence in the degenerate mass case is given by

$$
\frac{\partial}{\partial \log Q^{2}} \Delta s_{h}^{\mathrm{MSSM}, y_{t}^{4} g_{3}^{4}}=\frac{g_{3}^{4} y_{t}^{4} v^{2}}{(4 \pi)^{6}} \frac{224}{9}\left(x_{t}^{4}+\propto x_{t}^{\leq 3}\right) .
$$

Thus, the matching scale variation in our calculation provides an estimate of the uncertainty originating from missing logarithmic terms at $\mathcal{O}\left(y_{t}^{4} g_{3}^{4} x_{t}^{4}\right)$ in particular.

We'd like to point out a technical difficulty in this matching scale variation. The evolution of RGEs in the MSSM requires the numerical input values of MSSM $\overline{\mathrm{DR}}^{\prime}$ parameters as a boundary condition. However, in the MSSM two parameters cannot be fixed by the input; rather they have to be eliminated by imposing the two electroweak symmetry breaking conditions. Solving these so-called tadpole equations at the loop level will introduce logarithms which contain light masses. Hence, it is a legitimate question to ask whether such contributions spoil the automatized cancellation of large logarithms in the matching correction. In our calculation, the tadpole equations at the SUSY scale are solved for the dimensionful soft-breaking Higgs-doublet mass parameters $m_{H_{u}}^{2}$ and $m_{H_{d}}^{2}$. An explicit calculation up to leading 2-loop QCD order shows that large logarithms enter into $\hat{\lambda}$ with a 
suppression of $v^{2} / M_{S}^{2}$ beyond the considered order. These contributions would be absent in a pure EFT calculation and they can be regarded as a power-suppressed contribution in a hybrid calculation.

Implicit corrections at higher order. In section 4 we discussed the expansion of the master formula (4.8) and explained how "explicit" contributions from genuine multi-loop diagrams are accompanied by "implicit" corrections in the double loop expansion, i.e. from the reparametrization of the SM self-energy in terms of MSSM parameters. These implicit corrections have the form of products of derivatives of the SM Higgs pole mass shift $\Delta s_{h}^{\mathrm{SM}}$ times parameter shifts.

Hence, as another estimate of missing higher-order corrections, we compute further terms with such a structure at orders beyond the precision of the included threshold corrections and discard terms which contain logarithms of the form $\log \left(m_{t} / Q\right)$. The resulting contributions take the form

$$
\frac{1}{\hat{v}^{2}}\left[\sum_{P}\left(\frac{\partial}{\partial P} \Delta s_{h}^{\mathrm{SM}}\right) \Delta P\right]_{\log \left(m_{t} / Q\right)=0}=\Delta \lambda_{g_{1}, g_{2}}^{2 \ell}+\Delta \lambda_{g_{3}, y_{t}}^{3 \ell},
$$

where $\Delta \lambda_{g_{1}, g_{2}}^{2 \ell}$ denotes terms which would arise in an actual 2-loop calculation beyond the gaugeless limit, and $\Delta \lambda_{g_{3}, y_{t}}^{3 \ell}$ contains terms which would arise in an actual 3-loop calculation in the gaugeless limit. The corresponding orders in couplings are

$$
\begin{aligned}
\Delta \lambda_{g_{1}, g_{2}}^{2 \ell} \supset & \propto g_{1,2}^{2}\left[g_{3}^{2} y_{t}^{2} x_{t}^{\leq 1}+y_{t}^{4} x_{t}^{\leq 4}+y_{t}^{2} g_{1,2}^{2} x_{t}^{\leq 2}+g_{1,2}^{4}\right], \\
\Delta \lambda_{g_{3}, y_{t}}^{3 \ell} & \equiv \Delta \lambda_{g_{3}^{4} y_{t}^{4} x_{t}^{\leq 2}}^{3 \ell}+\Delta \lambda_{g_{3}^{2} y_{t}^{6} x_{t}^{\leq 5}, y_{t}^{8} x_{t}^{\leq 8}} .
\end{aligned}
$$

The 3-loop gaugeless contributions contained in the generated terms on the r.h.s. of eq. (8.6) are of the order as indicated in the subscript.

We can thus first define an estimate of the size of the missing 2-loop electroweak SUSY corrections as

$$
\Delta M_{h}^{\mathrm{imp}, g_{1,2}, 2 \ell}=\left|M_{h}\left(\Delta \lambda^{3 \ell}\right)-M_{h}\left(\Delta \lambda^{3 \ell}+\Delta \lambda_{g_{1}, g_{2}}^{2 \ell}\right)\right|,
$$

where $M_{h}\left(\Delta \lambda^{3 \ell}\right)$ denotes the FEFT $3 \ell$ calculation. Next, we can define an estimate of the size of missing higher-order SUSY-QCD contributions as

$$
\begin{aligned}
& \Delta M_{h}^{\mathrm{imp}, g_{3} y_{t}, 2 \ell}=\mid M_{h}\left(\Delta \lambda^{2 \ell}\right)-M_{h}\left(\Delta \lambda^{2 \ell}+\Delta \lambda_{g_{3}^{4} y_{t}^{4} x_{t}^{\leq 2}}^{3 \ell}+\Delta \lambda_{g_{3}^{2} y_{t}^{6} x_{t}^{\leq 5}, y_{t}^{8} x_{t}^{\leq 8}}^{3 \ell} \mid,\right. \\
& \Delta M_{h}^{\mathrm{imp}, g_{3} y_{t}, 3 \ell}=\mid M_{h}\left(\Delta \lambda^{3 \ell}\right)-M_{h}\left(\Delta \lambda^{3 \ell}+\Delta \lambda_{\left.g_{3}^{2} y_{t}^{6} x_{t}^{\leq 5}, y_{t}^{8} x_{t}^{\leq 8}\right) \mid}\right.
\end{aligned}
$$

of the FEFT $2 \ell$ and $3 \ell$ calculations, respectively. Note, that for the uncertainty estimate of the FEFT $3 \ell$ calculation (8.8b), we do not use the 3-loop terms $\Delta \lambda_{g_{3}^{4} y_{t}^{4} x_{t}^{\leq 2}}^{3 \ell}$, since they are already included in the known 3-loop threshold corrections $\Delta \lambda^{3 \ell}$ at $\mathcal{O}\left(y_{t}^{4} g_{3}^{4}\right)$.

Note, that since the derivatives of the SM self-energy do not depend on the MSSM parameters, the $x_{t}$ dependence of the terms contained in eq. (8.4) is only introduced by the shift $\Delta P$. This is the reason for the particular maximum powers of $x_{t}$ which appear 
in eqs. (8.5) and (8.6). In particular, at the order $g_{3}^{2} y_{t}^{6}$, these uncertainty estimates only contain terms up to $x_{t}^{5}$, while the true threshold correction at this order is allowed to contain $x_{t}^{6}$. Hence the method of implicit corrections cannot reliably estimate the influence of the highest-power $x_{t}$ contributions.

Reparametrization terms. For the reasons discussed in the previous sections, we chose to express the threshold corrections in terms of MSSM parameters. When computed at all orders in perturbation theory, both the full-model and the EFT parametrization do not differ by definition. Hence, it is possible to estimate the uncertainty of missing higher-order contributions by the numerical difference of the Higgs mass prediction in both parametrizations. The full-model parametrization is preferred because at some finite order in MSSM parameters it already resums highest power $x_{t}$ corrections of QCD-enhanced orders in SM parameters. However, we can use reparametrization to generate terms of orders which are missing or incomplete in our calculation. Specifically, already in section 7.3, in the context of figure 7, such reparametrization terms of the orders

$$
\Delta \lambda^{\mathrm{rep}} \supset \hat{y}_{t}^{8} x_{t}^{\leq 8}+\hat{y}_{t}^{6} \hat{g}_{3}^{2} x_{t}^{\leq 7}
$$

were discussed. In contrast to the implicit corrections, reparametrization generates terms of highest order in $x_{t}$ which can appear in the true threshold correction, and the reparametrization terms in eq. (8.9) can thus more reliably estimate the influence of missing highest-power $x_{t}$ contributions. Because of the nature of reparametrization, this method also estimates missing higher-order terms in the threshold corrections $\Delta y_{t}$ and $\Delta g_{3}$. For later discussion of the size of the reparametrization terms of eq. (8.9), we define the following uncertainty estimates,

$$
\begin{gathered}
\Delta M_{h}^{\mathrm{rep}, g_{3}, 3 \ell}=\left|M_{h}\left(\Delta \lambda^{3 \ell}\right)-M_{h}\left(\Delta \lambda^{3 \ell}-\mathcal{O}\left(\hat{y}_{t}^{6} \hat{g}_{3}^{2} x_{t}^{7}\right)\right)\right|, \\
\Delta M_{h}^{\mathrm{rep}, g_{3} y_{t}, 3 \ell}=\left|M_{h}\left(\Delta \lambda^{3 \ell}\right)-M_{h}\left(\Delta \lambda^{3 \ell}-\mathcal{O}\left(\hat{y}_{t}^{6} \hat{g}_{3}^{2} x_{t}^{<7}+\hat{y}_{t}^{8} x_{t}^{\leq 8}\right)\right)\right|,
\end{gathered}
$$

where we subtract the reparametrization terms from the FEFT $3 \ell$ calculation in order to reproduce the truncation of the EFT parametrization of $\hat{\lambda}$ at $\mathcal{O}\left(1 \ell+\hat{g}_{3}^{2}\left(\hat{y}_{t}^{4}+\hat{y}_{b}^{4}\right)+\left(\hat{y}_{t}^{2}+\right.\right.$ $\left.\left.\hat{y}_{b}^{2}\right)^{3}+\left(\hat{y}_{t}^{2}+\hat{y}_{\tau}^{2}\right)^{3}+\hat{g}_{3}^{4} \hat{y}_{t}^{4}\right)$. Up to a sign, the dashed-triple-dotted magenta line and the dashed blue line in figure 7 shows equivalently the numerical influence of the terms estimated by $\Delta M_{h}^{\mathrm{rep}, g_{3}, 3 \ell}$ and $\Delta M_{h}^{\mathrm{rep}, g_{3} y_{t}, 3 \ell}$.

At this point it is worthwhile to discuss the difference in the estimation of the uncertainty of an EFT-parametrized calculation at similar order, i.e. with a matching of $\hat{\lambda}$ at $\mathcal{O}\left(1 \ell+\hat{g}_{3}^{2}\left(\hat{y}_{t}^{4}+\hat{y}_{b}^{4}\right)+\left(\hat{y}_{t}^{2}+\hat{y}_{b}^{2}+y_{\tau}^{2}\right)^{3}+\hat{g}_{3}^{4} \hat{y}_{t}^{4}\right)$. The reparametrization provides a way to estimate higher-order terms in this calculation, which are sensitive to high powers of $x_{t}$. Furthermore, the uncertainty estimation should also cover terms of $\mathcal{O}\left(\hat{y}_{t}^{8}, \hat{y}_{t}^{6} \hat{g}_{3}^{2}\right)$, which are incomplete in both parametrizations, cf. table 4. Consequently, if the discussed techniques are applied to construct higher-order terms for the uncertainty estimation of the EFT-parametrized calculation, we expect that they lead to very similar expressions for $\Delta \lambda \supset \hat{y}_{t}^{8} x_{t}^{\leq 8}+\hat{y}_{t}^{6} \hat{g}_{3}^{2} x_{t}^{\leq 7}$. Note, that in contrast to the full-model-parametrized calculation, the EFT-parametrized one would in addition have to estimate the size of the terms of 
$\Delta \lambda \supset \hat{y}_{t}^{2} \hat{g}_{1}^{2} \hat{g}_{3}^{2} x_{t}^{3}+\hat{y}_{t}^{2} \hat{g}_{2}^{2} \hat{g}_{3}^{2} x_{t}^{3}+\hat{y}_{t}^{4} \hat{g}_{3}^{6} x_{t}^{7}$, which are implicitly captured in full-model parametrization. Thus, in EFT parametrization more higher order contributions would be needed to estimate the uncertainty for large $\left|x_{t}\right|$.

\subsection{Low-scale uncertainty}

In this section we describe our method to estimate the low-scale uncertainty, i.e. the theory uncertainty from missing higher-order loop corrections in the matching to the SM input parameters at the electroweak scale. We consider two different approaches: the variation of the renormalization scale of the Higgs pole mass calculation and the variation of loop orders in the determination of the top Yukawa coupling.

Variation of the pole mass scale. First we discuss the variation of the renormalization scale at which the pole mass $M_{h}$ is computed in the SM. By default the scale $Q_{\text {pole }}=M_{t}$ is chosen, which we vary by factor of two,

$$
\Delta M_{h}^{Q_{\text {pole }}}=\max _{Q \in\left[M_{t} / 2,2 M_{t}\right]}\left\{\left|M_{h}\left(Q_{\text {pole }}=M_{t}\right)-M_{h}\left(Q_{\text {pole }}=Q\right)\right|\right\} .
$$

This procedure estimates the impact of missing logarithmic higher-order corrections to the Higgs pole mass shift in the SM.

Variation of the loop order of threshold corrections at the low scale. As described in section 6 , the relation between low-energy observables and $\overline{\mathrm{MS}}$-renormalized SM couplings contains corrections that can be switched off in the calculation without reducing the precision of the result for $M_{h}$. As was shown in refs. [41, 42, 47,61], the dominant uncertainty obtained from this procedure is driven by the higher-order threshold correction in the relation between the top quark pole mass and the top Yukawa coupling. We define our estimation of missing threshold corrections at the electroweak scale in accordance with that reference as

$$
\begin{aligned}
& \Delta M_{h}^{y_{t}, 2 \ell}=\left|M_{h}^{y_{t}, 2 \ell}-M_{h}^{y_{t}, 3 \ell}\right|, \\
& \Delta M_{h}^{y_{t}, 3 \ell}=\left|M_{h}^{y_{t}, 3 \ell}-M_{h}^{y_{t}, 4 \ell}\right|,
\end{aligned}
$$

where the superscript of the symbols $M_{h}^{y t, n \ell}$ indicates that eq. (6.5) is evaluated at $n$-loop level. Since the consistent resummation of NNLL/ $\mathrm{N}^{3} \mathrm{LL}$ logarithms requires an evaluation of eq. (6.5) at 2-/3-loop level, we estimate the uncertainty of the FEFT $2 \ell / 3 \ell$ calculation by $\Delta M_{h}^{y_{t}, 2 \ell}$ and $\Delta M_{h}^{y_{t}, 3 \ell}$, respectively.

\subsection{Numerical size of individual uncertainties}

In figure 8 we show the individual sizes of the uncertainty estimates discussed above for the parameter scenarios from figure 4 . The two black lines correspond to uncertainties for FEFT $2 \ell$ and the other lines correspond to FEFT $3 \ell$.

\subsubsection{High-scale uncertainty}

We start with a discussion of the high-scale uncertainty, shown in the left column of figure 8. 

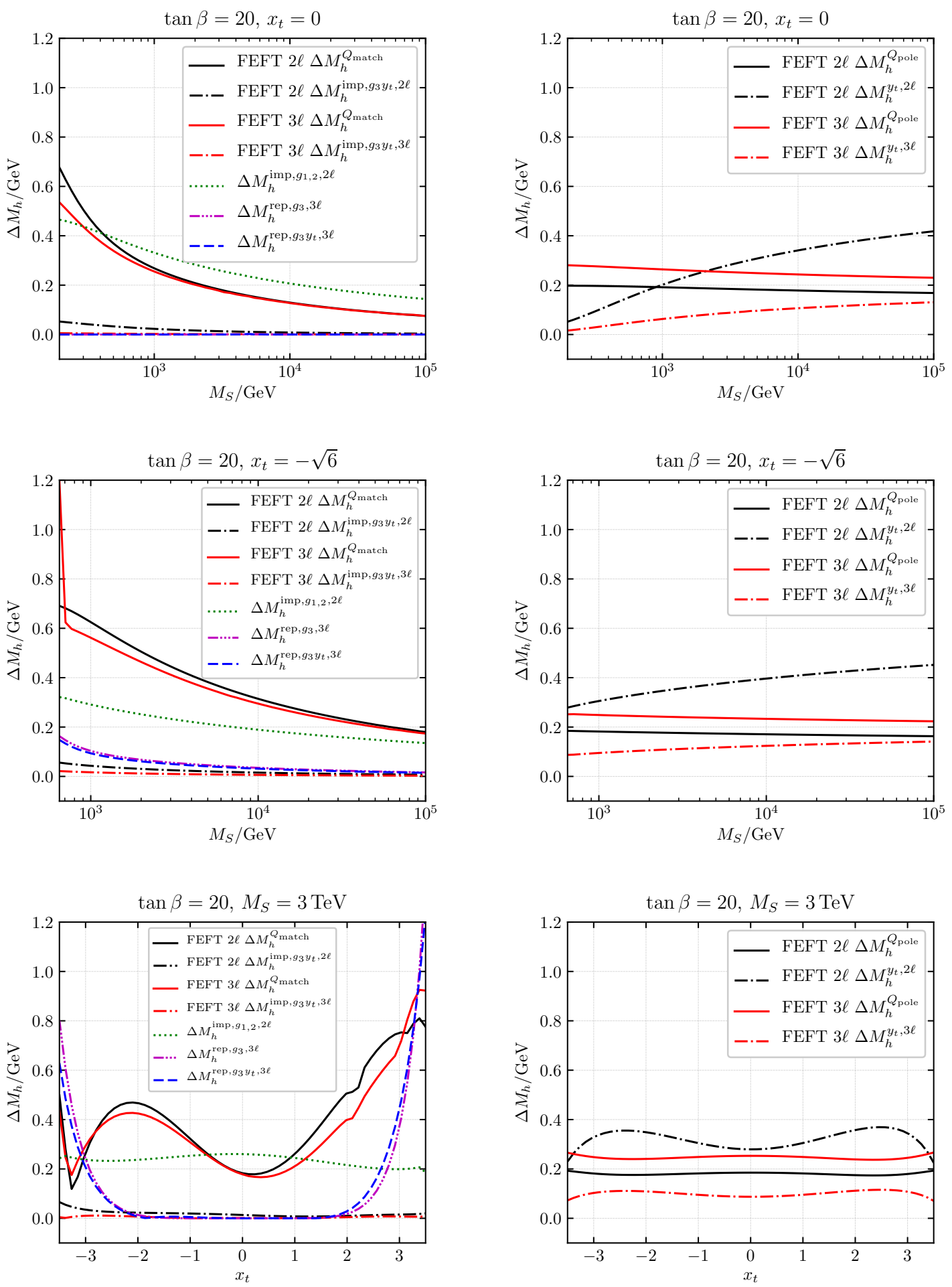

Figure 8. Individual contributions to the high-scale uncertainty (left column) and to the low-scale uncertainty (right column). 
Estimate of missing 3-loop QCD and $y_{t}$-enhanced contributions beyond $\mathcal{O}\left(\boldsymbol{y}_{t}^{4} g_{3}^{4} x_{t}^{\leq 4}\right)$. In figure 8, the black and red solid lines represent the matching-scale uncertainties $\Delta M_{h}^{Q_{\text {match }}}$ of the 2- and 3-loop FEFT calculation, respectively. The matching-scale uncertainty provides a global estimate of many kinds of terms, see eq. (8.2). The difference between the black and red solid lines corresponds to the inclusion of the known leadingQCD 3-loop contributions of $\mathcal{O}\left(y_{t}^{4} g_{3}^{4} x_{t}^{\leq 3}\right)$ to $\Delta \lambda$. We find that this inclusion reduces the uncertainty very little, less than $0.2 \mathrm{GeV}$ for all studied scenarios. In particular, since terms of the order $\mathcal{O}\left(y_{t}^{4} g_{3}^{4} x_{t}^{4}\right)$ are not known for all parameter scenarios, we expect a remaining uncertainty of significant size for large $\left|x_{t}\right|$ (see lower left panel of figure 8). Note, that $\Delta M_{h}^{Q_{\text {match }}}$ is sensitive to terms of $\mathcal{O}\left(y_{t}^{4} g_{3}^{4} x_{t}^{4}\right)$ (cf. eq. (8.3)) and thus includes an estimate of these missing terms.

To provide a direct estimate the size of the missing non-logarithmic 3-loop QCD and $y_{t}$-enhanced contributions, we show as black and red dashed-dotted lines the uncertainties $\Delta M_{h}^{\text {imp }, g_{3} y_{t}, 2 \ell}$ and $\Delta M_{h}^{\text {imp, } g_{3} y_{t}, 3 \ell}$ for the 2- and 3-loop FEFT calculations, respectively. We find that these QCD uncertainties are very small already for the 2-loop calculation, $\Delta M_{h}^{\mathrm{imp}, g_{3} y_{t}, 2 \ell} \lesssim 0.1 \mathrm{GeV}$. This is fully in line with the small difference between the 2-loop and 3-loop matching-scale uncertainty described above. The 3-loop QCD and $y_{t}$-enhanced corrections missing in FEFT $3 \ell, \Delta M_{h}^{\mathrm{imp}, g_{3} y_{t}, 3 \ell}$, including terms with fewer powers of $g_{3}$, are found to be negligible (red dashed-dotted line).

Taken together, all these results provide strong evidence that the contributions of leading QCD-type are already very well under control and inclusion of higher-order leadingQCD threshold corrections of $\mathcal{O}\left(y_{t}^{4} g_{3}^{6}\right)$ will not improve the precision of the calculation significantly.

Importance of 2-loop electroweak contributions. The size of the missing 2-loop electroweak SUSY contributions to $\Delta \lambda$ are estimated by the matching-scale variation, $\Delta M_{h}^{Q_{\text {match }}}$, and more directly by the generated implicit contributions, $\Delta M_{h}^{\text {imp }, g_{1,2}, 2 \ell}$, defined in section 8.1. The implicit contributions, shown as green dotted line in figure 8, have a sizable numerical effect of $\Delta M_{h}^{\mathrm{imp}, g_{1,2}, 2 \ell} \lesssim 0.4 \mathrm{GeV}$. Further investigations of the induced terms from eq. (8.5) indicate that the terms of $\mathcal{O}\left(y_{t}^{4} g_{1,2}^{2}\right)$ are typically dominant for the parameter scenarios studied here. For vanishing stop mixing, $\Delta M_{h}^{\mathrm{imp}, g_{1,2}, 2 \ell}$ is of the same order as $\Delta M_{h}^{Q_{\text {match }}}$. More precisely, the offset of the solid lines, i.e. the smallest value of $\Delta M_{h}^{Q_{\text {match }}}$ at $x_{t} \approx-3$, in the lower left panel of figure 8 is of the same magnitude as the almost constant green dotted line. For $x_{t}=0$ and $M_{S}>1 \mathrm{TeV}$, both $\Delta M_{h}^{Q_{\text {match }}}$ and $\Delta M_{h}^{\mathrm{imp}, g_{1,2}, 2 \ell}$ predict that the uncertainty decreases at the same rate when going to higher $M_{S} \cdot{ }^{22}$

This indicates that missing electroweak 2-loop terms contribute a theory uncertainty which is typically around $0.2-0.3 \mathrm{GeV}$, has a weak $x_{t}$-dependence, and which is the dominant theory uncertainty for small $\left|x_{t}\right|$.

\footnotetext{
${ }^{22}$ The middle-left plot in figure 8 shows a numerical instability at $M_{S}<750 \mathrm{GeV}$ in the FEFT $3 \ell$ calculation due to a hierarchy switch in Himalaya. The kink in the curves for $\Delta M_{h}^{Q_{\text {match }}}$ in the lower left panel at $x_{t} \approx-3.2$ is due to a numerical artifact of our definition of the uncertainty. The irregularities at $x_{t} \approx 2$ in the middle-left plot are due to a numerical instability in our code, which is absent for lower values of $\tan \beta$.
} 
Relevant higher-order contributions for large $\left|\boldsymbol{x}_{\boldsymbol{t}}\right|$. For large $\left|x_{t}\right| \gtrsim 2$ the matchingscale uncertainty $\Delta M_{h}^{Q_{\text {match }}}$ is larger than for small $x_{t}$. This cannot be attributed exclusively to the missing leading-QCD and 2-loop electroweak terms discussed so far. As discussed in section 8.1 and ref. [54], this is not unexpected because of the low powers of $x_{t}$ appearing in $\Delta M_{h}^{\mathrm{imp}, g_{3} y_{t}, 3 \ell}$. On the other hand, the increased uncertainty for large $x_{t}$ is in line with the discussion of the impact of non-resummed large- $x_{t}$ contributions in section 7.3. In order to estimate missing terms with high $x_{t}$-dependence, we employ the uncertainty estimates based on reparametrization terms. Indeed, reparametrization terms $\Delta M_{h}^{\mathrm{rep}, g_{3}, 3 \ell}$ (magenta dashed-triple-dotted line) and $\Delta M_{h}^{\mathrm{rep}, g_{3} y_{t}, 3 \ell}$ (blue dashed line) in figure 8 do contain the maximal powers of $x_{t}$ at their respective loop order. In fact, the combinations $\Delta M_{h}^{\mathrm{imp}, g_{1,2}, 2 \ell}+\Delta M_{h}^{\mathrm{rep}, g_{3}, 3 \ell}$ and $\Delta M_{h}^{\mathrm{imp}, g_{1,2}, 2 \ell}+\Delta M_{h}^{\mathrm{rep}, g_{3} y_{t}, 3 \ell}$ are of the order of $\Delta M_{h}^{Q_{\text {match }}}$, see the middle panel in the left column of figure 8. This suggests that both electroweak and QCD and $y_{t}$-enhanced terms with high powers of $x_{t}$ are the dominant source of uncertainty for large stop mixing, which must be brought under control to reduce the high-scale uncertainty further.

For $\left|x_{t}\right| \approx 3$, the uncertainty estimate obtained from reparametrization becomes dominant. However, this is not specific to performing the calculation in full-model parametrization and it cannot be interpreted as an indication that the EFT parametrization would perform better with regard to missing contributions at $\mathcal{O}\left(\hat{y}_{t}^{8}+\hat{y}_{t}^{6} \hat{g}_{3}^{2}\right)$. In fact, as discussed at the end of section 8.1, the estimation of the uncertainty for a calculation performed in SM parametrization would lead to a similar result at these orders.

\subsubsection{Low-scale uncertainty}

Now we discuss the size of the low-scale uncertainty as defined by the measures in section 8.2. The individual sources of the low-scale uncertainty are shown in the right column of figure 8 . The variation of the pole mass scale, $\Delta M_{h}^{Q_{\text {pole }}}$, is shown by the solid lines for FEFT $2 \ell$ (black solid line) and FEFT $3 \ell$ (red solid line). We find excellent agreement of the pole mass uncertainty of FEFT $2 \ell$ with the corresponding result shown in figure 3 of ref. [47]. Concerning the FEFT $3 \ell$ calculation we find a larger uncertainty of $\Delta M_{h}^{Q_{\text {pole }}}$ than the corresponding FEFT $2 \ell$ calculation, which is surprising at first sight. The reason for this is the inclusion of the 3-loop Higgs pole mass shift in the SM of $\mathcal{O}\left(\hat{v}^{2} \hat{y}_{t}^{4} \hat{g}_{3}^{4}\right)$ from ref. [70], which has the particular property that it increases the sensitivity of the Higgs pole mass on renormalization scale, if the scale is varied within $Q_{\text {pole }} \in\left[M_{t} / 2,2 M_{t}\right]$. However, if the scale $Q_{\text {pole }}$ is varied within a larger range, the inclusion of this 3-loop correction leads to a significantly reduced dependence of the Higgs pole mass on $Q_{\text {pole }}$. In order to keep our results comparable with the literature, we stick to the convention of using the $Q_{\text {pole }} \in\left[M_{t} / 2,2 M_{t}\right]$. As a result, we find $\Delta M_{h}^{Q_{\text {pole }}} \lesssim 0.3 \mathrm{GeV}$ for FEFT $3 \ell$ in the shown parameter scenarios.

Our second measure to estimate part of the low-scale uncertainty is given by the influence of higher-order correction in the relation between the Yukawa coupling and the pole mass of the top quark, $\Delta M_{h}^{y_{t}, n \ell}$, defined in eqs. (8.12). The uncertainties $\Delta M_{h}^{y_{t}, 2 \ell}$ and $\Delta M_{h}^{y_{t}}, 3 \ell$ of the FEFT $2 \ell$ and $3 \ell$ calculations are shown as black and red dashed-dotted lines in the right column of figure 8 , respectively. Again, by comparing the uncertainty 
$\Delta M_{h}^{y_{t}, 2 \ell}$ with the corresponding result from figure 3 of ref. [47], we find excellent agreement. Compared to the FEFT $2 \ell$ calculation, the FEFT $3 \ell$ calculation has a strong reduction of the uncertainty with $\Delta M_{h}^{y_{t}, 3 \ell} \lesssim 0.2 \mathrm{GeV}$. This is the main source of the improved precision of our 3-loop calculation of $M_{h}$ in the studied scenarios.

\subsection{Combined uncertainty}

In this subsection we combine the individual uncertainty estimates presented in the previous subsections to obtain a total uncertainty estimate of our new 2-loop and 3-loop FlexibleEFTHiggs calculations. Since the individual uncertainty estimates at the high- and lowenergy scales are sensitive to an overlap of higher-order terms, we define the following combined high-scale uncertainty, $\Delta M_{h}^{\mathrm{HS}}$, and low-scale uncertainty, $\Delta M_{h}^{\mathrm{LS}}$, for the FEFT $n \ell$ calculation:

$$
\begin{aligned}
& \Delta M_{h}^{\mathrm{HS}}=\max \left\{\Delta M_{h}^{Q_{\text {match }}}, \Delta M_{h}^{\lambda, n \ell}\right\} \\
& \Delta M_{h}^{\mathrm{LS}}=\max \left\{\Delta M_{h}^{Q_{\text {pole }}}, \Delta M_{h}^{y_{t}, n \ell}\right\} .
\end{aligned}
$$

In eq. (8.13a), $\Delta M_{h}^{\lambda, n \ell}$ refers to the following combination of our different approaches of generating higher-order terms in $\hat{\lambda}$ as described in section 8.1,

$$
\Delta M_{h}^{\lambda, n \ell}=\Delta M_{h}^{\mathrm{imp}, g_{1,2}, 2 \ell}+\max \left\{\Delta M_{h}^{\mathrm{imp}, g_{3} y_{t}, n \ell}, \Delta M_{h}^{\mathrm{rep}, g_{3} y_{t}, 3 \ell}, \Delta M_{h}^{\mathrm{rep}, g_{3}, 3 \ell}\right\} .
$$

Since the uncertainty estimates $\Delta M_{h}^{\mathrm{imp}, g_{3} y_{t}, n \ell}, \Delta M_{h}^{\mathrm{rep}, g_{3} y_{t}, 3 \ell}$ and $\Delta M_{h}^{\mathrm{rep}, g_{3}, 3 \ell}$ are sensitive to an overlap of higher-order contributions to $\hat{\lambda}$ that involve terms of $\mathcal{O}\left(y_{t}^{n} g_{3}^{m}\right)$, we take their maximum in eq. (8.14). On the other hand, the electroweak contributions $\Delta M_{h}^{\mathrm{imp}, g_{1,2}, 2 \ell}$ are an independent subset of higher-order terms that involve electroweak gauge couplings, so we add it linearly to the other terms in eq. (8.14). To obtain the total combined uncertainty, $\Delta M_{h}$, of our calculations, we add the high-scale and low-scale uncertainties linearly,

$$
\Delta M_{h}=\Delta M_{h}^{\mathrm{HS}}+\Delta M_{h}^{\mathrm{LS}} .
$$

For the degenerate SUSY mass scenarios defined in section 7 , the results of our combined uncertainty estimates are shown in figure 9 . The red solid line represents the Higgs pole mass $M_{h}$ obtained with the FEFT $3 \ell$ calculation and the red band in the lower sub-plots denotes the corresponding combined uncertainty $\Delta M_{h}$. The black dashed lines correspond to the FEFT $2 \ell$ calculation accordingly. The difference between the FEFT $3 \ell$ and $2 \ell$ calculations is of the order $\left|M_{h}^{3 \ell}-M_{h}^{2 \ell}\right| \lesssim 0.3 \mathrm{GeV}$. Compared to the 2-loop calculation, we find a more pronounced decrease of the uncertainty of the 3-loop calculation for large stop mixing $\left|x_{t}\right| \sim 2$ and $M_{S} \gtrsim 5 \mathrm{TeV}$. The dominant reduction of the total uncertainty of the 3-loop calculation is achieved in the low-scale uncertainty $\Delta M_{h}^{\mathrm{LS}}$, where $\Delta M_{h}^{y_{t}, 2 \ell}$ is the dominant uncertainty of the 2-loop calculation.

In general we find for the studied degenerate $\overline{\mathrm{DR}}^{\prime}$ SUSY mass parameter scenarios a combined uncertainty of the FEFT $3 \ell$ calculation of $\Delta M_{h} \lesssim 1 \mathrm{GeV}$ for $M_{S} \gtrsim 1 \mathrm{TeV}$ and 

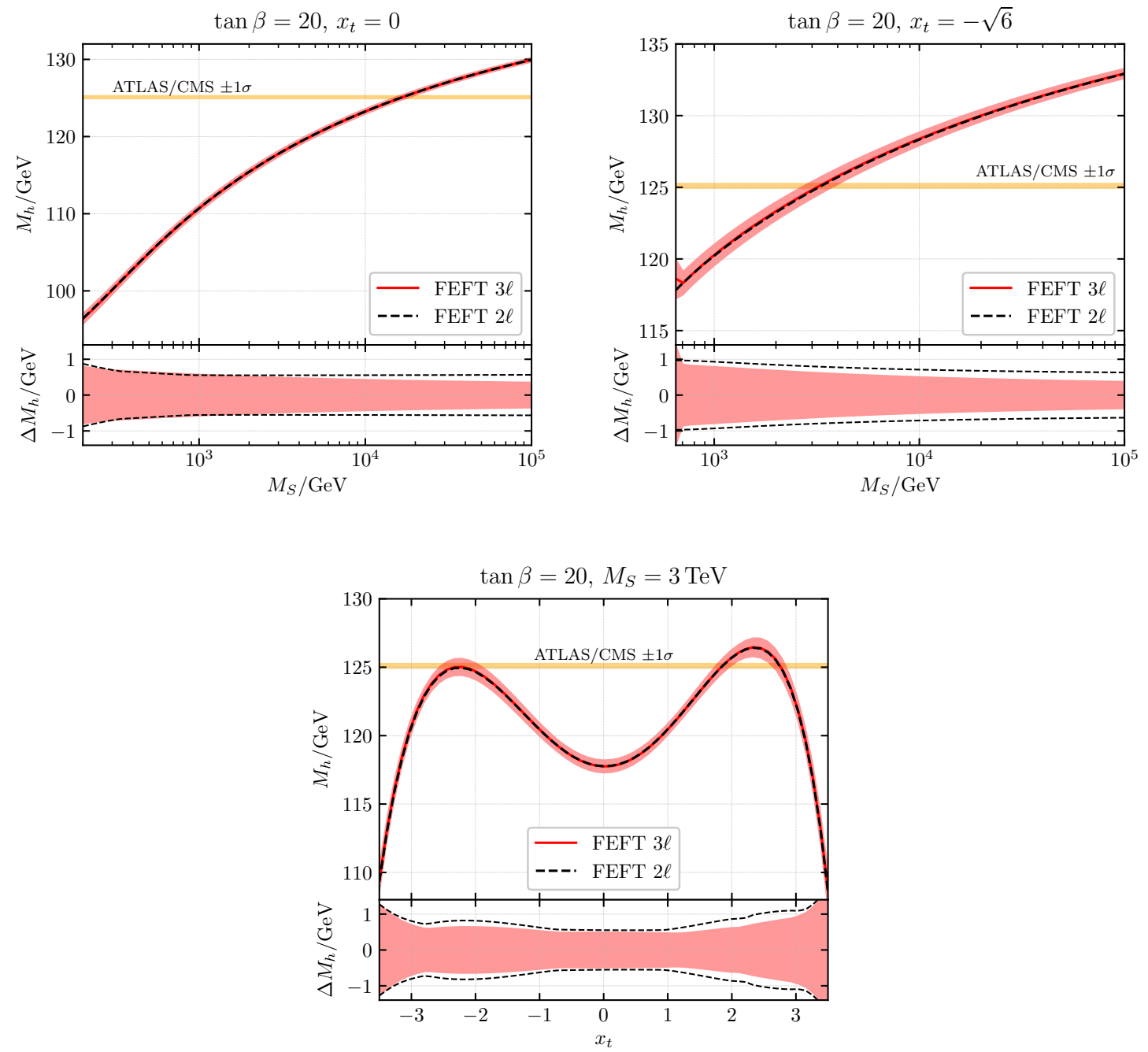

Figure 9. Light CP-even Higgs pole mass predictions with FEFT $2 \ell / 3 \ell$ including the combined uncertainty estimates. The orange band represents the experimentally measured value of the Higgs mass, $M_{h}=(125.10 \pm 0.14) \mathrm{GeV}$, including the experimental uncertainty.

$\left|x_{t}\right| \lesssim 3 .^{23}$ This combined uncertainty becomes smaller for $\left|x_{t}\right| \rightarrow 0$ and larger $M_{S}$, where it can reach $\Delta M_{h} \sim 0.5 \mathrm{GeV}$. These findings are compatible with the uncertainty estimates of refs. [60,61], where hybrid calculations with a comparable precision were studied. For large SUSY scales of $M_{S} \gtrsim 5 \mathrm{TeV}$ we find that the remaining uncertainty of the FEFT $3 \ell$ calculation is dominated by the low-scale uncertainty induced by the determination of the top Yukawa coupling and the electroweak part of the high-scale uncertainty, which can be of similar size.

\footnotetext{
${ }^{23}$ Note, that $\Delta M_{h}$ is a measure of missing higher-order corrections in the relation between the predicted light CP-even Higgs pole mass and the $\overline{\mathrm{DR}}^{\prime}$ input parameters. As was stressed in ref. [61], there are additional uncertainties when the $\overline{\mathrm{DR}}^{\prime}$ input parameters are related to other physical observables.
} 


\section{Conclusions}

We have presented an extension of the FlexibleEFTHiggs method to calculate the SM-like Higgs pole mass in the MSSM. The method combines the virtues of an EFT and fixedorder calculation, resulting in a prediction that includes power-suppressed corrections and a resummation of large logarithms. We have applied our method to perform a state-of-theart calculation of the light CP-even Higgs pole mass in the MSSM, including corrections up to the 3-loop level and resummation of large logarithmic corrections up to $\mathrm{N}^{3} \mathrm{LL}$.

The key of our extension is the generation of a consistent automatized pole mass matching procedure beyond the 1-loop level. The consistency of the FlexibleEFTHiggs method in this regard refers the cancellation of large logarithmic loop corrections and the inclusion of power-suppressed contributions in the matching to the EFT (assumed to be the Standard Model), thereby avoiding problems of double counting. Conceptually, this was achieved by a paradigm shift where the usually applied EFT-parametrized formulation of the high-scale matching was replaced by a parametrization in terms of full-model (MSSM) parameters. Technically, it required the inclusion of derivatives of the SM self energies and tadpoles in the multi-loop matching relations as described in section 4 .

A thorough study of the new full-model parametrization shows that the new approach automatically resums leading contributions in the stop-mixing parameter $x_{t}$, analogously to the well known $\tan \beta$-resummation. This $x_{t}$-resummation leads to significantly stabilized convergence of the perturbation series. For instance, in standard parameter scenarios such as in figure 3 and 4, the numerical impact of the known 2-loop (gaugeless) and 3-loop (leading QCD) threshold corrections is reduced to less than $\sim 0.3 \mathrm{GeV}$, compared to an impact of order $0.5-1.5 \mathrm{GeV}$ in EFT-parametrized calculations.

Next, we have performed a detailed analysis of missing higher-order contributions of our 3-loop FlexibleEFTHiggs calculation. We have employed several different methods of uncertainty estimates, which have a complementary sensitivity to different types of missing higher-order contributions. Our analysis indicates that the remaining theory uncertainty of our calculation is dominated by $(i)$ missing loop corrections to the top Yukawa coupling at the electroweak scale and (ii) missing electroweak 2-loop corrections to the quartic Higgs coupling at the SUSY scale, as shown in figure 8. Numerically, we find that the remaining theory uncertainty of our 3-loop FlexibleEFTHiggs calculation amounts to $\Delta M_{h} \lesssim 1 \mathrm{GeV}$ for SUSY scales above $1 \mathrm{TeV}$ and a stop-mixing of $x_{t} \lesssim 3$. This uncertainty is reduced to $\Delta M_{h} \sim 0.5 \mathrm{GeV}$ for vanishing stop-mixing and/or SUSY scales of $M_{S} \gtrsim 10 \mathrm{TeV}$.

Finally, we note that the resummation effects might be of high relevance for nonminimal supersymmetric extensions of the Standard Model, where the loop corrections to the Higgs mass are not known to the same order as in the MSSM. There, the matching correction in the full-model parametrization at NLO, for example, would result in a resummation of highest stop-mixing contributions of $\mathcal{O}\left(\hat{y}_{t}^{2}\left(\hat{y}_{t}^{2}+\hat{g}_{1,2}^{2}\right) \hat{g}_{3}^{2 n}\right)$ with $n>1$, making resummation effects more advisable. 


\section{Acknowledgments}

We are grateful to Jonas Klappert for helpful communication on the Himalaya library and to Henning Bahl for discussions about details of hybrid calculations. We thank Ulrich Nierste for detailed discussions of $\tan \beta$-resummation and of refs. [75, 76]. This research was supported by the German Research Foundation (DFG) under grant number STO 876/2-2 and by the high-performance computing cluster Taurus at ZIH, TU Dresden.

\section{A Higgs pole mass matching for $\Delta \lambda$ at $\mathcal{O}\left(y_{t}^{6}\right)$}

In this appendix we show how logarithmic contributions cancel against each other in the (implemented) master formula (4.8) at $\mathcal{O}\left(y_{t}^{6}\right)$, yielding a threshold correction in eq. (4.33) which is equivalent to the results presented in the literature. For the sake of brevity we consider the single scale scenario, where all $\overline{\mathrm{DR}}^{\prime}$ SUSY mass parameters and the matching scale are set equal to $M_{S}$, i.e. $m_{\tilde{f}_{3}}^{2}=M_{i}^{2}=\mu^{2}=m_{A}^{2}=Q^{2}=M_{S}^{2}(f=q, u, d, l, e)$. Furthermore, to keep the expressions short, we consider a scenario with a sufficiently large value of $\tan \beta$, such that a power expansion in $\cot \beta \equiv c t_{\beta}$ up to terms $\alpha c t_{\beta}^{2}$ is reasonable. According to eq. (4.28c) the 2-loop threshold correction is obtained as

$$
\left.\Delta \lambda^{2 \ell}\right|_{y_{t}^{6}}=\frac{1}{v^{2}}\left(\Delta s_{h}^{\mathrm{MSSM}, y_{t}^{6}}-\Delta s_{h}^{\mathrm{SM}, y_{t}^{6}}\right)-2 \frac{\Delta v^{\alpha_{t}}}{v^{3}}\left(\Delta s_{h}^{\mathrm{MSSM}, y_{t}^{4}}-\Delta s_{h}^{\mathrm{SM}, y_{t}^{4}}\right) .
$$

At the considered order, the Higgs pole mass correction in the MSSM in the gaugeless limit is given by

$$
\Delta s_{h}^{\mathrm{MSSM}, y_{t}^{6}}=\left(\Delta m_{h, \mathrm{EP}}^{2, \mathrm{MSSM}}\right)^{y_{t}^{6}}+\left(\Delta m_{h, \mathrm{p}}^{2, \mathrm{MSSM}}\right)^{y_{t}^{6}}
$$

where the first term on the r.h.s. of eq. (A.2) represents the MSSM effective potential contribution from ref. [68],

$$
\begin{aligned}
\left(\Delta m_{h, \mathrm{EP}}^{2, \mathrm{MSSM}}\right)^{y_{t}^{6}}= & \frac{y_{t}^{6} v^{2}}{4(4 \pi)^{4}}\left\{-4\left[48 K+9 L_{S t}^{2}+21 L_{S t}+\pi^{2}-12\right]\right. \\
& +(59-96 K) x_{t}^{4}+8(36 K-17) x_{t}^{2}-6 x_{t}^{6} \\
& -c t_{\beta} 2 x_{t}\left[(96 K+19) x_{t}^{4}-16(24 K+5) x_{t}^{2}+36(16 K+3)\right] \\
& +c t_{\beta}^{2}\left[-(96 K+1) x_{t}^{6}+(576 K-105) x_{t}^{4}+4(73-384 K) x_{t}^{2}\right. \\
& \left.\left.+24\left(38 K+\pi^{2}-7\right)+216 L_{S t}+108 L_{S t}^{2}\right]\right\}
\end{aligned}
$$

where $L_{S t} \equiv \log \left(M_{S}^{2} / m_{t}^{2}\right)$. The second term on the r.h.s. of eq. (A.2) originates from the momentum-dependence of the 1-loop Higgs self-energy. It can be regarded as the difference between the pole mass and the mass shift induced by the MSSM effective potential. The 
SM 2-loop contributions at the considered order are given by

$$
\begin{aligned}
\Delta s_{h}^{\mathrm{SM}, y_{t}^{6}}= & \left(\Delta m_{h, \mathrm{EP}}^{2, \mathrm{SM}}\right)^{y_{t}^{6}}+\left(\frac{\partial}{\partial p^{2}} \Delta s^{\mathrm{SM}, \hat{y}_{t}^{4}}(0)\right) \Delta\left(p^{2}\right)^{\alpha_{t}} \\
& +\left(\frac{\partial}{\partial \hat{y}_{t}} \Delta s^{\mathrm{SM}, \hat{y}_{t}^{4}}(0)\right) \Delta y_{t}^{\alpha_{t}}+\left(\frac{\partial}{\partial \hat{v}} \Delta s^{\mathrm{SM}, \hat{y}_{t}^{4}}(0)\right) \Delta v^{\alpha_{t}}, \\
\left(\Delta m_{h, \mathrm{SP}}^{2, \mathrm{SM}}\right)_{t}^{6}= & \frac{3 y_{t}^{6} v^{2}\left(3 c t_{\beta}^{2}-1\right)}{(4 \pi)^{4}}\left[3 L_{S t}^{2}+7 L_{S t}+2+\frac{\pi^{2}}{3}\right],
\end{aligned}
$$

where the first term on the r.h.s. of eq. (A.4) represents the contribution from the SM effective potential [71] and the other terms are the implicit contributions. The combination of the MSSM and SM momentum contributions reads,

$$
\begin{gathered}
\left(\Delta m_{h, \mathrm{p}}^{2, \mathrm{MSSM}}\right)^{y_{t}^{6}}-\left(\frac{\partial}{\partial p^{2}} \Delta s^{\mathrm{SM}, \hat{y}_{t}^{4}}(0)\right) \Delta\left(p^{2}\right)^{\alpha_{t}} \\
=\frac{y_{t}^{6} v^{2}\left(3 c t_{\beta}^{2}-1\right)}{4(4 \pi)^{4}} x_{t}^{2}\left[12 L_{S t}+12 x_{t}^{2}-x_{t}^{4}\right] .
\end{gathered}
$$

This contribution were for example presented in eq. (A.9) of ref. [42] and have been denoted as WFR contributions. The combination of the remaining (implicit) terms reads

$$
\begin{aligned}
& \frac{2 \Delta v^{\alpha_{t}}}{v}\left(\Delta s_{h}^{\mathrm{SM}, y_{t}^{4}}-\Delta s_{h}^{\mathrm{MSSM}, y_{t}^{4}}\right)-\left(\frac{\partial}{\partial \hat{v}} \Delta s^{\mathrm{SM}, \hat{y}_{t}^{4}}(0)\right) \Delta v^{\alpha_{t}}-\left(\frac{\partial}{\partial \hat{y}_{t}} \Delta s^{\mathrm{SM}, \hat{y}_{t}^{4}}(0)\right) \Delta y_{t}^{\alpha_{t}} \\
& =\frac{y_{t}^{6} v^{2}}{4(4 \pi)^{4}}\left[18 c t_{\beta}^{2}\left(2 L_{S t}-1\right)+\left(1-3 c t_{\beta}^{2}\right) x_{t}^{2}\left(12 L_{S t}-12 x_{t}^{2}+x_{t}^{4}\right)\right] .
\end{aligned}
$$

Note, that these (implicit) contributions arise in our calculation due to our choice of the full-model parametrization of $\hat{\lambda}$. Inserting all contributions from above into eq. (A.1), all large logarithms cancel and one obtains

$$
\begin{aligned}
\left.\Delta \lambda^{2 \ell}\right|_{y_{t}^{6}}= & \frac{y_{t}^{6}}{4(4 \pi)^{4}}\left\{-4 x_{t}^{6}+(35-96 K) x_{t}^{4}+8(36 K-17) x_{t}^{2}-192 K+72\right. \\
& +c t_{\beta}\left[-2(96 K+19) x_{t}^{5}+32(24 K+5) x_{t}^{3}-72(16 K+3) x_{t}\right] \\
& +c t_{\beta}^{2}\left[-(96 K+7) x_{t}^{6}+(576 K-33) x_{t}^{4}+4(73-384 K) x_{t}^{2}\right. \\
& \left.\left.+6\left(152 K+2 \pi^{2}-43\right)\right]\right\} .
\end{aligned}
$$

Inserting the numerical value for the constant $K \simeq-0.1953256$ [68], one arrives at the expression in eq. (4.33).

Open Access. This article is distributed under the terms of the Creative Commons Attribution License (CC-BY 4.0), which permits any use, distribution and reproduction in any medium, provided the original author(s) and source are credited.

\section{References}

[1] ATLAS collaboration, Observation of a new particle in the search for the Standard Model Higgs boson with the ATLAS detector at the LHC, Phys. Lett. B 716 (2012) 1 [arXiv: 1207.7214] [INSPIRE]. 
[2] CMS collaboration, Observation of a New Boson at a Mass of $125 \mathrm{GeV}$ with the CMS Experiment at the LHC, Phys. Lett. B 716 (2012) 30 [arXiv:1207.7235] [InSPIRE].

[3] ATLAS and CMS collaborations, Combined Measurement of the Higgs Boson Mass in pp Collisions at $\sqrt{s}=7$ and $8 \mathrm{TeV}$ with the ATLAS and CMS Experiments, Phys. Rev. Lett. 114 (2015) 191803 [arXiv:1503.07589] [INSPIRE].

[4] Particle Data Group collaboration, Review of Particle Physics, Phys. Rev. D 98 (2018) 030001 [INSPIRE].

[5] ATLAS and CMS collaborations, Measurements of the Higgs boson production and decay rates and constraints on its couplings from a combined ATLAS and CMS analysis of the LHC pp collision data at $\sqrt{s}=7$ and $8 \mathrm{TeV}$, JHEP 08 (2016) 045 [arXiv:1606.02266] [INSPIRE].

[6] R. Hempfling and A.H. Hoang, Two loop radiative corrections to the upper limit of the lightest Higgs boson mass in the minimal supersymmetric model, Phys. Lett. B 331 (1994) 99 [hep-ph/9401219] [INSPIRE].

[7] S. Heinemeyer, W. Hollik and G. Weiglein, Precise prediction for the mass of the lightest Higgs boson in the MSSM, Phys. Lett. B 440 (1998) 296 [hep-ph/9807423] [INSPIRE].

[8] S. Heinemeyer, W. Hollik and G. Weiglein, QCD corrections to the masses of the neutral CP-even Higgs bosons in the MSSM, Phys. Rev. D 58 (1998) 091701 [hep-ph/9803277] [INSPIRE].

[9] S. Heinemeyer, W. Hollik and G. Weiglein, The Masses of the neutral CP-even Higgs bosons in the MSSM: Accurate analysis at the two loop level, Eur. Phys. J. C 9 (1999) 343 [hep-ph/9812472] [INSPIRE].

[10] G. Degrassi, P. Slavich and F. Zwirner, On the neutral Higgs boson masses in the MSSM for arbitrary stop mixing, Nucl. Phys. B 611 (2001) 403 [hep-ph/0105096] [INSPIRE].

[11] A. Brignole, G. Degrassi, P. Slavich and F. Zwirner, On the $O\left(a l p h a_{t}^{2}\right)$ two loop corrections to the neutral Higgs boson masses in the MSSM, Nucl. Phys. B 631 (2002) 195 [hep-ph/0112177] [INSPIRE].

[12] S.P. Martin, Two Loop Effective Potential for a General Renormalizable Theory and Softly Broken Supersymmetry, Phys. Rev. D 65 (2002) 116003 [hep-ph/0111209] [INSPIRE].

[13] S.P. Martin, Two Loop Effective Potential for the Minimal Supersymmetric Standard Model, Phys. Rev. D 66 (2002) 096001 [hep-ph/0206136] [INSPIRE].

[14] S.P. Martin, Complete Two Loop Effective Potential Approximation to the Lightest Higgs Scalar Boson Mass in Supersymmetry, Phys. Rev. D 67 (2003) 095012 [hep-ph/0211366] [INSPIRE].

[15] A. Dedes and P. Slavich, Two loop corrections to radiative electroweak symmetry breaking in the MSSM, Nucl. Phys. B 657 (2003) 333 [hep-ph/0212132] [INSPIRE].

[16] A. Brignole, G. Degrassi, P. Slavich and F. Zwirner, On the two loop sbottom corrections to the neutral Higgs boson masses in the MSSM, Nucl. Phys. B 643 (2002) 79 [hep-ph/0206101] [INSPIRE].

[17] A. Dedes, G. Degrassi and P. Slavich, On the two loop Yukawa corrections to the MSSM Higgs boson masses at large tan beta, Nucl. Phys. B 672 (2003) 144 [hep-ph/0305127] [INSPIRE]. 
[18] S.P. Martin, Two loop scalar self energies in a general renormalizable theory at leading order in gauge couplings, Phys. Rev. D 70 (2004) 016005 [hep-ph/0312092] [INSPIRE].

[19] B.C. Allanach, A. Djouadi, J.L. Kneur, W. Porod and P. Slavich, Precise determination of the neutral Higgs boson masses in the MSSM, JHEP 09 (2004) 044 [hep-ph/0406166] [INSPIRE].

[20] S.P. Martin, Strong and Yukawa two-loop contributions to Higgs scalar boson self-energies and pole masses in supersymmetry, Phys. Rev. D 71 (2005) 016012 [hep-ph/0405022] [INSPIRE].

[21] S. Heinemeyer, W. Hollik, H. Rzehak and G. Weiglein, High-precision predictions for the MSSM Higgs sector at $O\left(\alpha_{b} \alpha_{s}\right)$, Eur. Phys. J. C 39 (2005) 465 [hep-ph/0411114] [INSPIRE].

[22] S.P. Martin, Two-loop scalar self-energies and pole masses in a general renormalizable theory with massless gauge bosons, Phys. Rev. D 71 (2005) 116004 [hep-ph/0502168] [INSPIRE].

[23] S.P. Martin, Three-loop corrections to the lightest Higgs scalar boson mass in supersymmetry, Phys. Rev. D 75 (2007) 055005 [hep-ph/0701051] [INSPIRE].

[24] S. Heinemeyer, W. Hollik, H. Rzehak and G. Weiglein, The Higgs sector of the complex MSSM at two-loop order: QCD contributions, Phys. Lett. B 652 (2007) 300 [arXiv: 0705.0746] [INSPIRE].

[25] R.V. Harlander, P. Kant, L. Mihaila and M. Steinhauser, Higgs boson mass in supersymmetry to three loops, Phys. Rev. Lett. 100 (2008) 191602 [arXiv:0803.0672] [INSPIRE].

[26] P. Kant, R.V. Harlander, L. Mihaila and M. Steinhauser, Light MSSM Higgs boson mass to three-loop accuracy, JHEP 08 (2010) 104 [arXiv: 1005.5709] [INSPIRE].

[27] W. Hollik and S. Paßehr, Two-loop top-Yukawa-coupling corrections to the Higgs boson masses in the complex MSSM, Phys. Lett. B 733 (2014) 144 [arXiv:1401.8275] [InSPIRE].

[28] W. Hollik and S. Paßehr, Higgs boson masses and mixings in the complex MSSM with two-loop top-Yukawa-coupling corrections, JHEP 10 (2014) 171 [arXiv:1409.1687] [INSPIRE].

[29] G. Degrassi, S. Di Vita and P. Slavich, Two-loop QCD corrections to the MSSM Higgs masses beyond the effective-potential approximation, Eur. Phys. J. C $\mathbf{7 5}$ (2015) 61 [arXiv: 1410.3432] [INSPIRE].

[30] S. Borowka, T. Hahn, S. Heinemeyer, G. Heinrich and W. Hollik, Momentum-dependent two-loop QCD corrections to the neutral Higgs-boson masses in the MSSM, Eur. Phys. J. C 74 (2014) 2994 [arXiv: 1404.7074] [INSPIRE].

[31] S. Borowka, T. Hahn, S. Heinemeyer, G. Heinrich and W. Hollik, Renormalization scheme dependence of the two-loop QCD corrections to the neutral Higgs-boson masses in the MSSM, Eur. Phys. J. C 75 (2015) 424 [arXiv: 1505.03133] [INSPIRE].

[32] M.D. Goodsell and F. Staub, The Higgs mass in the CP-violating MSSM, NMSSM and beyond, Eur. Phys. J. C 77 (2017) 46 [arXiv:1604.05335] [InSPIRE].

[33] R.V. Harlander, J. Klappert and A. Voigt, Higgs mass prediction in the MSSM at three-loop level in a pure $\overline{D R}$ context, Eur. Phys. J. C 77 (2017) 814 [arXiv: 1708.05720] [InSPIRE].

[34] S. Paßehr and G. Weiglein, Two-loop top and bottom Yukawa corrections to the Higgs-boson masses in the complex MSSM, Eur. Phys. J. C 78 (2018) 222 [arXiv:1705. 07909] [InSPIRE]. 
[35] D. Stöckinger and J. Unger, Three-loop MSSM Higgs-boson mass predictions and regularization by dimensional reduction, Nucl. Phys. B 935 (2018) 1 [arXiv:1804.05619] [INSPIRE].

[36] S. Borowka, S. Paßehr and G. Weiglein, Complete two-loop QCD contributions to the lightest Higgs-boson mass in the MSSM with complex parameters, Eur. Phys. J. C 78 (2018) 576 [arXiv: 1802.09886] [INSPIRE].

[37] A.R. Fazio and E.A. Reyes R., The Lightest Higgs Boson Mass of the MSSM at Three-Loop Accuracy, Nucl. Phys. B 942 (2019) 164 [arXiv:1901.03651] [InSPIRE].

[38] M.D. Goodsell and S. Paßehr, All two-loop scalar self-energies and tadpoles in general renormalisable field theories, Eur. Phys. J. C 80 (2020) 417 [arXiv:1910. 02094] [InSPIRE].

[39] P. Draper and H. Rzehak, A Review of Higgs Mass Calculations in Supersymmetric Models, Phys. Rept. 619 (2016) 1 [arXiv: 1601.01890] [InSPIRE].

[40] P. Draper, G. Lee and C.E.M. Wagner, Precise estimates of the Higgs mass in heavy supersymmetry, Phys. Rev. D 89 (2014) 055023 [arXiv:1312.5743] [INSPIRE].

[41] E. Bagnaschi, G.F. Giudice, P. Slavich and A. Strumia, Higgs Mass and Unnatural Supersymmetry, JHEP 09 (2014) 092 [arXiv:1407.4081] [INSPIRE].

[42] J. Pardo Vega and G. Villadoro, SusyHD: Higgs mass Determination in Supersymmetry, JHEP 07 (2015) 159 [arXiv: 1504.05200] [INSPIRE].

[43] G. Lee and C.E.M. Wagner, Higgs bosons in heavy supersymmetry with an intermediate $m_{A}$, Phys. Rev. D 92 (2015) 075032 [arXiv:1508.00576] [INSPIRE].

[44] E. Bagnaschi, J. Pardo Vega and P. Slavich, Improved determination of the Higgs mass in the MSSM with heavy superpartners, Eur. Phys. J. C 77 (2017) 334 [arXiv:1703.08166] [INSPIRE].

[45] J. Braathen, M.D. Goodsell and P. Slavich, Matching renormalisable couplings: simple schemes and a plot, Eur. Phys. J. C 79 (2019) 669 [arXiv:1810.09388] [INSPIRE].

[46] M. Gabelmann, M. Mühlleitner and F. Staub, Automatised matching between two scalar sectors at the one-loop level, Eur. Phys. J. C 79 (2019) 163 [arXiv:1810.12326] [InSPIRE].

[47] B.C. Allanach and A. Voigt, Uncertainties in the Lightest CP Even Higgs Boson Mass Prediction in the Minimal Supersymmetric Standard Model: Fixed Order Versus Effective Field Theory Prediction, Eur. Phys. J. C 78 (2018) 573 [arXiv: 1804.09410] [InSPIRE].

[48] R.V. Harlander, J. Klappert, A.D. Ochoa Franco and A. Voigt, The light CP-even MSSM Higgs mass resummed to fourth logarithmic order, Eur. Phys. J. C 78 (2018) 874 [arXiv: 1807.03509] [INSPIRE].

[49] E. Bagnaschi, G. Degrassi, S. Paßehr and P. Slavich, Full two-loop QCD corrections to the Higgs mass in the MSSM with heavy superpartners, Eur. Phys. J. C 79 (2019) 910 [arXiv: 1908.01670] [INSPIRE].

[50] M. Krämer, B. Summ and A. Voigt, Completing the scalar and fermionic Universal One-Loop Effective Action, JHEP 01 (2020) 079 [arXiv:1908.04798] [INSPIRE].

[51] H. Bahl, I. Sobolev and G. Weiglein, Precise prediction for the mass of the light MSSM Higgs boson for the case of a heavy gluino, arXiv:1912.10002 [INSPIRE]. 
[52] T. Hahn, S. Heinemeyer, W. Hollik, H. Rzehak and G. Weiglein, High-Precision Predictions for the Light CP -Even Higgs Boson Mass of the Minimal Supersymmetric Standard Model, Phys. Rev. Lett. 112 (2014) 141801 [arXiv:1312.4937] [INSPIRE].

[53] H. Bahl and W. Hollik, Precise prediction for the light MSSM Higgs boson mass combining effective field theory and fixed-order calculations, Eur. Phys. J. C 76 (2016) 499 [arXiv: 1608.01880] [INSPIRE].

[54] P. Athron, J.-h. Park, T. Steudtner, D. Stöckinger and A. Voigt, Precise Higgs mass calculations in (non-)minimal supersymmetry at both high and low scales, JHEP 01 (2017) 079 [arXiv: 1609.00371] [INSPIRE].

[55] F. Staub and W. Porod, Improved predictions for intermediate and heavy Supersymmetry in the MSSM and beyond, Eur. Phys. J. C 77 (2017) 338 [arXiv:1703.03267] [InSPIRE].

[56] P. Athron et al., FlexibleSUSY 2.0: Extensions to investigate the phenomenology of SUSY and non-SUSY models, Comput. Phys. Commun. 230 (2018) 145 [arXiv:1710.03760] [INSPIRE].

[57] H. Bahl, S. Heinemeyer, W. Hollik and G. Weiglein, Reconciling EFT and hybrid calculations of the light MSSM Higgs-boson mass, Eur. Phys. J. C 78 (2018) 57 [arXiv:1706.00346] [INSPIRE].

[58] H. Bahl and W. Hollik, Precise prediction of the MSSM Higgs boson masses for low $M_{A}$, JHEP 07 (2018) 182 [arXiv: 1805.00867] [INSPIRE].

[59] E.A. Reyes R. and A.R. Fazio, Comparison of the EFT Hybrid and Three-Loop Fixed-Order Calculations of the Lightest MSSM Higgs Boson Mass, Phys. Rev. D 100 (2019) 115017 [arXiv: 1908.00693] [INSPIRE].

[60] R.V. Harlander, J. Klappert and A. Voigt, The light CP-even MSSM Higgs mass including $N^{3} L O+N^{3} L L$ QCD corrections, Eur. Phys. J. C 80 (2020) 186 [arXiv:1910.03595] [INSPIRE].

[61] H. Bahl, S. Heinemeyer, W. Hollik and G. Weiglein, Theoretical uncertainties in the MSSM Higgs boson mass calculation, Eur. Phys. J. C 80 (2020) 497 [arXiv:1912.04199] [InSPIRE].

[62] P. Athron, J.-h. Park, D. Stöckinger and A. Voigt, FlexibleSUSY - A spectrum generator generator for supersymmetric models, Comput. Phys. Commun. 190 (2015) 139 [arXiv: 1406.2319] [INSPIRE].

[63] H. Bahl, Pole mass determination in presence of heavy particles, JHEP 02 (2019) 121 [arXiv: 1812.06452] [INSPIRE].

[64] J. Fleischer, F. Jegerlehner, O.V. Tarasov and O.L. Veretin, Two loop QCD corrections of the massive fermion propagator, Nucl. Phys. B 539 (1999) 671 [Erratum ibid. 571 (2000) 511] [hep-ph/9803493] [inSPIRE].

[65] A.V. Bednyakov, Running mass of the b-quark in QCD and SUSY QCD, Int. J. Mod. Phys. A 22 (2007) 5245 [arXiv:0707.0650] [InSPIRE].

[66] A. Bednyakov, A. Onishchenko, V. Velizhanin and O. Veretin, Two loop $O\left(\alpha_{s}^{2}\right)$ MSSM corrections to the pole masses of heavy quarks, Eur. Phys. J. C 29 (2003) 87 [hep-ph/0210258] [INSPIRE].

[67] A. Bednyakov, D.I. Kazakov and A. Sheplyakov, On the two-loop $O\left(\alpha_{s}^{2}\right)$ corrections to the pole mass of the t-quark in the MSSM, Phys. Atom. Nucl. 71 (2008) 343 [hep-ph/0507139] [INSPIRE]. 
[68] J.R. Espinosa and R.-J. Zhang, Complete two loop dominant corrections to the mass of the lightest CP even Higgs boson in the minimal supersymmetric standard model, Nucl. Phys. B 586 (2000) 3 [hep-ph/0003246] [INSPIRE].

[69] G. Degrassi et al., Higgs mass and vacuum stability in the Standard Model at NNLO, JHEP 08 (2012) 098 [arXiv: 1205.6497] [INSPIRE].

[70] S.P. Martin and D.G. Robertson, Higgs boson mass in the Standard Model at two-loop order and beyond, Phys. Rev. D 90 (2014) 073010 [arXiv:1407.4336] [INSPIRE].

[71] C. Ford, I. Jack and D.R.T. Jones, The Standard model effective potential at two loops, Nucl. Phys. B 387 (1992) 373 [Erratum ibid. 504 (1997) ] [hep-ph/0111190] [INSPIRE].

[72] D.M. Pierce, J.A. Bagger, K.T. Matchev and R.-j. Zhang, Precision corrections in the minimal supersymmetric standard model, Nucl. Phys. B 491 (1997) 3 [hep-ph/9606211] [INSPIRE].

[73] L.J. Hall, R. Rattazzi and U. Sarid, The Top quark mass in supersymmetric $\mathrm{SO}(10)$ unification, Phys. Rev. D 50 (1994) 7048 [hep-ph/9306309] [INSPIRE].

[74] M. Carena, M. Olechowski, S. Pokorski and C.E.M. Wagner, Electroweak symmetry breaking and bottom-top Yukawa unification, Nucl. Phys. B 426 (1994) 269 [hep-ph/9402253] [INSPIRE].

[75] M. Carena, D. Garcia, U. Nierste and C.E.M. Wagner, Effective Lagrangian for the $\bar{t} b H^{+}$ interaction in the MSSM and charged Higgs phenomenology, Nucl. Phys. B 577 (2000) 88 [hep-ph/9912516] [INSPIRE].

[76] L. Hofer, U. Nierste and D. Scherer, Resummation of tan-beta-enhanced supersymmetric loop corrections beyond the decoupling limit, JHEP 10 (2009) 081 [arXiv:0907.5408] [INSPIRE].

[77] T. Kwasnitza and D. Stöckinger, Resummation of QCD-enhanced squark-mixing contributions in Higgs mass calculations, in preparation.

[78] L.N. Mihaila, J. Salomon and M. Steinhauser, Gauge Coupling $\beta$-functions in the Standard Model to Three Loops, Phys. Rev. Lett. 108 (2012) 151602 [arXiv:1201.5868] [INSPIRE].

[79] A.V. Bednyakov, A.F. Pikelner and V.N. Velizhanin, Anomalous dimensions of gauge fields and gauge coupling $\beta$-functions in the Standard Model at three loops, JHEP 01 (2013) 017 [arXiv:1210.6873] [INSPIRE].

[80] A.V. Bednyakov, A.F. Pikelner and V.N. Velizhanin, Yukawa coupling $\beta$-functions in the Standard Model at three loops, Phys. Lett. B 722 (2013) 336 [arXiv:1212.6829] [InSPIRE].

[81] K.G. Chetyrkin and M.F. Zoller, Three-loop $\beta$-functions for top-Yukawa and the Higgs self-interaction in the Standard Model, JHEP 06 (2012) 033 [arXiv:1205.2892] [INSPIRE].

[82] A.V. Bednyakov, A.F. Pikelner and V.N. Velizhanin, Higgs self-coupling $\beta$-function in the Standard Model at three loops, Nucl. Phys. B 875 (2013) 552 [arXiv:1303.4364] [InSPIRE].

[83] K.G. Chetyrkin and M.F. Zoller, Leading QCD-induced four-loop contributions to the $\beta$-function of the Higgs self-coupling in the SM and vacuum stability, JHEP 06 (2016) 175 [arXiv: 1604.00853] [INSPIRE].

[84] S.P. Martin, Four-Loop Standard Model Effective Potential at Leading Order in QCD, Phys. Rev. D 92 (2015) 054029 [arXiv: 1508.00912] [INSPIRE].

[85] A.V. Bednyakov and A.F. Pikelner, Four-loop strong coupling $\beta$-function in the Standard Model, Phys. Lett. B 762 (2016) 151 [arXiv:1508.02680] [InSPIRE]. 
[86] I. Jack, D.R.T. Jones and A.F. Kord, Three loop soft running, benchmark points and semiperturbative unification, Phys. Lett. B 579 (2004) 180 [hep-ph/0308231] [INSPIRE].

[87] I. Jack, D.R.T. Jones and A.F. Kord, Snowmass benchmark points and three-loop running, Annals Phys. 316 (2005) 213 [hep-ph/0408128] [INSPIRE].

[88] K.G. Chetyrkin and M. Steinhauser, The Relation between the MS-bar and the on-shell quark mass at order $\alpha_{s}^{3}$, Nucl. Phys. B 573 (2000) 617 [hep-ph/9911434] [INSPIRE].

[89] K. Melnikov and T.v. Ritbergen, The Three loop relation between the MS-bar and the pole quark masses, Phys. Lett. B 482 (2000) 99 [hep-ph/9912391] [INSPIRE].

[90] S.P. Martin, Top-quark pole mass in the tadpole-free $\overline{M S}$ scheme, Phys. Rev. D 93 (2016) 094017 [arXiv: 1604.01134] [INSPIRE].

[91] S. Fanchiotti, B.A. Kniehl and A. Sirlin, Incorporation of QCD effects in basic corrections of the electroweak theory, Phys. Rev. D 48 (1993) 307 [hep-ph/9212285] [INSPIRE].

[92] S. Bethke, The 2009 World Average of $\alpha_{s}$, Eur. Phys. J. C 64 (2009) 689 [arXiv: 0908.1135] [INSPIRE].

[93] Particle Data Group collaboration, Review of Particle Physics, Chin. Phys. C 38 (2014) 090001 [INSPIRE].

[94] Particle Data Group collaboration, Review of Particle Physics (RPP), Phys. Rev. D 86 (2012) 010001 [INSPIRE].

[95] ATLAS, CDF, CMS and D0 collaborations, First combination of Tevatron and LHC measurements of the top-quark mass, arXiv:1403.4427 [INSPIRE].

[96] S.P. Martin and M.T. Vaughn, Two loop renormalization group equations for soft supersymmetry breaking couplings, Phys. Rev. D 50 (1994) 2282 [Erratum ibid. 78 (2008) 039903] [hep-ph/9311340] [INSPIRE].

[97] J.R. Espinosa and R.-J. Zhang, MSSM lightest CP even Higgs boson mass to $O\left(\alpha_{s} \alpha_{t}\right)$ : The Effective potential approach, JHEP 03 (2000) 026 [hep-ph/9912236] [INSPIRE]. 